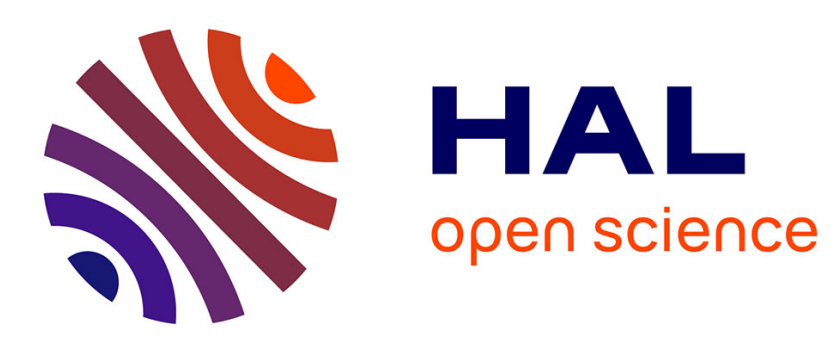

\title{
Anion recognition by phosphonium calix[4] arenes: synthesis and physico-chemical studies
}

\author{
Radoslaw Pomeæko, Zouhair Asfari, Véronique Hubscher-Bruder, Maria
} Bochenska, Françoise Arnaud-Neu

\section{- To cite this version:}

Radoslaw Pomeæko, Zouhair Asfari, Véronique Hubscher-Bruder, Maria Bochenska, Françoise Arnaud-Neu. Anion recognition by phosphonium calix[4]arenes: synthesis and physico-chemical studies. Supramolecular Chemistry, 2010, 22 (05), pp.275-288. 10.1080/10610270903437051 . hal00586992

\section{HAL Id: hal-00586992 \\ https://hal.science/hal-00586992}

Submitted on 19 Apr 2011

HAL is a multi-disciplinary open access archive for the deposit and dissemination of scientific research documents, whether they are published or not. The documents may come from teaching and research institutions in France or abroad, or from public or private research centers.
L'archive ouverte pluridisciplinaire HAL, est destinée au dépôt et à la diffusion de documents scientifiques de niveau recherche, publiés ou non, émanant des établissements d'enseignement et de recherche français ou étrangers, des laboratoires publics ou privés. 


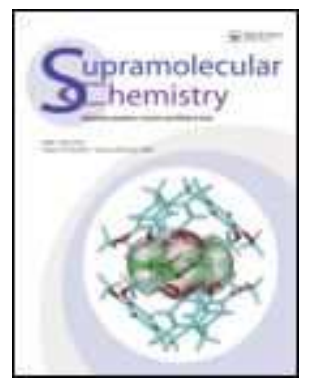

\section{Anion recognition by phosphonium calix[4]arenes: synthesis and physico-chemical studies}

\begin{tabular}{|r|l|}
\hline Journal: & Supramolecular Chemistry \\
\hline Manuscript ID: & GSCH-2009-0077.R3 \\
\hline $\begin{array}{r}\text { Manuscript Type: } \\
\text { Author: }\end{array}$ & Full Paper \\
\hline Complete List of Authors: & $\begin{array}{l}\text { Pomeæko, Radosław; Gdansk University of Technology, Chemical } \\
\text { technology } \\
\text { Asfari, Zouhair; IPHC, CNRS, UDS, DSA } \\
\text { Hubscher-Bruder, Véronique; IPHC, CNRS, UDS, DSA } \\
\text { Bochenska, Maria; Gdansk University, Chemical technology } \\
\text { Arnaud-Neu, Françoise; IPHC, CNRS, UDS, DSA }\end{array}$ \\
\hline Keywords: & $\begin{array}{l}\text { Phosphonium calix[4]arenes, anions, binding properties, } \\
\text { microcalorimetry, ion selective electrodes }\end{array}$ \\
\hline
\end{tabular}

\section{$\diamond$ ScholaroNE" \\ Manuscript Central}




\section{Index abstract}

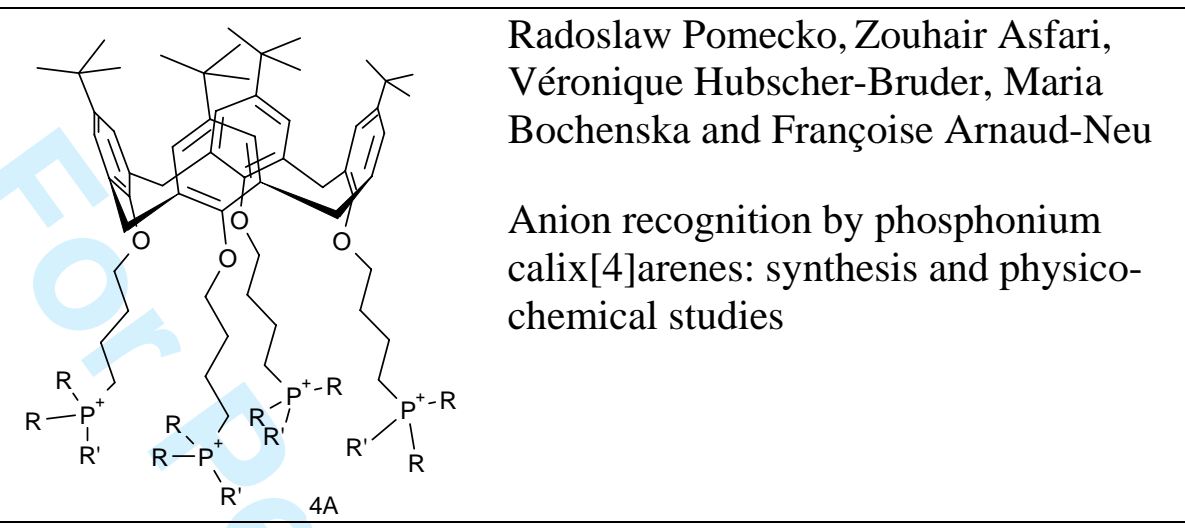




\section{Anion recognition by phosphonium calix[4]arenes : synthesis and physico- chemical studies}

Radoslaw Pomecko, ${ }^{\mathrm{a}, \mathrm{b}}$ Zouhair Asfari, ${ }^{\mathrm{a}}$ Véronique Hubscher-Bruder, ${ }^{\mathrm{a}}$ Maria Bochenska ${ }^{\mathrm{b} *}$ and Françoise Arnaud-Neu ${ }^{\mathrm{a}^{*}}$

${ }^{a}$ IPHC-DSA, ULP, CNRS, ECPM, 25 rue Becquerel, 67087 Strasbourg Cedex 2, France; Email:farnaud@chimie.u-strasbg.fr

${ }^{b}$ Department of Chemical Technology, Chemical Faculty, Gdansk University of Technology, ul. Narutowicza 11/12,80-264 Gdansk, Poland.E-mail: marboch@chem.pg.gda.pl

p-tert-Butylcalix[4]arenes, in the cone conformation, di- and tetrasubstituted at the narrow rim with charged phosphonium groups, have been synthesized and characterized. Their interactions with a wide range of anions have been investigated in solution in chloroform and acetonitrile by means of ${ }^{1} \mathrm{H}$ and ${ }^{31} \mathrm{P}$ NMR and microcalorimetry (ITC). These compounds have also been incorporated as sensing material in PVC ion selective electrodes (ISE). The results showed that they interact strongly with the more lipophilic anions $\mathrm{ClO}_{4}{ }^{-}, \mathrm{SCN}^{-}$and $\mathrm{I}^{-}$, in solution as in the electrode membranes. The origin of this selectivity is discussed and, in particular, the role of the salt counterion is examined.

Keywords: Phosphonium calix[4]arenes; anion binding properties; microcalorimetry; ion selective electrodes.

\section{INTRODUCTION}

Anions play an important role in many biological processes, such as regulation of cell activity, synthesis of proteins, transport of hormones [1,2,3]. They are also frequently used in many industrial technologies, which very often generate an increase of the concentration of anions in the environment or even introduce anionic species which were unknown so far in ecosystems. The presence of these anions is crucial in environmental and medical concerns, as they are pollutants and may have harmful effects on living organisms and human health $[4,5,6]$. Therefore there is a need of fast and selective anion detection methods allowing real-time monitoring of anion concentration changes and of efficient clean up processes. Design of anion receptors for such applications remains a great challenge for chemists because they have to take into consideration the specific 
anion properties, such as a large range of shapes and geometries, small electric charges $v s$. sizes, high free energies of solvation, and in some cases multiple oxidation states of the central atoms in oxoanions or $\mathrm{pH}$ dependence. In many artificial anion hosts, noncovalent interactions are responsible for host-guest recognition. They include electrostatic interactions, hydrogen bonding, hydrophobic effects, coordination to a metal ion or combinations of these interactions. The hosts can be neutral, containing urea $[7,8,9,10]$, thiourea $[11,12]$ or amide functions [13]. They can also be positively charged, containing pyridinium [14], polyammonium [15] or quaternary ammonium [16] binding sites. Calix[4]arenes [17,18] and porphyrins [19] are often used as scaffolds onto which these functional groups can be grafted. Calixpyrroles are also known as efficient anion receptors $[20,21]$.

Recently, we described the synthesis and characterization of a new calix[4]arene derivative (5) bearing four positively charged triphenyl phosphonium groups [22]. The presence of these highly polarisable moieties, where the charge is spread over the three aromatic rings, was expected to favour the interaction with lipophilic anions. Preliminary binding studies showed that this ligand interacted selectively with some anions, namely $\mathrm{ClO}_{4}{ }^{-}$and $\mathrm{SCN}^{-}$. This compound was also incorporated, as ionophore-sensing material, in ion selective electrodes (ISEs) which exhibited a selectivity order similar to the Hofmeister series. The disubstituted derivative 1 was also synthesised as the hexafluorophosphate [23].

In order to get more information on the mechanisms involved in the recognition process and to optimize its selectivity, we have now extended this study to new di- and tetrasubstituted phosphonium calix[4]arene derivatives (compounds $\mathbf{2 - 4 , 6}$ and 7) and reexamined the properties of 1 and 5 (Fig. 1). In some of these compounds, one of the phenyl rings on the phosphonium groups has been replaced by a methyl radical or a hydrogen atom. The presence of such small substituents is expected to increase the charge density on the phosphorus atoms and their accessibility [24]. Moreover, the presence of hydrogen atoms may induce the formation of hydrogen bonds with anions. The possibility of tuning the charge density on the phosphorus atoms by changing the nature of the their substituents could allow the design of receptors able to distinguish lipophilic anions like $\mathrm{ClO}_{4}^{-}, \mathrm{SCN}^{-}, \mathrm{I}^{-}$or $\mathrm{NO}_{3}{ }^{-}$from other anions and between them. The binding properties of these compounds towards a variety of anions have been followed by ${ }^{1} \mathrm{H}$ and ${ }^{31} \mathrm{P}$ NMR and by titration microcalorimetry (ITC). In particular the role of the salt counterion was examined using these techniques. 


\section{Fig. 1}

\section{RESULTS AND DISCUSSION}

\section{Synthesis}

The ligands synthesised in this work (Fig. 1) are based on a tetrakis-p-tertbutylcalix[4]arene platform which presents several advantages. It has a well defined size and is readily amenable to substitution at its lower rim where ligating groups can be attached and in some extent preorganized. The amphiphilicity of such derivatives should allow their introduction into the membranes of ion selective electrodes.

Diphosphonium ligands were synthesised in two or three steps according to the known procedure [23]. The first step was the selective bromoalkylation of the tetrakis- $p$-tertbutylcalix[4]arene $\left(\mathbf{S}_{\mathbf{1}}\right)$, leading to the intermediate molecules $\mathbf{S}_{\mathbf{2}}$ or $\mathbf{S}_{\mathbf{3}}$ in $71 \%$ and $55 \%$ yield, respectively (Scheme 1). 1,3-bis-(4-triphenylphosphonium-butoxy)-p-tert-butylcalix[4]arene dibromide (1) was obtained by the reaction of $\mathbf{S}_{\mathbf{2}}$ with 10 equivalents of triphenylphosphine. After 6 days under reflux in chloroform, the product was precipitated from a dichloromethane/hexane mixture in $76 \%$ yield. 1,3-bis-(4-(P,P-diphenyl-Pmethylphosphonium)-butoxy)-p-tert-butylcalix[4]arene dibromide (2) was obtained during the reaction of $\mathbf{S}_{\mathbf{2}}$ with 10 equivalents of diphenylmethylphosphine in the same conditions in $69 \%$ yield.

\section{Scheme 1}

The more lipophilic molecules $\mathbf{3}$ and $\mathbf{4}$, where the free phenolic protons are substituted with propyl groups, were prepared in order to increase their stability in the lipophilic membrane of ion selective electrodes. The reaction of $\mathbf{S}_{\mathbf{3}}$ with 10 equivalents of 1,4dibromobutyl, refluxed for 4 days in dimethylformamide in the presence of 7 equivalents of $\mathrm{NaH}$ gave the 1,3-bis-(propoxy)-2,4-bis-(butoxy-4-bromide)-tetrakis-p-tert-butylcalix[4]arene $\left(\mathbf{S}_{\mathbf{4}}\right)$ in $57 \%$ yield. Compounds $\mathbf{3}$ and $\mathbf{4}$ were obtained by the reaction of $\mathbf{S}_{\mathbf{4}}$ with 10 equivalents of triphenylphosphine and 10 equivalents of diphenylmethylphosphine in chloroform, in $76 \%$ and $64 \%$ yield, respectively (Scheme 1).

Tetraphosphonium ligands $\mathbf{6}$ and $\mathbf{7}$ were synthesised according to the procedure already described for 5 [22] from the intermediate molecule tetrakis-(butoxy-4-bromide)-p-tertbutylcalix[4]arene $\left(\mathbf{S}_{\mathbf{5}}\right)$ by substitution of the bromine atoms of the alkyl chains (Scheme 2). The tetrakis-(4-(P,P-diphenyl-P-methylphosphonium)-butoxy)-p-tert-butylcalix[4]arene tetrabromide (6) was obtained from the reaction of $\mathbf{S}_{\mathbf{5}}$ with 20 equivalents of diphenylmethylphosphine in chloroform with $59 \%$ yield. 
Scheme 2

Ligand 7 was synthesised in three steps. The reaction of $\mathbf{S}_{\mathbf{5}}$ with 5 equivalents of $\mathrm{KPPh}_{2}$ gave the product $\mathbf{S}_{\mathbf{6}}$ in $40 \%$ yield [25,26,27]. $\mathbf{S}_{\mathbf{6}}$ was then protonated with an excess of $\mathrm{HBr}$ giving ligand 7 in $52 \%$ yield.

In order to study the influence of the ligand counterion on the ligand - anion interactions, the tetra substituted phosphonium ligands were also synthesised as perchlorates (5a, 6a) and hexafluorophosphates $(\mathbf{5 b}, \mathbf{6 b})$. These compounds were obtained by reacting ligands 5 and $\mathbf{6}$ with the appropriate silver salts.

For comparison purpose, the monomeric subunit of ligand $\mathbf{5}$, the triphenylphosphoniumbutoxy-p-tert-butylphenol bromide (8) was synthesized as previously described [22].

The cone conformation of the di-substituted calix[4]arenes was indicated by the presence in their ${ }^{1} \mathrm{H}$ NMR spectra of two singlets for the tert-butyl protons (at 0.99 and 1.28 ppm for 1 and 0.96 and $1.29 \mathrm{ppm}$ for 2 ) and a $\mathrm{AB}$ system for the methylene protons (at 3.19 and $3.90 \mathrm{ppm}$ for $\mathbf{1}$ and at 3.23 and $4.02 \mathrm{ppm}$ for 2 ). The ${ }^{1} \mathrm{H}$ NMR spectra of ligands $\mathbf{5}$ and $\mathbf{6}$ are characteristic of tetrasubstituted derivatives of calix[4]arenes in the cone conformation. For instance in the case of $\mathbf{6}$, it is indicated by the presence of the singlet corresponding to the protons of the tert-butyl groups at $1.03 \mathrm{ppm}$, the $\mathrm{AB}$ system of the methylene protons of the calixarene at 4.26 and $3.03 \mathrm{ppm}$. Two well defined multiplets for the protons of the aromatic phosphonium groups can be observed as well as one doublet for the methyl protons in direct neighbourhood of the phosphorus atoms.

The spectrum of the protonated phosphonium ligand 7 presents several broad peaks corresponding to multiplets in coalescence. Only two singlets could be clearly observed, one for the tert-butyl groups and one for the aromatic protons of the calix[4]arene, suggesting also the cone conformation of this molecule.

\section{Binding studies \\ ${ }^{1} \mathrm{H}$ and ${ }^{31} \mathrm{P}$ NMR studies in chloroform}

The interactions between the phosphonium calixarenes and the following anions: $\mathrm{NO}_{3}{ }^{-}$, $\mathrm{ClO}_{4}^{-}, \mathrm{I}^{-}, \mathrm{SCN}^{-}, \mathrm{SO}_{4}{ }^{2-}, \mathrm{HCO}_{3}^{-}, \mathrm{Cr}_{2} \mathrm{O}_{7}{ }^{2-}$ provided as sodium salts were studied by ${ }^{1} \mathrm{H}$ and ${ }^{31} \mathrm{P} \mathrm{NMR}$ in $\mathrm{CDCl}_{3}$. Among these anions, only perchlorate, thiocyanate and iodide salts induced changes in the ${ }^{1} \mathrm{H}$ NMR spectra of the ligands. The changes observed in the case of the disubstituted ligand $\mathbf{1}$ upon addition of sodium perchlorate are illustrated in Fig. 2. The main signals affected were those of the $\mathrm{CH}_{2}$ protons $(\mathrm{i}, \mathrm{j})$ directly bound to the carbon 
atoms next to the phosphorus atoms and the aromatic protons $(\mathrm{k})$ of the phosphonium groups (see Table S1, Supplementary Online Material). The shifts of these signals due to changes in the charge density on the phosphorus atoms reflect interactions with these anions.

\section{Figure 2}

Similar but smaller changes were induced in the spectrum of ligand $\mathbf{2}$ by the presence of the same anions (see Table S2, Supplementary Online Material). As with ligand 1, no change is observed for the aromatic protons (c, d) and for the protons (e, f) of the methylene bridge, indicating that the conformation of the calixarene unit is not disturbed. This may be explained by the high rigidity of these disubstituted derivatives due to hydrogen bonds involving the free phenolic groups.

In the ${ }^{31} \mathrm{P}$ NMR spectra of the free ligands $\mathbf{1}$ and $\mathbf{2}$, and of these ligands in the presence of sodium iodide, thiocyanate and perchlorate, the phosphorus atoms appear as a singlet indicating that the two phosphonium groups are chemically equivalent and participate in the anion-ligand interaction. The most important changes in chemical shifts are observed for perchlorate: $\Delta \delta=0.602$ and $0.620 \mathrm{ppm}$ with $\mathbf{1}$ and $\mathbf{2}$, respectively (Table 1).

Table 1

With the tetrasubstituted calix[4]arene 5 previously studied [22] and $\mathrm{ClO}_{4}^{-}, \mathrm{SCN}^{-}$and $\mathrm{I}^{-}$ the most important changes of chemical shifts corresponded to the signals of the methylene bridge protons and the aromatic protons of the calixarene, indicating changes in the conformational tensions of the calixarene scaffold. The signals corresponding to protons of the butyl chains were also shifted as well as the aromatic protons of the phosphonium. On the contrary no change was observed with the monomer 8 [22].

The spectrum of ligand $\mathbf{6}$ was also modified in the presence of these anions (see Table S3, Supplementary Online Material). In particular the signals corresponding to the protons of the calix[4]arene scaffold are moved in a comparable way for the three anions. The changes observed in the spectrum of this ligand in the presence of $\mathrm{ClO}_{4}{ }^{-}$are illustrated in Fig. 3.

Figure 3

With this anion, the multiplet corresponding to the protons (g) and (j) from 3.87 to $3.70 \mathrm{ppm}$ gives two multiplets from 4.36 to $4.17 \mathrm{ppm}$ for (g) and from 3.28 to $3.11 \mathrm{ppm}$ for (j). The multiplet from 8.17 to $8.02 \mathrm{ppm}$ for protons (k) is shifted upfield and gives one multiplet from 7.90 to $7.70 \mathrm{ppm}$, whereas the multiplet from 7.78 to $7.54 \mathrm{ppm}$ of protons $(1, \mathrm{~m})$ does not move significantly. The doublet of the $\mathrm{CH}_{3}$ protons (o) adjacent to the phosphorus atoms at $2.91 \mathrm{ppm}$ is strongly shifted to $2.50 \mathrm{ppm}$. 
The addition of the anions studied to 7 did not induce any change in its ${ }^{1} \mathrm{H}$ NMR spectrum. Especially the signals of the protons of the phosphonium moieties, expected to be involved in hydrogen bond formation, were not shifted.

With ligand $\mathbf{6}$ as with $\mathbf{5}$, only one singlet for the phosphorus atoms was detected in its ${ }^{31} \mathrm{P}$ NMR spectrum indicating all the four phosphonium groups being chemically equivalent. In the presence of sodium perchlorate and thiocyanate, the changes in chemical shifts are larger than those observed with the disubstituted derivatives and suggest stronger interactions (Table 1). With all ligands the most important values were observed for perchlorate.

\section{${ }^{1} \mathrm{H}$ NMR studies in acetonitrile}

${ }^{1} \mathrm{H}$ NMR experiments were repeated with 5 and sodium perchlorate in deuterated acetonitrile, a more dissociating solvent, in which association phenomena are not as important as in chloroform [22]. The spectra of the ligand are very similar in both solvents. In acetonitrile the addition of $\mathrm{NaClO}_{4}$ induces shifts of the same signals as in chloroform. Moreover this study showed the influence of the counterion of the salts on the shifts observed in the spectra (See Table S4, Supplementary Online Material). The signal of the methylene protons ( $\mathrm{g}$ ) adjacent to the phenolic oxygen atoms was shifted only with $\mathrm{NaClO}_{4}$ and $\mathrm{LiClO}_{4}$. With both salts the shifts corresponding to the signals of the protons (j) next to the phosphorus atoms (which are supposed to interact with perchlorate) were similar, whereas with $\mathrm{Et}_{4} \mathrm{NClO}_{4}$ and especially with $\mathrm{CsClO}_{4}$, the values were very small. The changes observed suggested that ligand - anion interaction was connected with the nature of the counterion and its affinity for the ligand.

In order to observe the influence of the ligand counterion, bromide anions were replaced by the more lipophilic perchlorate $(\mathbf{5 a}$ and $\mathbf{6 a})$ or hexafluorophosphate $(\mathbf{5 b}$ and 6b) anions. The chemical shifts $(\delta)$ of selected protons, given in Table 2, show only slight differences for protons (c, e, f) and (g) close to the calix[4]arene scaffold ( $\Delta \delta$ in the range $0.02-0.07 \mathrm{ppm})$. In contrast, the signals of the protons $(\mathrm{j})$ next to the charged phosphorus atoms are greatly shifted $(\Delta \delta=-0.64 \mathrm{ppm}$ for $\mathbf{5 a},-0.75 \mathrm{ppm}$ for $\mathbf{5 b}$ and $\Delta \delta=-0.73 \mathrm{ppm}$ for $\mathbf{6 a},-0.83 \mathrm{ppm}$ for $\mathbf{6 b}$ ). With ligands $\mathbf{6 a}$ and $\mathbf{6 b}$, the signals of the protons (o) of the methyl substituents are also displaced $(\Delta \delta=-0.41 \mathrm{ppm}$ for $\mathbf{6 a}$ and $-0.46 \mathrm{ppm}$ for $\mathbf{6 b})$. These results show that the chemical shifts of the protons next to the phosphonium groups are influenced by the ligand counterion.

Table 2 
On the other hand, it was also shown that when the lipophilic $\mathrm{PF}_{6}^{-}$anions were replacing the $\mathrm{Br}^{-}$counterions of the ligand, there was still a significant shift of the protons (j) close to the phosphorus atoms for ligand $\mathbf{5 b}$ in the presence of $\mathrm{ClO}_{4}{ }^{-}$(Table S4) [22]. This result suggested complexation of perchlorate with this ligand, where no exchange is normally possible.

\section{Microcalorimetric studies in acetonitrile}

In order to get more information on the influence of the counterion of the salt, microcalorimetric titrations were carried out with ligands $\mathbf{5}$ and $\mathbf{5 b}$ against $\mathrm{NaClO}_{4}$, $\mathrm{LiClO}_{4}$ and $\mathrm{Et}_{4} \mathrm{NClO}_{4}$ in acetonitrile. The thermograms recorded during the titration of these ligands with $\mathrm{NaClO}_{4}$ and $\mathrm{LiClO}_{4}$ showed significant exothermic heat effects, whereas their titration with $\mathrm{Et}_{4} \mathrm{NClO}_{4}$ led to no thermal effect (see Figure $\mathrm{S} 1$, Supplementary Online Material). For comparison purpose, the titration of the monomer 8 with $\mathrm{NaClO}_{4}$ was also carried out showing no significant heat effect.

If only the $\mathrm{ClO}_{4}{ }^{-}$anion were involved in the complexation, a similar heat effect should be observed in all the titrations. The fact that an effect is only observed for $\mathrm{NaClO}_{4}$ and $\mathrm{LiClO}_{4}$ suggests that it is not only related to the anion interaction (complexation or anion exchange). Assuming that the large tetraethylammonium cation cannot be complexed with a calix[4] arene, the heat effects observed during the titration with $\mathrm{NaClO}_{4}$ and $\mathrm{LiClO}_{4}$ would rather be due to the complexation of the cations with the two ligands. This is supported by the fact that no heat effect was detected with the monomer 8 , supposed to be unable to complex these cations.

Calorimetric data obtained with $\mathrm{NaClO}_{4}$ and $\mathrm{LiClO}_{4}$ were interpreted assuming different cation complexation models. With sodium and both ligands, the best fit was obtained by considering the presence of $\mathrm{ML}$ and $\mathrm{ML}_{2}$ complexes. The same species were found with $\mathrm{LiClO}_{4}$ and $\mathbf{5 b}$, whereas only a 1:1 complex was formed with $\mathbf{5}$. The formation of $\mathrm{ML}_{2}$ species could be explained by the complexation of the ion pair $\mathrm{Na}^{+} \mathrm{ClO}_{4}{ }^{-}$by two ligands. The stability constants of these complexes are given in Table S5 (see Supplementary Online Material).

An important heat effect was observed during the titration of ligands $\mathbf{5}$ and $\mathbf{5 b}$ against $\mathrm{LiBr}$, which should be related directly to cation complexation as no anion exchange is possible with these ligands. The data interpretation led to species of the same stoichiometry as with $\mathrm{LiClO}_{4}$ (Table S5). The values of the stability constants of 1:1 species formed in the presence of $\mathrm{LiClO}_{4}$ and $\mathrm{LiBr}$ are comparable (with $\mathbf{5}, \log \beta=3.24$ 


\section{Potentiometric studies}

Only few ligands containing phosphorus atoms have been studied so far as active material in ion selective membrane electrodes $[29,30,31]$. They showed a selective response for $\mathrm{ClO}_{4}{ }^{-}$, with, however, little discrimination with respect to $\mathrm{I}^{-}$and $\mathrm{SCN}^{-}$. The results suggested a particular affinity of ligands containing phosphorus atoms for $\mathrm{ClO}_{4}^{-}, \mathrm{SCN}^{-}$and $\mathrm{I}^{-}$anions. By attaching phosphonium moieties to a calix[4]arene scaffold and taking advantage of the preorganization of the ligand, it was expected to enhance the selectivity for tetrahedral or spherical anions. Such selectivity (especially for $\mathrm{ClO}_{4}{ }^{-}$over $\mathrm{I}^{-}$) is hard to obtain with other kinds of receptors.

Disubstituted phosphonium ligands $\mathbf{1}$ and $\mathbf{2}$ were tested as ionophores in the membrane electrodes. The electrodes were sensitive to perchlorate, thiocyanate, iodide and nitrate, showing fast, near-Nernstian responses (Figure 4 and Table S6 of Supplementary Online Material). However, their characteristics changed with time. Attempts to optimize the composition of the conditioning solutions as well as the conditioning time did not improve the situation which might be due to slow leakage of the ionophores from the membrane. These ligands had also the tendency to crystallize in the membrane phase. Crystallization of our ligands within the membranes depends strongly on the kind of plasticizer used. The ligands in the membranes based on bis-(2ethylhexyl)sebacate (BEHS) has crystallized strongly, which decreased their stability. This phenomenon originates from the higher lipophilicity of this plasticizer $(\log P=10.1)$ as compared to 2-nitrophenyloctylether $(o$-NPOE) $(\log P=5.9)$ [32], which is more suitable for charged ligands. Electrodes with membranes based on o-NPOE had the best lifetime and response characteristics and were chosen for further studies. 


\section{Figure 4}

The more lipophilic ligands $\mathbf{3}$ and $\mathbf{4}$, in which n-propyl chains replace the two phenolic $\mathrm{OH}$ groups, were also synthesised. The lifetime of electrodes incorporating these ligands for perchlorate was increased to at least three weeks. The repeatability of the measurements was also good, but their detection limits increased (see Table S7, Supplementary Online Material).

The membranes of electrodes incorporating the tetrasubstituted ligand 6 showed rather quick (within 15-20 s), stable and fully reversible responses (Figure 5 and Table S8 of Supplementary Online Material). The repeatability of the measurements was also good and their lifetime was more than three weeks. They showed close to Nernstian response to $\mathrm{ClO}_{4}{ }^{-}, \mathrm{I}^{-}, \mathrm{NO}_{3}{ }^{-}$and $\mathrm{SCN}^{-}$and no significant response for $\mathrm{SO}_{4}{ }^{2-}, \mathrm{CO}_{3}{ }^{2-}, \mathrm{HPO}_{4}{ }^{2-}, \mathrm{PO}_{4}{ }^{3-}$. A similar behaviour was already observed with electrodes incorporating ligand 5 (Table S8). The highest selectivity was obtained for $\mathrm{ClO}_{4}{ }^{-}$ions in buffered solution $(\mathrm{pH}=5.5)$ and in water $(\mathrm{pH}=6.5)$. The over-Nernstian slope of the electrode response for $\mathrm{Cr}_{2} \mathrm{O}_{7}{ }^{2-}$ could indicate a mechanism where both processes, anion complexation and anion exchange, play an important role. It can also be explained as the presence in the sample of different forms of chromates.

\section{Figure 5}

While the addition of lipophilic anionic sites (KTCIPB) to the membranes in the case of ligand 5 did not change much the properties of the electrodes, it affected the properties of the electrodes containing 6 (Table 3). Without salt, the slope of the electrode is $-54.2 \mathrm{mV}$, and slightly decreases to -51.8 and $-49.9 \mathrm{mV}$, respectively, in the presence of salt. According to the literature data [29,31], such results suggest that none of the ligands works as a neutral carrier because the addition of the lipophilic anion to the membrane does not induce a cationic response of the potentiometric cell. Ligand $\mathbf{6}$ seems to work in the membrane as a typical anion-exchanger, whereas ligand $\mathbf{5}$ could be considered as a charged ligand despite the small influence of KTCIPB.

\section{Table 3}

The influence of the ligand counterions $\left(\mathrm{Br}^{-}, \mathrm{ClO}_{4}{ }^{-}, \mathrm{PF}_{6}{ }^{-}\right)$on the properties of the membrane electrodes was also studied. Table 4 compares the responses to perchlorate of electrodes based on ligands 5a, 6a (perchlorates) and on ligands $\mathbf{5 b}$ and $\mathbf{6 b}$ (hexafluorophosphates) to corresponding electrodes based on ligands 5 and $\mathbf{6}$ (bromides). The less good slope of the electrodes containing $\mathbf{6 a}$ and $\mathbf{6 b}$ as compared to that of electrodes containing 6 suggests rather the anion-exchange nature of the latter ligand. In 
this case the presence of more lipophilic anions (perchlorate or hexafluorophosphate) slows down the anion exchange process. In contrast, the properties of electrodes with the bromide ligand $\mathbf{5}$ and with the perchlorate ligand $\mathbf{5 a}$ are comparable. The presence of highly lipophilic perchlorate anions does not disturb the electrode response, while the presence of hexafluorophosphate anions in the membrane phase (electrode containing ligand $\mathbf{5 b}$ ) decreases the slope and the linearity range.

\section{Table 4}

The poorer properties of the electrode containing $\mathbf{5 b}$ and $\mathbf{6 b}$ could be explained by the higher lipophilicity of hexafluorophosphate anions which hinders the process of anion exchange. The complexation of $\mathrm{ClO}_{4}{ }^{-}$by ligand 5 could explain the good response of electrodes based on $\mathbf{5}$ and $\mathbf{5 a}$ to perchlorate. Such interpretation is consistent with the results of previous experiments (Table 4) and indicates that ligand 5 behaves more like a charged carrier for $\mathrm{ClO}_{4}^{-}$, while ligand 6 behaves more as an anion-exchanger.

The order of selectivity observed with all phosphonium ligands 1-6 and 8 follows the Hofmeister series:

$$
\mathrm{ClO}_{4}{ }^{-}>\mathrm{SCN}^{-}>\mathrm{I}^{-}>\mathrm{Cr}_{2} \mathrm{O}_{7}{ }^{2-}>\mathrm{NO}_{3}{ }^{-}>\mathrm{Br}^{-}>\mathrm{HCO}_{3}{ }^{-}>\mathrm{HPO}_{4}{ }^{2-}>\mathrm{SO}_{4}{ }^{2-}
$$

The highest selectivity is observed for perchlorate (Table 5).

\section{Table 5}

The electrodes based on tetraphosphonium derivatives show higher selectivities for perchlorate over thiocyanate, iodide and nitrate than those based on their di-phosphonium counterparts. These selectivities are also better than the selectivity of electrodes based on the monomer $\mathbf{8}$.

The replacement of one phenyl substituent on the phosphorus atoms by one methyl group does not change significantly the selectivity pattern and the values of the selectivity coefficients of electrodes containing either di- or tetraphosphonium ligands. The only exception is the selectivity of electrodes based on compound 5 against $\mathrm{Cr}_{2} \mathrm{O}_{7}{ }^{2-}$ which increases from 2.6 to $3.2 \log$ units.

Electrodes based on alkylated compounds $\mathbf{3}$ and $\mathbf{4}$ are also selective for perchlorate but the selectivity over iodide and thiocyanate is decreased (Table 5).

Ligands 5 and 6 display better potentiometric properties than the protonated cyclam [33] or its copper complex [34] and than a phosphodithia macrocycle [35]. For instance the detection limit is $2.5 \times 10^{-7} \mathrm{M}$ with ligand 5 when compared to $4.2 \times 10^{-6} \mathrm{M}$ for cyclam and $8 \times 10^{-7} \mathrm{M}$ for the phosphodithiamacrocycle. Ligands $\mathbf{6}$ as $\mathbf{5}$ presents generally higher selectivities than TDMACl [36], the protonated cyclam and $[\mathrm{Cu}(\mathrm{cyclam})]^{2+}$. 


\section{CONCLUDING REMARKS}

The different techniques used to assess the binding properties of phosphonium derivatives showed strong interactions with $\mathrm{SCN}^{-}, \mathrm{I}^{-}$and especially $\mathrm{ClO}_{4}{ }^{-}$and pointed out the important role played by the salt counterion $\left(\mathrm{Na}^{+}\right.$or $\left.\mathrm{Li}^{+}\right)$, which may be complexed by the calix[4]arene. Incorporated in PVC membrane electrodes, these molecules are efficient sensing material for anions with a selectivity order following the Hofmeister series generally observed for ion exchangers. However, the electrodes based on tetraphosphonium derivatives showed better selectivities than those based on the diphosphonium analogues or on the monomeric unit, indicating the importance of the ligand preorganisation which should not be observed in the case of simple anionexchangers.

A question which must be addressed concerns the nature of the interaction between the ligands and the anions, e.g. $\mathrm{ClO}_{4}^{-}$. Is it a simple ion-exchange between the bromides of the ligand and this more lipophilic anion, or is it complexation within the charged phosphonium groups? What is the exact role of the salt counterion?

If NMR gives some indications on the changes in the molecule, suggesting interactions, it does not tell if there is complexation or anion exchange, since the nature of the counterion of the ligand has been shown to influence the chemical shifts of the protons near the charged atoms. In favour of ion-exchange is the fact that the most important shifts are observed with the more lipophilic anions $\mathrm{ClO}_{4}^{-}, \mathrm{SCN}^{-}$and $\mathrm{I}^{-}$. The behaviour in selective electrodes is consistent also with this assumption. However, the fact that no change occurred in the spectrum of the monomer, where only exchange is possible, is against this hypothesis. In favour of complexation is the fact that, in the presence of $\mathrm{NaClO}_{4}$, strong shifts are observed for the signal of the protons next to the phosphonium groups in the spectrum of the hexafluorophosphate ligand where no exchange is possible.

On the other hand ${ }^{1} \mathrm{H}$ NMR and microcalorimetry emphasized the importance of the cation which can be complexed in the cavity of the calixarene. With perchlorate, the best interaction takes place with $\mathrm{Na}^{+}$and $\mathrm{Li}^{+}$, whereas little or no interaction occurs with the larger $\mathrm{Et}_{4} \mathrm{~N}^{+}$and $\mathrm{Cs}^{+}$. It can be noted also that the anion plays also a role in the complexation of the cation, since the spectrum of the calixarene part is not affected in the presence of $\mathrm{NO}_{3}{ }^{-}, \mathrm{SO}_{4}{ }^{2-} \mathrm{HCO}_{3}{ }^{-}$and $\mathrm{Cr}_{2} \mathrm{O}_{7}{ }^{2-}$, i.e. the less lipophilic ones.

\section{EXPERIMENTAL}




\section{Synthesis of intermediate compounds}

\section{1,3-bis-(butoxy-4-bromide)-p-tert-butylcalix[4]arene $\left(S_{2}\right)$}

Into a $250 \mathrm{~cm}^{3}$ flask containing $(3.244 \mathrm{~g}, 5.00 \mathrm{mmol})$ of tetrakis-p-tert-butylcalix[4]arene $\mathbf{S}_{\mathbf{1}}$ and acetone $\left(50 \mathrm{~cm}^{3}\right),(1.383 \mathrm{~g}, 10.00 \mathrm{mmol})$ of $\mathrm{K}_{2} \mathrm{CO}_{3}$ were added. The mixture was stirred at room temperature for $2 \mathrm{~h}$. Then $(3.236 \mathrm{~g}, 15.00 \mathrm{mmol})$ of 1,4-butyl-dibromide in acetone $\left(50 \mathrm{~cm}^{3}\right)$ were added. The mixture was left for 4 days under reflux. After four days $5 \mathrm{~cm}^{3}$ of methanol were added. The solvents were evaporated, and the reaction mixture was dissolved in $100 \mathrm{~cm}^{3}$ of dichloromethane. After extraction with $150 \mathrm{~cm}^{3}$ of water the organic phase was dried with $\mathrm{Na}_{2} \mathrm{SO}_{4}$ and evaporated. The crude product was purified by crystallization from a 1/10 dichloromethane/methanol mixture giving (3.252 g, $3.54 \mathrm{mmol})$ of compound $\mathbf{S}_{\mathbf{2}}$ in $71 \%$ yield. $\mathrm{Mp}>280{ }^{\circ} \mathrm{C} .{ }^{1} \mathrm{H} \mathrm{NMR}\left(300 \mathrm{MHz}, \mathrm{CDCl}_{3}\right) \delta$ [ppm]: $0.98\left(\mathrm{~s}, 18 \mathrm{H}, \mathrm{C}-\left(\mathrm{CH}_{3}\right)_{3}\right), 1.30\left(\mathrm{~s}, 18 \mathrm{H}, \mathrm{C}-\left(\mathrm{CH}_{3}\right)_{3}\right), 2.15\left(\mathrm{qn}, 4 \mathrm{H}, \mathrm{J}=4.4, \mathrm{CH}_{2}-\mathrm{CH}_{2}-\right.$ O), 2.32 (qn, $4 \mathrm{H}, J=4.2, \mathrm{CH}_{2}-\mathrm{CH}_{2}-\mathrm{Br}$ ), 3.32 (d, $4 \mathrm{H}, J=13.0, \mathrm{Ar}-\mathrm{CH}_{2}-\mathrm{Ar}$ ), 3.65 (t, 4H, $J$ $\left.=6.6, \mathrm{CH}_{2}-\mathrm{CH}_{2}-\mathrm{Br}\right), 4.01\left(\mathrm{t}, 4 \mathrm{H}, J=5.8, \mathrm{CH}_{2}-\mathrm{CH}_{2}-\mathrm{O}\right), 4.25\left(\mathrm{~d}, 4 \mathrm{H}, J=13.0, \mathrm{Ar}-\mathrm{CH}_{2}-\mathrm{Ar}\right)$, 6.78 (s, 4H, Ar-H), 7.08 (s, 4H, Ar- $H$ ), 7.40 (s, 2H, OH). Anal. Calcd. for $\mathrm{C}_{52} \mathrm{H}_{70} \mathrm{O}_{4} \mathrm{Br}_{2}: \mathrm{C}$, 67.97; H, 7.68. Found: C, 68.21; H, 7.94.

\section{1,3-bis-(propoxy)-p-tert-butylcalix[4]arene $\left(\mathrm{S}_{3}\right)$}

Into a $250 \mathrm{~cm}^{3}$ flask containing $(3.244 \mathrm{~g}, 5.00 \mathrm{mmol})$ of tetrakis-p-tert-butylcalix[4]arene $\mathbf{S}_{1}$ and acetone $\left(50 \mathrm{~cm}^{3}\right),(1.383 \mathrm{~g}, 10.00 \mathrm{mmol})$ of $\mathrm{K}_{2} \mathrm{CO}_{3}$ were added. The mixture was stirred at room temperature for $2 \mathrm{~h}$. Then $(1.845 \mathrm{~g}, 15.00 \mathrm{mmol})$ of bromopropane in acetone $\left(40 \mathrm{~cm}^{3}\right)$ were added. The mixture was left for 4 days under reflux. After four days $5 \mathrm{~cm}^{3}$ of methanol were added. The solvents were evaporated, and the reaction mixture was dissolved in $100 \mathrm{~cm}^{3}$ dichloromethane. After extraction with $150 \mathrm{~cm}^{3}$ of water the organic phase was dried with $\mathrm{Na}_{2} \mathrm{SO}_{4}$ and evaporated. The crude product was purified by crystallization from a 1/9 acetone/methanol mixture giving (2.016 g, 2.75 mmol) of pure compound $\mathbf{S}_{3}$ in $55 \%$ yield. $\mathrm{Mp}>280{ }^{\circ} \mathrm{C} .{ }^{1} \mathrm{H}$ NMR $\left(200 \mathrm{MHz}, \mathrm{CDCl}_{3}\right) \delta$ [ppm]: $1.02\left(\mathrm{~s}, 18 \mathrm{H}, \mathrm{C}-\left(\mathrm{CH}_{3}\right)_{3}\right), 1.27\left(\mathrm{t}, 6 \mathrm{H}, \mathrm{J}=5.0, \mathrm{CH}_{3}-\mathrm{CH}_{2^{-}}\right), 1.28\left(\mathrm{~s}, 18 \mathrm{H}, \mathrm{C}-\left(\mathrm{CH}_{3}\right)_{3}\right)$, 
2.06 (sx, 4H, $J=4.9, \mathrm{CH}_{3}-\mathrm{CH}_{2}-\mathrm{CH}_{2}-\mathrm{O}$ ), 3.32 (d, 4H, $J=12.8$, Ar- $\mathrm{CH}_{2}-\mathrm{Ar}$ ), 3.96 (t, 4H, $J$ $\left.=5.9, \mathrm{O}-\mathrm{CH}_{2}-\mathrm{CH}_{2}\right), 4.31\left(\mathrm{~d}, 4 \mathrm{H}, J=12.8, \mathrm{Ar}-\mathrm{CH}_{2}-\mathrm{Ar}\right), 6.86(\mathrm{~s}, 4 \mathrm{H}, \mathrm{Ar}-\mathrm{H}), 7.05(\mathrm{~s}, 4 \mathrm{H}$, Ar-H), 7.89 (s, 2H, OH). Anal. Calcd for $\mathrm{C}_{50} \mathrm{H}_{68} \mathrm{O}_{4}$ : C, 81.92; H, 9.35. Found: C, 82.05; $\mathrm{H}, 9.40$.

\section{1,3-bis-(propoxy)-2,4-bis-(butoxy-4-bromide)-tetrakis-p-tert-butylcalix[4]arene $\left(\mathrm{S}_{4}\right)$}

Into a $250 \mathrm{~cm}^{3}$ flask containing $(2.016 \mathrm{~g}, 2.75 \mathrm{mmol})$ of $\mathbf{S}_{\mathbf{3}}$ and DMF $\left(50 \mathrm{~cm}^{3}\right),(0.480 \mathrm{~g}$, $20.00 \mathrm{mmol}$ ) of $\mathrm{NaH}$ were added. $\mathrm{NaH}$ was washed twice with hexane before addition. The mixture was stirred at room temperature for $4 \mathrm{~h}$. After this time, $(5.940 \mathrm{~g}, 27.5 \mathrm{mmol})$ of 1,4-dibromobutyl in DMF $\left(40 \mathrm{~cm}^{3}\right.$ ) were added. The mixture was left for 2 days at 80$90{ }^{\circ} \mathrm{C}$. After 2 days $30 \mathrm{~cm}^{3}$ of methanol were added. The solvents were evaporated and the reaction mixture was dissolved in $100 \mathrm{~cm}^{3}$ of dichloromethane. After extraction with 250 $\mathrm{cm}^{3}$ of water the organic phase was dried with $\mathrm{Na}_{2} \mathrm{SO}_{4}$ and evaporated. The crude product was purified by crystallization from a $1 / 10$ dichloromethane/methanol mixture giving $(1.584 \mathrm{~g}, 1.57 \mathrm{mmol})$ of compound $\mathbf{S}_{4}$ in $57 \%$ yield. Mp $170{ }^{\circ} \mathrm{C} .{ }^{1} \mathrm{H}$ NMR $(200 \mathrm{MHz}$, $\left.\mathrm{CDCl}_{3}\right) \delta[\mathrm{ppm}]: 1.01\left(\mathrm{t}, 6 \mathrm{H}, J=5.2, \mathrm{CH}_{3}-\mathrm{CH}_{2}\right), 1.05\left(\mathrm{~s}, 18 \mathrm{H}, \mathrm{C}-\left(\mathrm{CH}_{3}\right)_{3}\right), 1.12(\mathrm{~s}, 18 \mathrm{H}$, $\left.\mathrm{C}-\left(\mathrm{CH}_{3}\right)_{3}\right), 1.99-2.05\left(\mathrm{~m}, 4 \mathrm{H}, \mathrm{CH}_{3}-\mathrm{CH}_{2}-\right), 2.07-2.25\left(\mathrm{~m}, 8 \mathrm{H},-\mathrm{CH}_{2}-\mathrm{CH}_{2}-\mathrm{CH}_{2}-\mathrm{Br}\right), 3.13$ (d, $\left.4 \mathrm{H}, J=13.0, \mathrm{Ar}-\mathrm{CH}_{2}-\mathrm{Ar}\right), 3.51\left(\mathrm{t}, 4 \mathrm{H}, J=6.6,-\mathrm{CH}_{2}-\mathrm{Br}\right), 3.81\left(\mathrm{t}, 4 \mathrm{H}, J=5.8, \mathrm{CH}_{3}-\mathrm{CH}_{2}-\right.$ $\mathrm{CH}_{2}-\mathrm{O}$ ), 3.91 (t, $\left.4 \mathrm{H}, \mathrm{J}=5.8, \mathrm{Br}_{-} \mathrm{CH}_{2}-\mathrm{CH}_{2}-\mathrm{CH}_{2}-\mathrm{CH}_{2}-\mathrm{O}\right), 4.39$ (d, $4 \mathrm{H}, \mathrm{J}=13.0$, Ar- $\mathrm{CH}_{2}-$ Ar), $6.74(\mathrm{~s}, 4 \mathrm{H}, \mathrm{Ar}-H), 6.83(\mathrm{~s}, 4 \mathrm{H}, \mathrm{Ar}-H)$. Anal. Calcd for $\mathrm{C}_{58} \mathrm{H}_{82} \mathrm{O}_{4} \mathrm{Br}_{2}: \mathrm{C}, 69.45 ; \mathrm{H}$, 8.24. Found: C, 69.65; H, 8.30.

\section{Tetrakis-(butoxy-4-bromide)-tetrakis-p-tert-butylcalix[4]arene $\left(\mathrm{S}_{5}\right)$}

The suspension of p-tert-butylcalix[4]arene $\mathbf{S}_{\mathbf{1}}(1.947 \mathrm{~g}, 3.00 \mathrm{mmol})$ and $\mathrm{NaH}$ in oil washed three times with hexane $(0.700 \mathrm{~g}, 29.17 \mathrm{mmol})$ were stirred at room temperature in DMF $\left(50 \mathrm{~cm}^{3}\right)$ for $1 \mathrm{~h}$. Then 1,4-dibromobutane $(12.947 \mathrm{~g}, 59.06 \mathrm{mmol})$ was added and the mixture was heated to $80{ }^{\circ} \mathrm{C}$. After 4 days of heating, the mixture was cooled and $\mathrm{MeOH}\left(20 \mathrm{~cm}^{3}\right)$ was added. After removing of the solvent, the residue was dissolved in dichloromethane and water and acidified with $1 \mathrm{M} \mathrm{HCl}$. The organic layer was dried over $\mathrm{Na}_{2} \mathrm{SO}_{4}$, filtered and evaporated. After precipitation from methanol the pure compound $\mathbf{S}_{\mathbf{5}}$ ( $1.520 \mathrm{~g}, 1.28 \mathrm{mmol}$ ) was obtained in $43 \%$ yield. $\mathrm{Mp} 180{ }^{\circ} \mathrm{C} .{ }^{1} \mathrm{H} \mathrm{NMR}\left(300 \mathrm{MHz}, \mathrm{CDCl}_{3}\right)$ $\delta$ [ppm]: $1.09\left(\mathrm{~s}, 36 \mathrm{H},-\mathrm{C}\left(\mathrm{CH}_{3}\right)_{3}\right), 1.98-2.09\left(\mathrm{~m}, 8 \mathrm{H},-\mathrm{CH}_{2^{-}}\right), 2.16-2.23\left(\mathrm{~m}, 8 \mathrm{H},-\mathrm{CH}_{2^{-}}\right)$, $3.15\left(\mathrm{~d}, 4 \mathrm{H}, J=13.0, \mathrm{Ar}-\mathrm{CH}_{2}-\mathrm{Ar}\right), 3.53\left(\mathrm{t}, 8 \mathrm{H}, J=6.9,-\mathrm{CH}_{2}-\mathrm{Br}\right), 3.91(\mathrm{t}, 8 \mathrm{H}, J=6.9$, - 
$\left.\mathrm{CH}_{2}-\mathrm{O}-\right), 4.36$ (d, 4H, J = 13.0, Ar-CH$\left.-\mathrm{Ar}\right), 6.79$, (s, 8H, Ar-H). Anal. Calcd. for $\mathrm{C}_{60} \mathrm{H}_{84} \mathrm{O}_{4} \mathrm{Br}_{4}$ : C, 60.61; H, 7.12; Found: C, 60.87; H, 7.32.

\section{Tetrakis-(4-(diphenylphosphine)-butoxy)-p-tert-butylcalix[4]arene $\left(\mathrm{S}_{6}\right)$}

Into a $100 \mathrm{~cm}^{3}$ flask containing compound $\mathbf{S}_{\mathbf{5}}(1.510 \mathrm{~g}, 1.27 \mathrm{mmol})$ of freshly distilled THF $\left(10 \mathrm{~cm}^{3}\right),(1.282 \mathrm{~g}, 5.72 \mathrm{mmol})$ of $\mathrm{KP}(\mathrm{Ph})_{2}$ in THF $\left(15 \mathrm{~cm}^{3}\right)$ were added via a syringe. The mixture was stirred for 2 hours at room temperature, during which the colour of the reaction mixture changed from red to dark yellow. The mixture was then evaporated and extracted twice with $30 \mathrm{~cm}^{3}$ of dichloromethane. Purification of the crude product on silica column with a $3 / 7$ dichloromethane/hexane mixture as eluent gave compound $\mathbf{S}_{\mathbf{6}}$ (0.409 g, $0.26 \mathrm{mmol})$ in $20 \%$ yield. $\mathrm{Mp} 110{ }^{\circ} \mathrm{C} .{ }^{1} \mathrm{H}$ NMR $\left(300 \mathrm{MHz}, \mathrm{CDCl}_{3}\right) \delta[\mathrm{ppm}]$ : $1.08\left(\mathrm{~s}, 36 \mathrm{H},-\mathrm{C}\left(\mathrm{CH}_{3}\right)_{3}\right), 1.42-1.63\left(\mathrm{~m}, 8 \mathrm{H},-\mathrm{CH}_{2}-\mathrm{CH}_{2}-\mathrm{P}\right), 2.00-2.18\left(\mathrm{~m}, 8 \mathrm{H},-\mathrm{CH}_{2}-\mathrm{CH}_{2}-\right.$ O), 2.00-2.18 (m, 8H, -CH2-P), 3.05 (d, $4 \mathrm{H}, J=12.9$, Ar-CH $-\mathrm{Ar}$ ), 3.81 (t, 8H, $J=5.4$, $\mathrm{CH}_{2}-\mathrm{CH}_{2}-\mathrm{O}$ ), 4.29 (d, 4H, $J=12.9$, Ar- $\mathrm{CH}_{2}-\mathrm{Ar}$ ), 6.75 (s, 8H, Ar-H), 7.20-7.46 (m, 40H, P-Ar-H). ${ }^{31} \mathrm{P}$ NMR (400 MHz, $\left.\mathrm{CDCl}_{3}\right) \delta[\mathrm{ppm}]:-14.91$ [P]. m/z (MALDI) $1610.88(\mathrm{M}+$ $\mathrm{H})^{+}$. Anal. Calcd. for $\mathrm{C}_{108} \mathrm{H}_{124} \mathrm{O}_{4} \mathrm{P}_{4}$ : C, 80.57; H, 7.76; Found: C, 80.73; H, 7.87.

\section{Synthesis of phosphonium ligands}

\section{1,3-bis-(4-triphenylphosphonium-butoxy)-p-tert-butylcalix[4]arene dibromide (1)}

Into a $100 \mathrm{~cm}^{3}$ flask containing $\mathbf{S}_{2}(1.184 \mathrm{~g}, 2.00 \mathrm{mmol})$ in chloroform $\left(30 \mathrm{~cm}^{3}\right),(5.248 \mathrm{~g}$, $20.00 \mathrm{mmol})$ of triphenylphosphine in chloroform $\left(20 \mathrm{~cm}^{3}\right)$ were added. After 6 days under reflux, the mixture was cooled and the solvent evaporated. The residue was dissolved in dichloromethane. The excess of triphenylphosphine was precipitated from methanol and filtered out. The filtrate was evaporated. The pure product $1(2.179 \mathrm{~g}, 1.51$ mmol) was obtained by precipitation from a $1 / 9$ dichloromethane/hexane mixture, as a white-light green powder in $76 \%$ yield: $\mathrm{Mp}>280{ }^{\circ} \mathrm{C} .{ }^{1} \mathrm{H}$ NMR $\left(300 \mathrm{MHz}, \mathrm{CDCl}_{3}\right) \delta$ [ppm]: $0.99\left(\mathrm{~s}, 18 \mathrm{H},-\mathrm{C}\left(\mathrm{CH}_{3}\right)_{3}\right), 1.28\left(\mathrm{~s}, 18 \mathrm{H},-\mathrm{C}\left(\mathrm{CH}_{3}\right)_{3}\right), 2.05-2.29\left(\mathrm{~m}, 8 \mathrm{H}, \mathrm{CH}_{2}-\mathrm{CH}_{2}-\right.$ $\mathrm{CH}_{2}-\mathrm{CH}_{2}-\mathrm{O}$ ), 3.19 (d, $4 \mathrm{H}, \mathrm{J}=12.8, \mathrm{Ar}-\mathrm{CH}_{2}-\mathrm{Ar}$ ), 3.80-4.00 (m, 4H, $\left.-\mathrm{CH}_{2}-\mathrm{O}\right), 3.90$ (d, 4H, $\left.J=12.8, \mathrm{Ar}-\mathrm{CH}_{2}-\mathrm{Ar}\right), 3.90-4.08\left(\mathrm{~m}, 4 \mathrm{H},-\mathrm{CH}_{2}-\mathrm{P}\right), 6.79$ (s, 4H, Ar- $\left.\mathrm{H}\right), 6.99$ (s, 4H, Ar- $\left.\mathrm{H}\right)$, 7.49 (s, 2H, OH), 7.54 - 7.64 (m, 12H, P-Ar-H meta), 7.65-7.74 (m, 6H, P-Ar-H para), 7.80-7.93 (m, 12H, P-Ar-H ortho). ${ }^{31} \mathrm{P}$ NMR (400 MHz, $\left.\mathrm{CDCl}_{3}\right) \delta[\mathrm{ppm}]: 25.80 . \mathrm{m} / \mathrm{z}$ $\left(\mathrm{FAB}^{+}\right) 721.7(\mathrm{M}+2 \mathrm{H})^{2+} ; \mathrm{m} / \mathrm{z}(\mathrm{MALDI}) 1361.7(\mathrm{M}-\mathrm{Br})^{+}$. Anal. Calcd for $\mathrm{C}_{88} \mathrm{H}_{100} \mathrm{O}_{4} \mathrm{P}_{2} \mathrm{Br}_{2}$ : C, 73.22; H, 6.98. Found: C, 73.46; H, 7.20. 


\section{1,3-bis-(4-(P,P-diphenyl-P-methylphosphonium)-butoxy)-p-tert-butylcalix[4]arene}

\section{dibromide (2)}

Compound $\mathbf{2}$ was prepared following the same procedure as for compound $\mathbf{1}$ with $\mathbf{S}_{\mathbf{2}}$ $(2.753 \mathrm{~g}, 3.00 \mathrm{mmol})$ and diphenylmethylphosphine $(6.006 \mathrm{~g}, 30.00 \mathrm{mmol})$ in $69 \%$ yield. $\mathrm{Mp}>280{ }^{\circ} \mathrm{C} .{ }^{1} \mathrm{H}$ NMR $\left(300 \mathrm{MHz}, \mathrm{CDCl}_{3}\right) \delta[\mathrm{ppm}]: 0.96\left(\mathrm{~s}, 18 \mathrm{H},-\mathrm{C}\left(\mathrm{CH}_{3}\right)_{3}\right), 1.29(\mathrm{~s}, 18 \mathrm{H}$, $\left.-\mathrm{C}\left(\mathrm{CH}_{3}\right)_{3}\right), 1.91-2.10\left(\mathrm{~m}, 4 \mathrm{H},-\mathrm{CH}_{2}-\mathrm{CH}_{2}-\mathrm{P}\right), 2.11-2.27$ (m, 4H, $\left.-\mathrm{CH}_{2}-\mathrm{CH}_{2}-\mathrm{O}\right), 2.99$ (d, 6H, $\left.J=13.8, \mathrm{CH}_{3}-\mathrm{P}\right), 3.23\left(\mathrm{~d}, 4 \mathrm{H}, J=13.5, \mathrm{Ar}-\mathrm{CH}_{2}-\mathrm{Ar}\right), 3.58-3.75\left(\mathrm{~m}, 4 \mathrm{H},-\mathrm{CH}_{2}-\mathrm{P}\right), 3.90(\mathrm{t}$, $\left.4 \mathrm{H}, J=5.3,-\mathrm{CH}_{2}-\mathrm{O}\right), 4.02\left(\mathrm{~d}, 4 \mathrm{H}, J=13.5, \mathrm{Ar}-\mathrm{CH}_{2}-\mathrm{Ar}\right), 6.76(\mathrm{~s}, 4 \mathrm{H}, \mathrm{Ar}-\mathrm{H}), 7.01(\mathrm{~s}, 4 \mathrm{H}$, Ar-H), 7.31 (s, 2H, OH), 7.48-7.59 (m, 8 H, P-Ar-H meta), 7.61-7.70 (m, 4H, P-Ar-H para), 7.90-8.06 (m, 8H, P-Ar-H ortho). $\left.{ }^{31} \mathrm{P} \mathrm{NMR} \mathrm{(400} \mathrm{MHz,} \mathrm{CDCl}_{3}\right) \delta[\mathrm{ppm}]: 25.96 . \mathrm{m} / \mathrm{z}$ (MALDI) $1239.58(\mathrm{M}-\mathrm{Br})^{+}$. Anal. Calcd. for $\mathrm{C}_{78} \mathrm{H}_{96} \mathrm{O}_{4} \mathrm{P}_{2} \mathrm{Br}_{2}: \mathrm{C}, 71.01 ; \mathrm{H}, 7.33$. Found: C, $71.21 ; \mathrm{H}, 7.52$.

\section{1,3-bis-(4-triphenylphosphonium-butoxy)-2,4-bis-propoxy-p-tert-butyl-calix[4]arene} dibromide (3)

Into a $100 \mathrm{~cm}^{3}$ flask containing $\mathbf{S}_{\mathbf{4}}(2.012 \mathrm{~g}, 2.00 \mathrm{mmol})$ in chloroform $\left(30 \mathrm{~cm}^{3}\right), 5.248 \mathrm{~g}$ of triphenylphosphine $(20.00 \mathrm{mmol})$ in chloroform $\left(20 \mathrm{~cm}^{3}\right)$ were added and left for 6 days under reflux. After that time the mixture was cooled and the solvent evaporated. The residue was dissolved in dichloromethane. The excess of triphenylphosphine was precipitated from methanol and filtered off. The filtrate was evaporated. The pure product $3(2.179 \mathrm{~g}, 1.51 \mathrm{mmol})$ was obtained by precipitation from a $1 / 9$ dichloromethane/hexane mixture, as a white-light green powder in $76 \%$ yield. Mp $120{ }^{\circ} \mathrm{C} .{ }^{1} \mathrm{H}$ NMR $(300 \mathrm{MHz}$, $\left.\mathrm{CDCl}_{3}\right) \delta[\mathrm{ppm}]: 0.86\left(\mathrm{t}, 6 \mathrm{H}, J=5.3, \mathrm{CH}_{3}-\mathrm{CH}_{2}-\mathrm{CH}_{2}-\mathrm{O}\right), 0.99\left(\mathrm{~s}, 18 \mathrm{H},-\mathrm{C}\left(\mathrm{CH}_{3}\right)_{3}\right), 1.11$ (s, $\left.18 \mathrm{H},-\mathrm{C}\left(\mathrm{CH}_{3}\right)_{3}\right), 1.65-1.87\left(\mathrm{~m}, 4 \mathrm{H}, \mathrm{CH}_{3}-\mathrm{CH}_{2}-\mathrm{CH}_{2}-\mathrm{O}\right), 1.77-1.94\left(\mathrm{~m}, 4 \mathrm{H},-\mathrm{CH}_{2}-\mathrm{CH}_{2}-\mathrm{P}\right)$, 2.28-2.43 (m, 4H, $\left.-\mathrm{CH}_{2}-\mathrm{CH}_{2}-\mathrm{O}\right), 2.95\left(\mathrm{~d}, 4 \mathrm{H}, J=13.0\right.$, Ar- $\left.\mathrm{CH}_{2}-\mathrm{Ar}\right), 3.63(\mathrm{t}, 4 \mathrm{H}, J=5.9$, $\left.\mathrm{CH}_{3}-\mathrm{CH}_{2}-\mathrm{CH}_{2}-\mathrm{O}\right), 3.85-4.00\left(\mathrm{~m}, 4 \mathrm{H},-\mathrm{CH}_{2}-\mathrm{P}\right), 3.85-4.00\left(\mathrm{~m}, 4 \mathrm{H},-\mathrm{CH}_{2}-\mathrm{O}\right), 4.18(\mathrm{~d}, 4 \mathrm{H}, \mathrm{J}$ $\left.=13.0, \mathrm{Ar}-\mathrm{CH}_{2}-\mathrm{Ar}\right), 6.61(\mathrm{~s}, 4 \mathrm{H}, \mathrm{Ar}-\mathrm{H}), 6.76(\mathrm{~s}, 4 \mathrm{H}, \mathrm{Ar}-H), 7.60-7.92$ (m, 30H, P-Ar-H ortho, meta, para). ${ }^{31} \mathrm{P}$ NMR (400 MHz, $\left.\mathrm{CDCl}_{3}\right) \delta[\mathrm{ppm}]: 25.84 . \mathrm{m} / \mathrm{z}$ (MALDI) 1447.6 (M $-\mathrm{Br})^{+}$. Anal. Calcd. for $\mathrm{C}_{94} \mathrm{H}_{112} \mathrm{O}_{4} \mathrm{P}_{2} \mathrm{Br}_{2}$ : C, 73.91; H, 7.39. Found: C, 73.67; H, 7.66.

\section{1,3-bis-propoxy-2,4-bis-(4-(P,P-diphenyl-P-methylphosphonium)-butoxy)-p-tert- butylcalix[4]arene dibromide (4)}

Compound $\mathbf{4}$ was obtained according to the same procedure as for $\mathbf{3}$ with $\mathbf{S}_{\mathbf{4}}(3.050 \mathrm{~g}, 3.04$ 
mmol) and diphenylmethylphosphine (6.006 g, $30.00 \mathrm{mmol})$ a white powder in $64 \%$ yield. $\mathrm{Mp} 148{ }^{\circ} \mathrm{C} .{ }^{1} \mathrm{H}$ NMR $\left(300 \mathrm{MHz}, \mathrm{CDCl}_{3}\right) \delta[\mathrm{ppm}]: 0.79\left(\mathrm{~s}, 18 \mathrm{H},-\mathrm{C}\left(\mathrm{CH}_{3}\right)_{3}\right), 1.03(\mathrm{t}$, $\left.6 \mathrm{H}, \mathrm{J}=6.9, \mathrm{CH}_{3}-\mathrm{CH}_{2}-\mathrm{CH}_{2}-\mathrm{O}\right), 1.31\left(\mathrm{~s}, 18 \mathrm{H},-\mathrm{C}\left(\mathrm{CH}_{3}\right)_{3}\right), 1.55-1.75\left(\mathrm{~m}, 4 \mathrm{H},-\mathrm{CH}_{2}-\mathrm{CH}_{2}-\mathrm{P}\right)$, 1.88-2.04 (m, 4H, $\mathrm{CH}_{3}-\mathrm{CH}_{2}-\mathrm{CH}_{2}-\mathrm{O}$ ), 2.40-2.58 (m, 4H, $\mathrm{CH}_{2}-\mathrm{CH}_{2}-\mathrm{CH}_{2}-\mathrm{O}$ ), 3.05 (d, 4H, J $\left.=12.5, \mathrm{Ar}-\mathrm{CH}_{2}-\mathrm{Ar}\right), 3.23\left(\mathrm{~d}, 6 \mathrm{H}, \mathrm{J}=14.3, \mathrm{CH}_{3}-\mathrm{P}\right), 3.62-3.80\left(\mathrm{~m}, 8 \mathrm{H},-\mathrm{CH}_{2}-\mathrm{P}\right.$ and $-\mathrm{CH}_{2}-$ $\mathrm{O}$ ), 3.88 (t, $\left.4 \mathrm{H}, J=5.9,-\mathrm{CH}_{2}-\mathrm{CH}_{2}-\mathrm{CH}_{2}-\mathrm{O}\right), 4.32$ (d, $4 \mathrm{H}, J=12.5, \mathrm{Ar}-\mathrm{CH}_{2}-\mathrm{Ar}$ ), 6.43 (s, 4H, Ar-H), 7.08 (s, 4H, Ar-H), 7.60-7.80 (m, 12H, P-Ar-H meta, para), 8.01-8.13 (m, 8H, P-Ar-H ortho). ${ }^{31} \mathrm{P}$ NMR (400 MHz, $\left.\mathrm{CDCl}_{3}\right) \delta[\mathrm{ppm}]: 25.98 . \mathrm{m} / \mathrm{z}$ (MALDI) 1323.7 (M $\mathrm{Br})^{+}$. Anal. Calcd. for $\mathrm{C}_{84} \mathrm{H}_{108} \mathrm{O}_{4} \mathrm{P}_{2} \mathrm{Br}_{2}$ : C, 71.88; H, 7.76. Found: C, 71.99; H, 7.85.

\section{Tetrakis-(4-triphenylphosphonium-butoxy)-tetrakis-p-tert-butylcalix[4]arene} tetrabromide (5)

Compound $\mathbf{S}_{\mathbf{5}}(1.184 \mathrm{~g}, 1.00 \mathrm{mmol})$ was dissolved in chloroform $\left(30 \mathrm{~cm}^{3}\right)$. After a few minutes of stirring triphenylphosphine $(5.248 \mathrm{~g}, 20.00 \mathrm{mmol})$ and chloroform $\left(20 \mathrm{~cm}^{3}\right)$ were added. After 6 days of refluxing the mixture was cooled and solvent was evaporated. The residue was dissolved in dichloromethane. The excess of triphenylphosphine was precipitated from methanol and filtered. The organic layer was evaporated. Chromatography on a silica column with 90:10 dichloromethane: methanol mixture as eluent gave compound 5 (0.67 g, $0.30 \mathrm{mmol})$ in $30 \%$ yield. Mp $132{ }^{\circ} \mathrm{C} .{ }^{1} \mathrm{H}$ NMR (300 $\left.\mathrm{MHz} ; \mathrm{CDCl}_{3}\right) \delta[\mathrm{ppm}]: 1.02\left(\mathrm{~s}, 36 \mathrm{H},-\mathrm{C}\left(\mathrm{CH}_{3}\right)_{3}\right), 1.56-1.72\left(\mathrm{~m}, 8 \mathrm{H},-\mathrm{CH}_{2^{-}}\right), 2.24-2.41(\mathrm{~m}$, $\left.8 \mathrm{H},-\mathrm{CH}_{2}-\right), 2.91\left(\mathrm{~d}, 4 \mathrm{H}, \mathrm{J}=13.0, \mathrm{Ar}-\mathrm{CH}_{2}-\mathrm{Ar}\right), 3.78-4.01$ (m, 16H, $-\mathrm{CH}_{2}-\mathrm{P}$ and Ar-O$\mathrm{CH}_{2}$ ), 4.23 (d, 4H, $\left.J=13.0, \mathrm{Ar}-\mathrm{CH}_{2}-\mathrm{Ar}\right), 6.63$ (s, 8H, Ar-H), 7.59-7.71- (m, 36H, P-Ar- H, meta, para), 7.76-7.88 (m, 24H, P-Ar- $H$, ortho). ${ }^{31} \mathrm{P}$ NMR (400 MHz, $\left.\mathrm{CDCl}_{3}\right) \delta[\mathrm{ppm}]$ : 25.76. $\mathrm{m} / \mathrm{z}\left(\mathrm{FAB}^{+}\right) 479.5(\mathrm{M})^{4+} ; \mathrm{m} / \mathrm{z}(\mathrm{MALDI}) 2157.7(\mathrm{M}-\mathrm{Br})^{+}$. Anal. Calcd. for $\mathrm{C}_{132} \mathrm{H}_{144} \mathrm{O}_{4} \mathrm{P}_{4} \mathrm{Br}_{4}$ : C, 70.84; H, 6.49. Found: C, 70.97; H, 6.69.

\section{Tetrakis-(4-triphenylphosphonium-butoxy)-p-tert-butylcalix[4]arene tetraperchlorate} (5a)

In a $10 \mathrm{~cm}^{3}$ flask $5(0.100 \mathrm{~g}, 0.045 \mathrm{mmol})$ was dissolved in acetonitrile $\left(1 \mathrm{~cm}^{3}\right) .(0.050 \mathrm{~g}$, $0.241 \mathrm{mmol})$ of $\mathrm{AgClO}_{4}$ in acetonitrile $\left(1 \mathrm{~cm}^{3}\right)$ were added dropwise to the ligand solution. After 24 hours of stirring at room temperature, the precipitate of $\mathrm{AgBr}$ was filtered off. The filtrate was evaporated to give compound $\mathbf{5 a}(0.088 \mathrm{~g}, 0.038 \mathrm{mmol})$ in 84 $\%$ yield. $\mathrm{Mp}>120{ }^{\circ} \mathrm{C}$ decomposition. ${ }^{1} \mathrm{H}$ NMR $\left(300 \mathrm{MHz}, \mathrm{CDCl}_{3}\right) \delta[\mathrm{ppm}]: 1.07(\mathrm{~s}, 36 \mathrm{H}$, $\left.-\mathrm{C}\left(\mathrm{CH}_{3}\right)_{3}\right), 1.70-1.85\left(\mathrm{~m}, 8 \mathrm{H},-\mathrm{CH}_{2}-\mathrm{CH}_{2}-\mathrm{P}\right), 2.45-2.53\left(\mathrm{~m}, 8 \mathrm{H},-\mathrm{CH}_{2}-\mathrm{CH}_{2}-\mathrm{O}\right), 2.95$ (d, 4H, 
$J=13.0$, Ar- $\left.\mathrm{CH}_{2}-\mathrm{Ar}\right), 3.43-3.60\left(\mathrm{~m}, 8 \mathrm{H},-\mathrm{CH}_{2}-\mathrm{P}\right), 4.20-4.30\left(\mathrm{~m}, 8 \mathrm{H},-\mathrm{CH}_{2}-\mathrm{O}\right), 4.44(\mathrm{~d}$, $\left.4 \mathrm{H}, J=13.0, \mathrm{Ar}-\mathrm{CH}_{2}-\mathrm{Ar}\right), 6.97(\mathrm{~s}, 8 \mathrm{H}, \mathrm{Ar}-\mathrm{H}), 7.56-7.90(\mathrm{~m}, 60 \mathrm{H}, \mathrm{P}-\mathrm{Ar}-\mathrm{H}$, ortho, meta, para), ${ }^{31} \mathrm{P}$ NMR (400 MHz, $\left.\mathrm{CDCl}_{3}\right) \delta[\mathrm{ppm}]: 24.54 \mathrm{~m} / \mathrm{z}$ (MALDI) $2214.2\left(\mathrm{M}-\mathrm{ClO}_{4}\right)^{+}$. Anal. Calcd: for $\mathrm{C}_{132} \mathrm{H}_{144} \mathrm{O}_{4} \mathrm{P}_{4}\left(\mathrm{ClO}_{4}\right)_{4}$ : C, 68.45; H, 6.27. Found: C, 68.70; H, 6.45.

Tetrakis-(4-triphenylphosphonium-butoxy)-tetrakis-p-tert-butylcalix[4]arene tetrahexafluorophosphate $(5 b)$

Compound 5 (0.100 g, 0.045 mmoles) was dissolved in acetonitrile $\left(1 \mathrm{~cm}^{3}\right)$. Then $(0.062$ $\mathrm{g}, 0.245 \mathrm{mmol}) \mathrm{AgPF}_{6}$ were dissolved in acetonitrile and added dropwise to the ligand solution. After $24 \mathrm{~h}$ the precipitate of $\mathrm{NaBr}$ was removed and the solution was evaporated. Compound 5 b (0.090 g, 0.036 mmole) was obtained in $80 \%$ yield. Mp $128{ }^{\circ} \mathrm{C} .{ }^{1} \mathrm{H}$ NMR $\left(500 \mathrm{MHz} ; \mathrm{CDCl}_{3}\right) \delta[\mathrm{ppm}]: 1.12\left(\mathrm{~s}, 36 \mathrm{H},-\mathrm{C}\left(\mathrm{CH}_{3}\right)_{3}\right), 1.65-1.81\left(\mathrm{~m}, 8 \mathrm{H},-\mathrm{CH}_{2^{-}}\right), 2.38-2.49$ (m, 8H, - $\mathrm{CH}_{2^{-}}$), 3.25-3.38 (m, 8H, $\left.-\mathrm{CH}_{2}-\mathrm{P}\right), 3.47$ (d, 4H, $J=13.0$, Ar- $\left.\mathrm{CH}_{2}-\mathrm{Ar}\right), 4.15-4.24$ (m, 8H, Ar-O-CH $\mathrm{CH}_{2}, 4.47$ (d, 4H, $J=13.0, \mathrm{Ar}-\mathrm{CH}_{2}-\mathrm{Ar}$ ), 7.04 (s, 8H, Ar- $H$ ), 7.66-7.70 (m, $60 \mathrm{H}, \mathrm{P}-\mathrm{Ar}-H) .{ }^{31} \mathrm{P} \mathrm{NMR}\left(400 \mathrm{MHz}, \mathrm{CDCl}_{3}\right) \delta[\mathrm{ppm}]: 24.72\left[\mathrm{P}^{+}\right],-143.39\left[\mathrm{PF}_{6}\right] . \mathrm{m} / \mathrm{z}$ (MALDI) $1447.60\left(\mathrm{M}-\mathrm{PF}_{6}\right)^{+}$. Anal. Calcd. for $\mathrm{C}_{132} \mathrm{H}_{144} \mathrm{O}_{4} \mathrm{P}_{4}\left(\mathrm{PF}_{6}\right)_{4}: \mathrm{C}, 63.46 ; \mathrm{H}, 5.81$. Found: C, 63.30; H, 5.78.

\section{Tetrakis-(4-(P,P-diphenyl-P-methylphosphonium)-butoxy)-p-tert-butylcalix[4]arene} tetrabromide (6)

Into a $100 \mathrm{~cm}^{3}$ flask containing $\mathbf{S 5}(1.184 \mathrm{~g}, 1.00 \mathrm{mmol})$ dissolved in chloroform (30 $\left.\mathrm{cm}^{3}\right)$, (4.004 g, $\left.20.00 \mathrm{mmol}\right)$ diphenylmethylphosphine in chloroform $\left(20 \mathrm{~cm}^{3}\right)$ were added. After 6 days under reflux the mixture was cooled and the solvent was evaporated. The product was purified by precipitation from a 1/9 dichloromethane/hexane mixture to give compound $6(1.190 \mathrm{~g}, 0.59 \mathrm{mmol})$ in $59 \%$ yield. Mp $160{ }^{\circ} \mathrm{C} .{ }^{1} \mathrm{H}$ NMR $(300 \mathrm{MHz}$; $\left.\mathrm{CDCl}_{3}\right) \delta[\mathrm{ppm}]: 1.03\left(\mathrm{~s}, 36 \mathrm{H},-\mathrm{C}\left(\mathrm{CH}_{3}\right)_{3}\right), 1.55-1.75\left(\mathrm{~m}, 8 \mathrm{H},-\mathrm{CH}_{2}-\mathrm{CH}_{2}-\mathrm{P}\right), 2.30-2.48(\mathrm{~m}$, $\left.8 \mathrm{H},-\mathrm{CH}_{2}-\mathrm{CH}_{2}-\mathrm{O}\right), 2.91$ (d, $\left.12 \mathrm{H}, J=13.5, \mathrm{CH}_{3}-\mathrm{P}\right), 3.03$ (d, 4H, J = 12.8, Ar-CH $-\mathrm{Ar}$ ), 3.70-3.87 (m, 16H, - $\mathrm{CH}_{2}-\mathrm{P}$ and $\left.\mathrm{CH}_{2}-\mathrm{O}\right), 4.26$ (d, 4H, $J=12.8, \mathrm{Ar}-\mathrm{CH}_{2}-\mathrm{Ar}$ ), 6.71 (s, 8H, Ar-H), 7.54-7.78 (m, 24H, P-Ar-H meta, para), 8.02-8.17 (m, 16H, P-Ar-H ortho). ${ }^{31} \mathrm{P}$ NMR (400 MHz, $\left.\mathrm{CDCl}_{3}\right) \delta[\mathrm{ppm}]: 25.98 . \mathrm{m} / \mathrm{z}$ (MALDI) $1909.68(\mathrm{M}-\mathrm{Br})^{+}$. Anal. Calcd. for $\mathrm{C}_{112} \mathrm{H}_{136} \mathrm{O}_{4} \mathrm{P}_{4} \mathrm{Br}_{4}$ : C, 67.61; H, 6.89. Found: C, 68.59; H, 7.10.

Tetrakis-(4-(P,P-diphenyl-P-methylphosphonium)-butoxy)-p-tert-butylcalix[4]arene 


\section{tetraperchlorate $(6 a)$}

Compound 6a was obtained according to the same procedure as 5a with $6(0.100 \mathrm{~g}, 0.05$ mmol) and $\mathrm{AgClO}_{4}(0.050 \mathrm{~g}, 0.24 \mathrm{mmol})$ in $90 \%$ yield. $\mathrm{Mp}>120{ }^{\circ} \mathrm{C}$ decomposition. ${ }^{1} \mathrm{H}$ NMR (300 MHz, $\left.\mathrm{CDCl}_{3}\right) \delta$ [ppm]: 1.09 (s, 36H, $\left.-\mathrm{C}\left(\mathrm{CH}_{3}\right)_{3}\right), 1.65-1.80\left(\mathrm{~m}, 8 \mathrm{H},-\mathrm{CH}_{2}-\mathrm{CH}_{2}-\right.$ P), 2.35-2.50 (m, 8H, $\left.-\mathrm{CH}_{2}-\mathrm{CH}_{2}-\mathrm{O}\right), 2.50\left(\mathrm{~d}, 12 \mathrm{H}, \mathrm{J}=12.0, \mathrm{CH}_{3}-\mathrm{P}\right), 3.18-3.28(\mathrm{~m}, 8 \mathrm{H},-$ $\left.\mathrm{CH}_{2}-\mathrm{P}\right), 3.38\left(\mathrm{~d}, 4 \mathrm{H}, \mathrm{J}=12.8, \mathrm{Ar}-\mathrm{CH}_{2}-\mathrm{Ar}\right), 4.15-4.28\left(\mathrm{~m}, 8 \mathrm{H},-\mathrm{CH}_{2}-\mathrm{O}\right), 4.45(\mathrm{~d}, 4 \mathrm{H}, J=$ 12.8, Ar- $\mathrm{CH}_{2}$-Ar), 6.97 (s, 8H, Ar-H), 7.60-7.87 (m, 40H, P-Ar-H, ortho, meta, para). ${ }^{31} \mathrm{P}$ NMR (400 MHz, $\left.\mathrm{CDCl}_{3}\right) \delta[\mathrm{ppm}]:$ 24.77. m/z (MALDI) $1966.24\left(\mathrm{M}-\mathrm{ClO}_{4}\right)^{+}$. Anal. Calcd. for $\mathrm{C}_{112} \mathrm{H}_{136} \mathrm{O}_{4} \mathrm{P}_{4}\left(\mathrm{ClO}_{4}\right)_{4}$ : C, 65.05; H, 6.63. Found: C, 65.13; H, 6.72.

\section{Tetrakis-(4-(P,P-diphenyl-P-methylphosphonium)-butoxy)-p-tert-butylcalix[4]arene} tetrahexafluorophosphate $(6 \mathrm{~b})$

Compound $\mathbf{6 b}$ was obtained according to the same procedure as for $5 \mathbf{b}$ with $\mathbf{6}(0.100 \mathrm{~g}$, $0.05 \mathrm{mmol})$ and $\mathrm{AgPF}_{6}(0.069 \mathrm{~g}, 0.27 \mathrm{mmol})$ in $80 \%$ yield. Mp $155{ }^{\circ} \mathrm{C} .{ }^{1} \mathrm{H}$ NMR (300 $\left.\mathrm{MHz}, \mathrm{CDCl}_{3}\right) \delta[\mathrm{ppm}]: 1.11\left(\mathrm{~s}, 36 \mathrm{H},-\mathrm{C}\left(\mathrm{CH}_{3}\right)_{3}\right), 1.55-1.70\left(\mathrm{~m}, 8 \mathrm{H},-\mathrm{CH}_{2}-\mathrm{CH}_{2}-\mathrm{P}\right), 2.24-$ $2.38\left(\mathrm{~m}, 8 \mathrm{H},-\mathrm{CH}_{2}-\mathrm{CH}_{2}-\mathrm{O}\right), 2.42\left(\mathrm{~d}, 12 \mathrm{H}, \mathrm{J}=12.0, \mathrm{CH}_{3}-\mathrm{P}\right), 2.95-3.10\left(\mathrm{~m}, 8 \mathrm{H},-\mathrm{CH}_{2}-\mathrm{P}\right)$, 3.39 (d, $4 \mathrm{H}, J=12.8$, Ar- $\mathrm{CH}_{2}$-Ar), 4.05-4.20 (m, 8H, $\left.-\mathrm{CH}_{2}-\mathrm{O}\right), 4.42$ (d, 4H, $J=12.8$, Ar$\mathrm{CH}_{2}$-Ar), 7.00 (s, 8H, Ar-H), 7.58-7.80 (m, 40H, P-Ar-H, ortho, meta, para). ${ }^{31} \mathrm{P}$ NMR $\left(400 \mathrm{MHz}, \mathrm{CDCl}_{3}\right) \delta[\mathrm{ppm}]: 24.50\left[\mathrm{P}^{+}\right],-143.07\left[\mathrm{PF}_{6}\right], \mathrm{m} / \mathrm{z}(\mathrm{MALDI}) 2104.80\left(\mathrm{M}-\mathrm{PF}_{6}\right)^{+}$. Anal. Calcd. for $\mathrm{C}_{112} \mathrm{H}_{136} \mathrm{O}_{4} \mathrm{P}_{4}\left(\mathrm{PF}_{6}\right)_{4}$ : C, 59.79; H, 6.09. Found: C, 59.87; H, 6.03.

\section{Protonated tetrakis-(4-(P,P-diphenyl-phosphine)-butoxy)-p-tert-butylcalix[4]arene} tetrabromide (7)

Into a $50 \mathrm{~cm}^{3}$ flask containing $\mathbf{S}_{\mathbf{6}}(1.510 \mathrm{~g}, 0.94 \mathrm{mmol})$ in dichloromethane $\left(10 \mathrm{~cm}^{3}\right), 5$ $\mathrm{cm}^{3}$ of $\mathrm{HBr}$ (solution of $33 \%$ wt. in glacial acetic acid) in dichloromethane $\left(15 \mathrm{~cm}^{3}\right)$ were added. The mixture was left stirred for 24 hours at room temperature. The mixture was then evaporated to give the pure product $7(0.954 \mathrm{~g}, 0.49 \mathrm{mmol})$ in $52 \%$ yield. Mp 175 ${ }^{\circ} \mathrm{C} .{ }^{1} \mathrm{H}$ NMR $\left(300 \mathrm{MHz}, \mathrm{CDCl}_{3}\right) \delta$ [ppm]: 1.22 (s, 36H, -C(CH3) $), 1.80-2.15(\mathrm{~m}, 16 \mathrm{H}, \mathrm{O}-$ $\mathrm{CH}_{2}-\mathrm{CH}_{2}-\mathrm{CH}_{2}-\mathrm{CH}_{2}-\mathrm{P}$ ), 2.92-3.10 (m, $4 \mathrm{H}, \mathrm{Ar}-\mathrm{CH}_{2}-\mathrm{Ar}$ ), 3.18-3.30 (m, 8H, $\mathrm{CH}_{2}-\mathrm{O}$ ), 3.40$3.55\left(\mathrm{~m}, 8 \mathrm{H},-\mathrm{CH}_{2}-\mathrm{P}\right), 4.05-4.27$ (m, 4H, Ar- $\mathrm{CH}_{2}-\mathrm{Ar}$ ), 7.05 (s, 8H, Ar- $\mathrm{H}$ ), 7.60-8.05 (m, 40H, P-Ar-H, ortho, meta, para), 10.30-10.40 (m, 4H, H-P). ${ }^{31} \mathrm{P}$ NMR (400 MHz, $\mathrm{CDCl}_{3}$ ) $\delta$ [ppm]: $46.32\left[\mathrm{P}^{+}\right] . \mathrm{m} / \mathrm{z}$ (MALDI) $1849.04(\mathrm{M}-\mathrm{Br})^{+}$. Anal. Calcd. for $\mathrm{C}_{108} \mathrm{H}_{128} \mathrm{O}_{4} \mathrm{P}_{4} \mathrm{Br}_{4}$ : C, 67.08; H, 6.67. Found: C, 66.84; H, 6.92. 


\section{NMR studies}

$5 \mathrm{mg}$ of ligand were introduced with 6 equivalents of the solid alkali metal salts $\left(\mathrm{Li}^{+}, \mathrm{Na}^{+}\right.$, $\mathrm{Cs}^{+}$) or tetraethylammonium perchlorate in a glass vessel and dissolved in a small volume of deuterated chloroform or acetonitrile. After manual shaking for a few minutes, the mixture was left in contact for 24 hours before filtration of the excess of salt if necessary. The solution (or the filtrate) was then sampled in a NMR tube and its spectrum recorded on Bruker SY300 $\mathrm{MHz}$ or SY400 MHz spectrometers equipped for ${ }^{1} \mathrm{H}$ or ${ }^{31} \mathrm{P}$ resonances.

\section{Microcalorimetric studies}

Microcalorimetric titrations were performed using a 2277 Thermal Activity Monitor Microcalorimeter (Thermometric). Titration were carried out at $25{ }^{\circ} \mathrm{C}$ on $2.7 \mathrm{~cm}^{3}$ of $10^{-5}$ to $5 \times 10^{-4} \mathrm{M}$ solutions of the ligand in acetonitrile using a glass cell of $4 \mathrm{~cm}^{3}$. The heat changes were measured after injection of $15 \times 15 \mu \mathrm{L}$ of $10^{-3}$ and $10^{-2} \mathrm{M} \mathrm{LiClO}_{4}, \mathrm{LiBr}, \mathrm{NaClO}_{4}, \mathrm{NaPF}_{6}$, or $\mathrm{Et}_{4} \mathrm{NClO}_{4}$ solutions in the same solvent. Chemical calibration was made by determination of the complexation enthalpy of $\mathrm{Ba}^{2+}$ with $18 \mathrm{C} 6$ in water or of $\mathrm{Rb}^{+}$with $18 \mathrm{C} 6$ in methanol, as recommended [37]. Values of the stability constants $(\beta)$ and of the enthalpies of complexation $(\Delta H)$ were refined simultaneously from these data using the ligand binding analysis program DIGITAM version 4.1 [38] and after correction for the heat of dilution determined in separate experiments by adding the salt solutions to $2.7 \mathrm{~cm}^{3}$ of pure solvent. The values of the corresponding entropies of complexation $(\Delta S)$ were then derived from the expressions $\Delta G=-R T \ln \beta$ and $\Delta G=\Delta H-T \Delta S$.

\section{Ion selective electrodes}

THF was dried and freshly distilled before used for the preparation of the ion selective membranes. PVC (high molecular weight poly(vinyl chloride), 2-nitrophenyl octyl ether (o-NPOE), bis-(2-ethylhexyl)sebacate (BEHS), (2-morpholino)ethanesulfonic acid monohydrate (MES) were from Fluka Selectophore. The $\mathrm{LiClO}_{4}, \mathrm{CsClO}_{4}$ and sodium salts: $\mathrm{Cl}^{-}, \mathrm{Br}^{-}, \mathrm{I}^{-}, \mathrm{ClO}_{4}{ }^{-}, \mathrm{SCN}^{-}, \mathrm{NO}_{3}{ }^{-}, \mathrm{SO}_{4}{ }^{2-}, \mathrm{CO}_{3}{ }^{2-}, \mathrm{HPO}_{4}{ }^{2-}, \mathrm{PO}_{4}{ }^{3-}, \mathrm{Cr}_{2} \mathrm{O}_{7}{ }^{2-}$, citrate, acetate, benzoate, and oxalate were of p.a. grade. All aqueous salt solutions were prepared with demineralised water (conductivity $<1.0 \mu \mathrm{S} / \mathrm{cm}$ ).

The membranes were composed of $4 \mathrm{mg}$ of ionophores $\mathbf{1}-\mathbf{6}$ and $\mathbf{8}, 60 \mathrm{mg}$ of PVC and 120 $\mathrm{mg}$ of plasticizer. All the components were dissolved in $1.5 \mathrm{~cm}^{3}$ of dried, freshly distilled 


\section{Acknowledgements}

R.P. gratefully acknowledges the Gdansk University of Technology, Poland, and the Ministère de la Recherche et des Nouvelles Technologies, France (Cotutelles de thèses), for financial support. 


\section{References}

[1] Bianchi, A.; Bowman-James, K.; Garcia-Espana, E. Eds., Supramolecular Chemistry of Anions; John Wiley-VCH, New York, 1997, ch. 3, pp. 63.

[2] Kanyo, Z. F.; Christianson, D. W. J. Biol. Chem., 1991, 266, 4264.

[3] Pardee, A. P. J. Biol. Chem., 1966, 241, 5887.

[4] Puljak L.; Kilic, G. Biochim. Biophys. Acta, 2006, 1762, 404.

[5] Weiner, M. L.; Salminen, W. F.; Larson, P. R.; Barter, R. A.; Kranetz J. L.; Simon, G. S. Food and Chemical Toxicology, 2001, 39, 759.

[6] Matzer, W. E. Environmental Forensic, 2001, 2, 301.

[7] Bondy, C. R.; Gale P. A.; Loeb, S. J. J. Am. Chem. Soc., 2004, 126, 5030.

[8] Amendola, V.; Boiocchi, M.; Esteban-Gómez, D.; Fabbrizzi L.; Monzani, E. Org. Biomol. Chem., 2005, 3, 2632.

[9] Brooks, S. J.; Edwards, P. R.; Gale P. A.; Light, M. E. New J. Chem., 2006, 30, 65.

[10] Turner, D. R.; Paterson M. J.; Steed, J. W. J. Org. Chem., 2006, 71, 1598.

[11] Boerrigter, H.; Grave, L.; M. Nissink, J. W.; Chrisstoffels, L. A. J.; van der Maas, J. H.; Verboom, W.; de Jong F.; Reinhoudt, D. N.; J. Org. Chem., 1998, 63, 4174.

[12] Lee K. H.; Hong, J. I. Tetrahedron Lett., 2000, 41, 6083.

[13] ChmielewskiM.; Jurczak, J. Tetrahedron Lett., 2004, 45, 6007.

[14] Beer, P. D.; Drew M. G. B.; Gradwell, . K. J. Chem. Soc., Perkin Trans. 2, 2000, 511.

[15] Dietrich, B. Pure \& Appl. Chem., 1993, 65, 1457.

[16] Egorov, V. V.; Rakhman'ko, E. M.; Okaev E. B.; Pomelenok E. V.; Nazarov, V. A.; Talanta, 2004, 63, 119.

[17] Matthews S. E.; Beer, P. D. Calixarenes 2001; Asfari, Z.; Böhmer, V.; Harrowfield, J.; Vicens J. Eds., Kluwer Academic Publishers, Dordrecht, 2001, ch. 23, p. 421.

[18] Miao, R.; Zheng, Q. Y.; Chen C. F.; Huang Z. T. Tetrahedron Lett., 2004, 45, 4959. 
[19] Jagessar, R. C.; Shang, M.; Scheidt, W. R.; Burns; D. H. J. Am. Chem. Soc., 1998, $120,11684$.

[20] Gale, P. A.; Sessler, J. L.; Kral, V.; Lynch, V. J. Am. Chem. Soc., 1996, 118, 5140.

[21] Gale, P. A.; Sessler, J. L.; Allen, W. E.; Tvermoes, N. A.; Lynch, V. Chem. Commun., 1997, 665.

[22] Pomecko, R.; Asfari, Z.; Hubscher-Bruder, V.; Bochenska M.; Arnaud-Neu, F. Supramol. Chem., 2007, 19, 459.

[23] Hamdi, A.; Nam, K. C.; Ryu, B.J.; Kim, J. S.; Vicens, J. Tetrahedron Letters 2004, 45, 4689 .

[24] Broder, C. K.; Davidson, M. G.; Forsyth, V. T.; Howard, J. A. K.; Lamb S.; Mason, S. A. Crys. Grow. Des., 2002, 3, 163.

[25] Issleib K.; Muller, D.-W. Chem. Berichte, 1959, 92, 3175.

[26] Clark, P. W. Org. Prep. and Proc. Int., 1979, 11, 103.

[27] Mohr, W.; Horn, C. R.; Stahl J.; Gladysz, J. A. Synthesis, 2003, 8, 1279.

[28] Baklouti, L.; Cherif J.; Abidi, R.; Arnaud-Neu, F.; Harrowfield J.; Vicens, J. Org. Biomol. Chem., 2004, 2, 2786.

[29] Savage, P. B.; Holmgren S. K.; Gellman, S. H. J. Am. Chem. Soc., 1994, 116, 4069.

[30] Casabo, J.; Munoz J. A.; Errachid, A. Sens. Actuators, B, 1997, 43, 206.

[31] Shamsipur, M.; Soleymanpour, A.; Akhond, M.; Sharghi H.; Hasaninejad, A. R. Sens. Actuators, B, 2003, 89, 9 .

[32] Dinten, O.; Spichiger, U. E.; Chaniotakis, N.; Gehrig, P.; Rusterholz, B.; Morf, W. E.; Simon, W. Anal. Chem., 1991, 63, 596.

[33] Lizondo-Sabater, J.; Segui, M. J.; Loris, J. M.; Martinez-Manez, R.; Pardo, T.; Sancenon, F.; Soto, J. Sens. Actuators B, 2004, 101, 20.

[34] Segui, M. J.; Lizondo-Sabater, J.; Martinez-Manez, R.; Soto, J. Analyst, 2002, 127, 387.

[35] Casabo, J.; Escriche, L.; Perez-Jimenez, C.; Munoz, J. A.; Teixidor, F.; Baussells, J.; Errachid, A. Anal. Chim. Acta, 1996, 320, 63.

[36] Schaller, U.; Bakker, E.; Spichiger, U. E.; Pretsch, E. Anal. Chem., 1994, 66, 391.

[37] Arnaud-Neu, F.; Delgado, R.; Chaves, S. Pure Appl. Chem., 2003, 75, 71.

[38] Hallen, D. Pure Appl. Chem., 1993, 65, 1527.

[39] Bochenska, M.; Zielenska, A.; Pomecko, R.; Hubscher-Bruder, V.; Arnaud-Neu, F. J. Incl. Phenom., 2005, 52, 129. 
[40] Buck, R. P.; Linder, E. Pure Appl. Chem., 1994, 66, 2527.

[41] Umezawa, Y.; Buhlmann, P.; Umezawa, K.; Tohda, K.; Amemiya, S. Pure Appl. Chem. 2000, 72, 1851.

[42] Lindner, E.; Umezawa, Y. Pure Appl. Chem. 2008, 80, 85. 
Table 1. Changes ( $\Delta \delta$ in ppm) in the ${ }^{31} \mathrm{P}$ NMR spectra of phosphonium ligands in the presence of sodium iodide, thiocyanate and perchlorate in $\mathrm{CDCl}_{3}$

\begin{tabular}{ccccc}
\hline & $\mathbf{1}$ & $\mathbf{2}$ & $\mathbf{5}$ & $\mathbf{6}$ \\
\hline Ligand $(\delta)$ & 25.797 & 25.962 & 25.756 & 25.982 \\
Ligand $+\mathrm{I}^{-}(\Delta \delta)$ & 0.215 & 0.203 & 0.210 & 0.175 \\
Ligand $+\mathrm{SCN}^{-}(\Delta \delta)$ & 0.470 & 0.530 & 0.767 & 0.965 \\
Ligand $+\mathrm{ClO}_{4}^{-}(\Delta \delta)$ & 0.602 & 0.620 & 0.992 & 1.170 \\
\hline
\end{tabular}


Table 2. Differences $(\Delta \delta)$ in the proton chemical shifts $(\delta)[\mathrm{ppm}]$ in the spectra of ligands 5 and $6,5 \mathbf{a}$ and $6 \mathbf{a}, 5 \mathbf{b}$ and $6 \mathbf{b}$ in $\mathrm{CD}_{3} \mathrm{CN}$

\begin{tabular}{|c|c|c|c|c|c|c|}
\hline & c & $\mathbf{e}$ & $\mathbf{f}$ & $\mathbf{g}$ & $\mathbf{j}$ & o \\
\hline$\delta(5)$ & 6.87 & 2.93 & 4.15 & 3.74 & 3.81 & - \\
\hline$\delta(5 a)$ & 6.83 & 2.91 & 4.11 & 3.76 & 3.17 & - \\
\hline$\Delta \delta(5 \mathbf{a}-5)$ & -0.04 & -0.02 & -0.04 & 0.02 & -0.64 & - \\
\hline$\delta(5 b)$ & 6.80 & 2.87 & 4.09 & 3.77 & 3.06 & - \\
\hline$\Delta \delta(5 \mathrm{~b}-5)$ & -0.07 & -0.06 & -0.06 & 0.03 & -0.75 & - \\
\hline$\delta(6)$ & 6.92 & 3.02 & 4.19 & 3.70 & 3.58 & 2.78 \\
\hline$\delta(\mathbf{6 a})$ & 6.89 & 2.98 & 4.15 & 3.73 & 2.85 & 2.37 \\
\hline$\Delta \delta(6 a-6)$ & -0.03 & -0.04 & -0.04 & 0.03 & -0.73 & -0.41 \\
\hline$\delta(6 \mathbf{b})$ & 6.88 & 2.97 & 4.12 & 3.75 & 2.75 & 2.32 \\
\hline$\Delta \delta(6 \mathrm{~b}-6)$ & -0.04 & -0.05 & -0.07 & 0.05 & -0.83 & -0.46 \\
\hline
\end{tabular}


Table 3. Characteristics of potentiometric responds for perchlorate of PVC/NPOE electrodes containing ligand $\mathbf{5}$ and $\mathbf{6}$ and different amount of KTCIPB. (Inner and conditioning electrolyte $\mathrm{MES} / \mathrm{NaOH} \mathrm{pH}=5.5 / 10^{-2} \mathrm{M} \mathrm{NaCl}$ )

\begin{tabular}{cccc}
\hline Ligand & $\begin{array}{c}\text { KTClPB } \\
(\mathrm{mol} \%)\end{array}$ & $\begin{array}{c}\mathrm{S} \\
(\mathrm{mV} / \mathrm{dec} \text { de })\end{array}$ & $\begin{array}{c}\text { LR } \\
(\log [\mathrm{A}])\end{array}$ \\
\hline $\mathbf{5}$ & 0 & -55.6 & -6.0 \\
$\mathbf{5}$ & 40 & -54.6 & -6.0 \\
$\mathbf{5}$ & 120 & -55.9 & -5.7 \\
$\mathbf{6}$ & 0 & -54.2 & -6.0 \\
$\mathbf{6}$ & 40 & -51.8 & -6.0 \\
$\mathbf{6}$ & 120 & -49.9 & -5.7 \\
\hline
\end{tabular}


Table 4. Characteristics of potentiometric responds for perchlorate of PVC/NPOE electrodes containing tetrasubstituted phosphonium ligands with different counterions (Inner and conditioning electrolyte $\mathrm{MES} / \mathrm{NaOH} \mathrm{pH}=5.5 / 10^{-2} \mathrm{M}$ $\mathrm{NaCl})$.

\begin{tabular}{cccc}
\hline Ligand & Counterion & $\begin{array}{c}\mathrm{S} \\
(\mathrm{mV} / \mathrm{dec} \text { de })\end{array}$ & $\begin{array}{c}\mathrm{LR} \\
(\log [\mathrm{A}])\end{array}$ \\
\hline $\mathbf{5}$ & $\mathrm{Br}^{-}$ & -56.5 & -6.0 \\
$\mathbf{5 a}$ & $\mathrm{ClO}_{4}^{-}$ & -56.1 & -6.0 \\
$\mathbf{5 b}$ & $\mathrm{PF}_{6}^{-}$ & -38.3 & -5.5 \\
$\mathbf{6}$ & $\mathrm{Br}^{-}$ & -54.4 & -5.7 \\
$\mathbf{6 a}$ & $\mathrm{ClO}_{4}^{-}$ & -40.4 & -6.0 \\
$\mathbf{6 b}$ & $\mathrm{PF}_{6}^{-}$ & -36.2 & -6.0 \\
\hline
\end{tabular}


Table 5. Selectivity coefficients as $\log \boldsymbol{K}_{\mathrm{ClO}_{4}^{-}, \mathrm{X}}^{\text {pot }}$ of the PVC/NPOE membrane electrodes based on phosphonium calixarenes $\mathbf{1}$ - $\mathbf{6}$ and the monomer $\mathbf{8}$

\begin{tabular}{cccccccc}
\hline & \multicolumn{7}{c}{$\log \boldsymbol{K}_{\mathbf{C l O}_{4}^{-}, X}^{\text {pot }}$} \\
Anion X & $\mathbf{1}$ & $\mathbf{2}$ & $\mathbf{3}$ & $\mathbf{4}$ & $\mathbf{5}$ & $\mathbf{6}$ & $\mathbf{8}$ \\
\cline { 2 - 7 } & 0 & 0 & 0 & 0 & 0 & 0 & 0 \\
$\mathrm{ClO}_{4}{ }^{-}$ & -1.2 & -1.4 & -1.1 & -0.6 & -1.3 & -1.4 & -1.1 \\
$\mathrm{SCN}^{-}$ & -1.7 & -1.7 & -1.3 & -0.9 & -2.0 & -2.0 & -1.6 \\
$\mathrm{I}^{-}$ & -2.6 & -2.6 & -2.4 & -1.9 & -2.9 & -3.0 & -2.5 \\
$\mathrm{NO}_{3}{ }^{-}$ & -4.6 & -4.5 & -4.6 & -4.4 & -4.6 & -4.4 & nd \\
$\mathrm{HCO}_{3}{ }^{-}$ & -2.2 & -2.4 & -2.6 & -1.7 & -2.6 & -3.2 & -1.9 \\
$\mathrm{Cr}_{2} \mathrm{O}_{7}{ }^{2-}$ & -4.5 & -4.5 & -4.5 & -4.4 & -4.5 & -4.4 & nd \\
$\mathrm{HPO}_{4}{ }^{2-}$ & -4.9 & -4.7 & -4.7 & -4.7 & -4.7 & -4.5 & nd \\
$\mathrm{SO}_{4}{ }^{2-}$ & & & & & & &
\end{tabular}

nd: not determined 


\section{Figure captions}

Figure 1. Chemical structures of the ligands under study

Figure 2. $\quad{ }^{1} \mathrm{H}$ NMR spectra of ligand $\mathbf{1}$ alone and in the presence of $\mathrm{ClO}_{4}{ }^{-}$in $\mathrm{CDCl}_{3}$

Figure 3. $\quad{ }^{1} \mathrm{H}$ NMR spectra of ligand 6 alone and in the presence of $\mathrm{ClO}_{4}{ }^{-}$in $\mathrm{CDCl}_{3}$

Figure 4. Potentiometric anion responses of electrodes with PVC/NPOE membrane containing ligand 1 in MES buffer at $\mathrm{pH}$ 5.5.

Figure 5. Potentiometric anion responses of electrodes with PVC/NPOE membrane containing ligand 6 in MES buffer at $\mathrm{pH}$ 5.5. 


\section{Anion recognition by phosphonium calix[4]arenes : synthesis and physico- chemical studies}

Radoslaw Pomecko, ${ }^{\mathrm{a}, \mathrm{b}}$ Zouhair Asfari, ${ }^{\mathrm{a}}$ Véronique Hubscher-Bruder, ${ }^{\mathrm{a}}$ Maria Bochenska ${ }^{\mathrm{b} \text { }}$ and Françoise Arnaud-Neu ${ }^{a^{*}}$

${ }^{a} I P H C-D S A, U L P, C N R S, E C P M, 25$ rue Becquerel, 67087 Strasbourg Cedex 2, France; Email:farnaud@chimie.u-strasbg.fr

${ }^{b}$ Department of Chemical Technology, Chemical Faculty, Gdansk University of Technology, ul. Narutowicza 11/12,80-264 Gdansk,Poland.E-mail: marboch@chem.pg.gda.pl

p-tert-Butylcalix[4]arenes, in the cone conformation, di- and tetrasubstituted at the narrow rim with charged phosphonium groups, have been synthesized and characterized. Their interactions with a wide range of anions have been investigated in solution in chloroform and acetonitrile by means of ${ }^{1} \mathrm{H}$ and ${ }^{31} \mathrm{P}$ NMR and microcalorimetry (ITC). These compounds have also been incorporated as sensing material in PVC ion selective electrodes (ISE). The results showed that they interact strongly with the more lipophilic anions $\mathrm{ClO}_{4}^{-}, \mathrm{SCN}^{-}$and $\mathrm{I}^{-}$, in solution as in the electrode membranes. The origin of this selectivity is discussed and, in particular, the role of the salt counterion is examined.

Keywords: Phosphonium calix[4]arenes; anion binding properties; microcalorimetry; ion selective electrodes.

\section{INTRODUCTION}

Anions play an important role in many biological processes, such as regulation of cell activity, synthesis of proteins, transport of hormones $[1,2,3]$. They are also frequently used in many industrial technologies, which very often generate an increase of the concentration of anions in the environment or even introduce anionic species which were unknown so far in ecosystems. The presence of these anions is crucial in environmental and medical concerns, as they are pollutants and may have harmful effects on living organisms and human health $[4,5,6]$. Therefore there is a need of fast and selective anion detection methods allowing real-time monitoring of anion concentration changes and of efficient clean up processes. Design of anion receptors for such applications remains a great challenge for chemists because they have to take into consideration the specific 
anion properties, such as a large range of shapes and geometries, small electric charges $v s$. sizes, high free energies of solvation, and in some cases multiple oxidation states of the central atoms in oxoanions or $\mathrm{pH}$ dependence. In many artificial anion hosts, noncovalent interactions are responsible for host-guest recognition. They include electrostatic interactions, hydrogen bonding, hydrophobic effects, coordination to a metal ion or combinations of these interactions. The hosts can be neutral, containing urea $[7,8,9,10]$, thiourea $[11,12]$ or amide functions [13]. They can also be positively charged, containing pyridinium [14], polyammonium [15] or quaternary ammonium [16] binding sites. Calix[4]arenes [17,18] and porphyrins [19] are often used as scaffolds onto which these functional groups can be grafted. Calixpyrroles are also known as efficient anion receptors $[20,21]$.

Recently, we described the synthesis and characterization of a new calix[4]arene derivative (5) bearing four positively charged triphenyl phosphonium groups [22]. The presence of these highly polarisable moieties, where the charge is spread over the three aromatic rings, was expected to favour the interaction with lipophilic anions. Preliminary binding studies showed that this ligand interacted selectively with some anions, namely $\mathrm{ClO}_{4}{ }^{-}$and $\mathrm{SCN}^{-}$. This compound was also incorporated, as ionophore-sensing material, in ion selective electrodes (ISEs) which exhibited a selectivity order similar to the Hofmeister series. The disubstituted derivative $\mathbf{1}$ was also synthesised as the hexafluorophosphate [23].

In order to get more information on the mechanisms involved in the recognition process and to optimize its selectivity, we have now extended this study to new di- and tetrasubstituted phosphonium calix[4]arene derivatives (compounds $\mathbf{2 - 4 , 6}$ and 7) and reexamined the properties of 1 and 5 (Fig. 1). In some of these compounds, one of the phenyl rings on the phosphonium groups has been replaced by a methyl radical or a hydrogen atom. The presence of such small substituents is expected to increase the charge density on the phosphorus atoms and their accessibility [24]. Moreover, the presence of hydrogen atoms may induce the formation of hydrogen bonds with anions. The possibility of tuning the charge density on the phosphorus atoms by changing the nature of the their substituents could allow the design of receptors able to distinguish lipophilic anions like $\mathrm{ClO}_{4}^{-}, \mathrm{SCN}^{-}, \mathrm{I}^{-}$or $\mathrm{NO}_{3}^{-}$from other anions and between them. The binding properties of these compounds towards a variety of anions have been followed by ${ }^{1} \mathrm{H}$ and ${ }^{31} \mathrm{P}$ NMR and by titration microcalorimetry (ITC). In particular the role of the salt counterion was examined using these techniques. 


\section{RESULTS AND DISCUSSION}

\section{Synthesis}

The ligands synthesised in this work (Fig. 1) are based on a tetrakis-p-tertbutylcalix[4]arene platform which presents several advantages. It has a well defined size and is readily amenable to substitution at its lower rim where ligating groups can be attached and in some extent preorganized. The amphiphilicity of such derivatives should allow their introduction into the membranes of ion selective electrodes.

Diphosphonium ligands were synthesised in two or three steps according to the known procedure [23]. The first step was the selective bromoalkylation of the tetrakis-p-tertbutylcalix[4] arene $\left(\mathbf{S}_{1}\right)$, leading to the intermediate molecules $\mathbf{S}_{2}$ or $\mathbf{S}_{3}$ in $71 \%$ and $55 \%$ yield, respectively (Scheme 1). 1,3-bis-(4-triphenylphosphonium-butoxy)-p-tert-butylcalix[4]arene dibromide (1) was obtained by the reaction of $\mathbf{S}_{2}$ with 10 equivalents of triphenylphosphine. After 6 days under reflux in chloroform, the product was precipitated from a dichloromethane/hexane mixture in 76\% yield. 1,3-bis-(4-(P,P-diphenyl-Pmethylphosphonium)-butoxy)-p-tert-butylcalix[4]arene dibromide (2) was obtained during the reaction of $\mathbf{S}_{\mathbf{2}}$ with 10 equivalents of diphenylmethylphosphine in the same conditions in $69 \%$ yield.

\section{Scheme 1}

The more lipophilic molecules $\mathbf{3}$ and $\mathbf{4}$, where the free phenolic protons are substituted with propyl groups, were prepared in order to increase their stability in the lipophilic membrane of ion selective electrodes. The reaction of $\mathbf{S}_{3}$ with 10 equivalents of 1,4dibromobutyl, refluxed for 4 days in dimethylformamide in the presence of 7 equivalents of $\mathrm{NaH}$ gave the 1,3-bis-(propoxy)-2,4-bis-(butoxy-4-bromide)-tetrakis-p-tert-butylcalix[4]arene $\left(\mathbf{S}_{\mathbf{4}}\right)$ in $57 \%$ yield. Compounds 3 and $\mathbf{4}$ were obtained by the reaction of $\mathbf{S}_{4}$ with 10 equivalents of triphenylphosphine and 10 equivalents of diphenylmethylphosphine in chloroform, in 76\% and 64\% yield, respectively (Scheme 1).

Tetraphosphonium ligands $\mathbf{6}$ and $\mathbf{7}$ were synthesised according to the procedure already described for 5 [22] from the intermediate molecule tetrakis-(butoxy-4-bromide)-p-tertbutylcalix[4]arene $\left(\mathbf{S}_{5}\right)$ by substitution of the bromine atoms of the alkyl chains (Scheme 2). The tetrakis-(4-(P,P-diphenyl-P-methylphosphonium)-butoxy)-p-tert-butylcalix[4]arene tetrabromide (6) was obtained from the reaction of $\mathbf{S}_{5}$ with 20 equivalents of diphenylmethylphosphine in chloroform with 59\% yield. 


\section{Scheme 2}

Ligand 7 was synthesised in three steps. The reaction of $\mathbf{S}_{5}$ with 5 equivalents of $\mathrm{KPPh}_{2}$ gave the product $\mathbf{S}_{\mathbf{6}}$ in $40 \%$ yield [25,26,27]. $\mathbf{S}_{\mathbf{6}}$ was then protonated with an excess of $\mathrm{HBr}$ giving ligand 7 in $52 \%$ yield.

In order to study the influence of the ligand counterion on the ligand - anion interactions, the tetra substituted phosphonium ligands were also synthesised as perchlorates (5a, 6a) and hexafluorophosphates $(\mathbf{5 b}, \mathbf{6 b})$. These compounds were obtained by reacting ligands $\mathbf{5}$ and $\mathbf{6}$ with the appropriate silver salts.

For comparison purpose, the monomeric subunit of ligand 5, the triphenylphosphoniumbutoxy-p-tert-butylphenol bromide (8) was synthesized as previously described [22].

The cone conformation of the di-substituted calix[4]arenes was indicated by the presence in their ${ }^{1} \mathrm{H}$ NMR spectra of two singlets for the tert-butyl protons (at 0.99 and 1.28 ppm for 1 and 0.96 and 1.29 ppm for 2) and a AB system for the methylene protons (at 3.19 and $3.90 \mathrm{ppm}$ for $\mathbf{1}$ and at 3.23 and $4.02 \mathrm{ppm}$ for 2 ). The ${ }^{1} \mathrm{H}$ NMR spectra of ligands 5 and $\mathbf{6}$ are characteristic of tetrasubstituted derivatives of calix[4]arenes in the cone conformation. For instance in the case of $\mathbf{6}$, it is indicated by the presence of the singlet corresponding to the protons of the tert-butyl groups at $1.03 \mathrm{ppm}$, the $\mathrm{AB}$ system of the methylene protons of the calixarene at 4.26 and $3.03 \mathrm{ppm}$. Two well defined multiplets for the protons of the aromatic phosphonium groups can be observed as well as one doublet for the methyl protons in direct neighbourhood of the phosphorus atoms.

The spectrum of the protonated phosphonium ligand 7 presents several broad peaks corresponding to multiplets in coalescence. Only two singlets could be clearly observed, one for the tert-butyl groups and one for the aromatic protons of the calix[4]arene, suggesting also the cone conformation of this molecule.

\section{Binding studies \\ ${ }^{1} \mathbf{H}$ and ${ }^{31} \mathbf{P}$ NMR studies in chloroform}

The interactions between the phosphonium calixarenes and the following anions: $\mathrm{NO}_{3}{ }^{-}$, $\mathrm{ClO}_{4}^{-}, \mathrm{I}^{-}, \mathrm{SCN}^{-}, \mathrm{SO}_{4}{ }^{2-}, \mathrm{HCO}_{3}^{-}, \mathrm{Cr}_{2} \mathrm{O}_{7}{ }^{2-}$ provided as sodium salts were studied by ${ }^{1} \mathrm{H}$ and ${ }^{31} \mathrm{P}$ NMR in $\mathrm{CDCl}_{3}$. Among these anions, only perchlorate, thiocyanate and iodide salts induced changes in the ${ }^{1} \mathrm{H}$ NMR spectra of the ligands. The changes observed in the case of the disubstituted ligand $\mathbf{1}$ upon addition of sodium perchlorate are illustrated in Fig. 2. The main signals affected were those of the $\mathrm{CH}_{2}$ protons $(\mathrm{i}, \mathrm{j})$ directly bound to the carbon 
atoms next to the phosphorus atoms and the aromatic protons $(\mathrm{k})$ of the phosphonium groups (see Table S1, Supplementary Online Material). The shifts of these signals due to changes in the charge density on the phosphorus atoms reflect interactions with these anions.

\section{Figure 2}

Similar but smaller changes were induced in the spectrum of ligand 2 by the presence of the same anions (see Table S2, Supplementary Online Material). As with ligand 1, no change is observed for the aromatic protons (c, d) and for the protons (e, f) of the methylene bridge, indicating that the conformation of the calixarene unit is not disturbed. This may be explained by the high rigidity of these disubstituted derivatives due to hydrogen bonds involving the free phenolic groups.

In the ${ }^{31} \mathrm{P}$ NMR spectra of the free ligands $\mathbf{1}$ and $\mathbf{2}$, and of these ligands in the presence of sodium iodide, thiocyanate and perchlorate, the phosphorus atoms appear as a singlet indicating that the two phosphonium groups are chemically equivalent and participate in the anion-ligand interaction. The most important changes in chemical shifts are observed for perchlorate: $\Delta \delta=0.602$ and $0.620 \mathrm{ppm}$ with $\mathbf{1}$ and $\mathbf{2}$, respectively (Table 1).

\section{Table 1}

With the tetrasubstituted calix[4]arene 5 previously studied [22] and $\mathrm{ClO}_{4}^{-}, \mathrm{SCN}^{-}$and $\mathrm{I}^{-}$ the most important changes of chemical shifts corresponded to the signals of the methylene bridge protons and the aromatic protons of the calixarene, indicating changes in the conformational tensions of the calixarene scaffold. The signals corresponding to protons of the butyl chains were also shifted as well as the aromatic protons of the phosphonium. On the contrary no change was observed with the monomer 8 [22].

The spectrum of ligand $\mathbf{6}$ was also modified in the presence of these anions (see Table S3, Supplementary Online Material). In particular the signals corresponding to the protons of the calix[4]arene scaffold are moved in a comparable way for the three anions. The changes observed in the spectrum of this ligand in the presence of $\mathrm{ClO}_{4}{ }^{-}$are illustrated in Fig. 3.

\section{Figure 3}

With this anion, the multiplet corresponding to the protons (g) and (j) from 3.87 to $3.70 \mathrm{ppm}$ gives two multiplets from 4.36 to $4.17 \mathrm{ppm}$ for (g) and from 3.28 to $3.11 \mathrm{ppm}$ for (j). The multiplet from 8.17 to $8.02 \mathrm{ppm}$ for protons $(\mathrm{k})$ is shifted upfield and gives one multiplet from 7.90 to $7.70 \mathrm{ppm}$, whereas the multiplet from 7.78 to $7.54 \mathrm{ppm}$ of protons $(1, \mathrm{~m})$ does not move significantly. The doublet of the $\mathrm{CH}_{3}$ protons (o) adjacent to the phosphorus atoms at $2.91 \mathrm{ppm}$ is strongly shifted to $2.50 \mathrm{ppm}$. 
The addition of the anions studied to 7 did not induce any change in its ${ }^{1} \mathrm{H}$ NMR spectrum. Especially the signals of the protons of the phosphonium moieties, expected to be involved in hydrogen bond formation, were not shifted.

With ligand $\mathbf{6}$ as with 5, only one singlet for the phosphorus atoms was detected in its ${ }^{31} \mathrm{P}$ NMR spectrum indicating all the four phosphonium groups being chemically equivalent. In the presence of sodium perchlorate and thiocyanate, the changes in chemical shifts are larger than those observed with the disubstituted derivatives and suggest stronger interactions (Table 1). With all ligands the most important values were observed for perchlorate.

\section{${ }^{1} \mathbf{H}$ NMR studies in acetonitrile}

${ }^{1} \mathrm{H}$ NMR experiments were repeated with 5 and sodium perchlorate in deuterated acetonitrile, a more dissociating solvent, in which association phenomena are not as important as in chloroform [22]. The spectra of the ligand are very similar in both solvents. In acetonitrile the addition of $\mathrm{NaClO}_{4}$ induces shifts of the same signals as in chloroform. Moreover this study showed the influence of the counterion of the salts on the shifts observed in the spectra (See Table S4, Supplementary Online Material). The signal of the methylene protons ( $\mathrm{g}$ ) adjacent to the phenolic oxygen atoms was shifted only with $\mathrm{NaClO}_{4}$ and $\mathrm{LiClO}_{4}$. With both salts the shifts corresponding to the signals of the protons (j) next to the phosphorus atoms (which are supposed to interact with perchlorate) were similar, whereas with $\mathrm{Et}_{4} \mathrm{NClO}_{4}$ and especially with $\mathrm{CsClO}_{4}$, the values were very small. The changes observed suggested that ligand - anion interaction was connected with the nature of the counterion and its affinity for the ligand.

In order to observe the influence of the ligand counterion, bromide anions were replaced by the more lipophilic perchlorate (5a and $\mathbf{6 a}$ ) or hexafluorophosphate (5b and 6b) anions. The chemical shifts $(\delta)$ of selected protons, given in Table 2 , show only slight differences for protons (c, e, f) and (g) close to the calix[4]arene scaffold ( $\Delta \delta$ in the range $0.02-0.07 \mathrm{ppm})$. In contrast, the signals of the protons $(\mathrm{j})$ next to the charged phosphorus atoms are greatly shifted $(\Delta \delta=-0.64 \mathrm{ppm}$ for $\mathbf{5 a},-0.75 \mathrm{ppm}$ for $5 \mathbf{b}$ and $\Delta \delta=-0.73 \mathrm{ppm}$ for $\mathbf{6 a},-0.83 \mathrm{ppm}$ for $\mathbf{6 b}$ ). With ligands $\mathbf{6 a}$ and $\mathbf{6 b}$, the signals of the protons (o) of the methyl substituents are also displaced $(\Delta \delta=-0.41 \mathrm{ppm}$ for $\mathbf{6 a}$ and $-0.46 \mathrm{ppm}$ for $\mathbf{6 b})$. These results show that the chemical shifts of the protons next to the phosphonium groups are influenced by the ligand counterion.

Table 2 
On the other hand, it was also shown that when the lipophilic $\mathrm{PF}_{6}{ }^{-}$anions were replacing the $\mathrm{Br}^{-}$counterions of the ligand, there was still a significant shift of the protons (j) close to the phosphorus atoms for ligand $\mathbf{5 b}$ in the presence of $\mathrm{ClO}_{4}{ }^{-}$(Table S4) [22]. This result suggested complexation of perchlorate with this ligand, where no exchange is normally possible.

\section{Microcalorimetric studies in acetonitrile}

In order to get more information on the influence of the counterion of the salt, microcalorimetric titrations were carried out with ligands 5 and $\mathbf{5 b}$ against $\mathrm{NaClO}_{4}$, $\mathrm{LiClO}_{4}$ and $\mathrm{Et}_{4} \mathrm{NClO}_{4}$ in acetonitrile. The thermograms recorded during the titration of these ligands with $\mathrm{NaClO}_{4}$ and $\mathrm{LiClO}_{4}$ showed significant exothermic heat effects, whereas their titration with $\mathrm{Et}_{4} \mathrm{NClO}_{4}$ led to no thermal effect (see Figure $\mathrm{S} 1$, Supplementary Online Material). For comparison purpose, the titration of the monomer 8 with $\mathrm{NaClO}_{4}$ was also carried out showing no significant heat effect.

If only the $\mathrm{ClO}_{4}{ }^{-}$anion were involved in the complexation, a similar heat effect should be observed in all the titrations. The fact that an effect is only observed for $\mathrm{NaClO}_{4}$ and $\mathrm{LiClO}_{4}$ suggests that it is not only related to the anion interaction (complexation or anion exchange). Assuming that the large tetraethylammonium cation cannot be complexed with a calix[4] arene, the heat effects observed during the titration with $\mathrm{NaClO}_{4}$ and $\mathrm{LiClO}_{4}$ would rather be due to the complexation of the cations with the two ligands. This is supported by the fact that no heat effect was detected with the monomer 8, supposed to be unable to complex these cations.

Calorimetric data obtained with $\mathrm{NaClO}_{4}$ and $\mathrm{LiClO}_{4}$ were interpreted assuming different cation complexation models. With sodium and both ligands, the best fit was obtained by considering the presence of $\mathrm{ML}$ and $\mathrm{ML}_{2}$ complexes. The same species were found with $\mathrm{LiClO}_{4}$ and $\mathbf{5 b}$, whereas only a 1:1 complex was formed with $\mathbf{5}$. The formation of $\mathrm{ML}_{2}$ species could be explained by the complexation of the ion pair $\mathrm{Na}^{+} \mathrm{ClO}_{4}{ }^{-}$by two ligands. The stability constants of these complexes are given in Table S5 (see Supplementary Online Material).

An important heat effect was observed during the titration of ligands $\mathbf{5}$ and $\mathbf{5 b}$ against $\mathrm{LiBr}$, which should be related directly to cation complexation as no anion exchange is possible with these ligands. The data interpretation led to species of the same stoichiometry as with $\mathrm{LiClO}_{4}$ (Table S5). The values of the stability constants of 1:1 species formed in the presence of $\mathrm{LiClO}_{4}$ and $\mathrm{LiBr}$ are comparable (with $5, \log \beta=3.24$ 
and 3.15, respectively, and with $5 \mathbf{b}, \log \beta=4.29$ and 4.32 , respectively). They are lower than that of the complex formed by the tetra-methylated $p$-tert-butylcalix[4]arene with lithium $(\log \beta=5.10$ in acetonitrile [28]).

All that considered, the UV spectrophotometric titrations of ligand 5 against $\mathrm{ClO}_{4}{ }^{-}$ previously performed may certainly be interpreted in terms of cation rather than anion complexation [22]. The values of the stability constants of the 1:1 complexes with sodium perchlorate $(\log \beta=3.81 \pm 0.02)$ and with lithium perchlorate $(\log \beta=3.71 \pm 0.04)$ [22] are of the same order of magnitude as those obtained from microcalorimetric measurements.

\section{Potentiometric studies}

Only few ligands containing phosphorus atoms have been studied so far as active material in ion selective membrane electrodes [29,30,31]. They showed a selective response for $\mathrm{ClO}_{4}^{-}$, with, however, little discrimination with respect to $\mathrm{I}^{-}$and $\mathrm{SCN}^{-}$. The results suggested a particular affinity of ligands containing phosphorus atoms for $\mathrm{ClO}_{4}{ }^{-}, \mathrm{SCN}^{-}$and $\mathrm{I}^{-}$anions. By attaching phosphonium moieties to a calix[4]arene scaffold and taking advantage of the preorganization of the ligand, it was expected to enhance the selectivity for tetrahedral or spherical anions. Such selectivity (especially for $\mathrm{ClO}_{4}^{-}$over $\mathrm{I}^{-}$) is hard to obtain with other kinds of receptors.

Disubstituted phosphonium ligands $\mathbf{1}$ and $\mathbf{2}$ were tested as ionophores in the membrane electrodes. The electrodes were sensitive to perchlorate, thiocyanate, iodide and nitrate, showing fast, near-Nernstian responses (Figure 4 and Table S6 of Supplementary Online Material). However, their characteristics changed with time. Attempts to optimize the composition of the conditioning solutions as well as the conditioning time did not improve the situation which might be due to slow leakage of the ionophores from the membrane. These ligands had also the tendency to crystallize in the membrane phase. Crystallization of our ligands within the membranes depends strongly on the kind of plasticizer used. The ligands in the membranes based on bis-(2ethylhexyl)sebacate (BEHS) has crystallized strongly, which decreased their stability. This phenomenon originates from the higher lipophilicity of this plasticizer $(\log P=10.1)$ as compared to 2-nitrophenyloctylether (o-NPOE) $(\log P=5.9)$ [32], which is more suitable for charged ligands. Electrodes with membranes based on o-NPOE had the best lifetime and response characteristics and were chosen for further studies. 


\section{Figure 4}

The more lipophilic ligands 3 and $\mathbf{4}$, in which n-propyl chains replace the two phenolic $\mathrm{OH}$ groups, were also synthesised. The lifetime of electrodes incorporating these ligands for perchlorate was increased to at least three weeks. The repeatability of the measurements was also good, but their detection limits increased (see Table S7, Supplementary Online Material).

The membranes of electrodes incorporating the tetrasubstituted ligand $\mathbf{6}$ showed rather quick (within 15-20 s), stable and fully reversible responses (Figure 5 and Table S8 of Supplementary Online Material). The repeatability of the measurements was also good and their lifetime was more than three weeks. They showed close to Nernstian response to $\mathrm{ClO}_{4}{ }^{-}, \mathrm{I}^{-}, \mathrm{NO}_{3}{ }^{-}$and $\mathrm{SCN}^{-}$and no significant response for $\mathrm{SO}_{4}{ }^{2-}, \mathrm{CO}_{3}{ }^{2-}, \mathrm{HPO}_{4}{ }^{2-}, \mathrm{PO}_{4}{ }^{3-}$. A similar behaviour was already observed with electrodes incorporating ligand 5 (Table S8). The highest selectivity was obtained for $\mathrm{ClO}_{4}{ }^{-}$ions in buffered solution $(\mathrm{pH}=5.5)$ and in water $(\mathrm{pH}=6.5)$. The over-Nernstian slope of the electrode response for $\mathrm{Cr}_{2} \mathrm{O}_{7}{ }^{2-}$ could indicate a mechanism where both processes, anion complexation and anion exchange, play an important role. It can also be explained as the presence in the sample of different forms of chromates.

\section{Figure 5}

While the addition of lipophilic anionic sites (KTClPB) to the membranes in the case of ligand 5 did not change much the properties of the electrodes, it affected the properties of the electrodes containing 6 (Table 3). Without salt, the slope of the electrode is $-54.2 \mathrm{mV}$, and slightly decreases to -51.8 and $-49.9 \mathrm{mV}$, respectively, in the presence of salt. According to the literature data [29,31], such results suggest that none of the ligands works as a neutral carrier because the addition of the lipophilic anion to the membrane does not induce a cationic response of the potentiometric cell. Ligand 6 seems to work in the membrane as a typical anion-exchanger, whereas ligand $\mathbf{5}$ could be considered as a charged ligand despite the small influence of KTClPB.

\section{Table 3}

The influence of the ligand counterions $\left(\mathrm{Br}^{-}, \mathrm{ClO}_{4}{ }^{-}, \mathrm{PF}_{6}{ }^{-}\right)$on the properties of the membrane electrodes was also studied. Table 4 compares the responses to perchlorate of electrodes based on ligands 5a, 6a (perchlorates) and on ligands $5 \mathbf{b}$ and $\mathbf{6 b}$ (hexafluorophosphates) to corresponding electrodes based on ligands 5 and $\mathbf{6}$ (bromides). The less good slope of the electrodes containing $\mathbf{6 a}$ and $\mathbf{6 b}$ as compared to that of electrodes containing 6 suggests rather the anion-exchange nature of the latter ligand. In 
this case the presence of more lipophilic anions (perchlorate or hexafluorophosphate) slows down the anion exchange process. In contrast, the properties of electrodes with the bromide ligand 5 and with the perchlorate ligand 5a are comparable. The presence of highly lipophilic perchlorate anions does not disturb the electrode response, while the presence of hexafluorophosphate anions in the membrane phase (electrode containing ligand $5 \mathbf{b})$ decreases the slope and the linearity range.

\section{Table 4}

The poorer properties of the electrode containing $\mathbf{5 b}$ and $\mathbf{6 b}$ could be explained by the higher lipophilicity of hexafluorophosphate anions which hinders the process of anion exchange. The complexation of $\mathrm{ClO}_{4}{ }^{-}$by ligand 5 could explain the good response of electrodes based on 5 and 5a to perchlorate. Such interpretation is consistent with the results of previous experiments (Table 4) and indicates that ligand 5 behaves more like a charged carrier for $\mathrm{ClO}_{4}{ }^{-}$, while ligand 6 behaves more as an anion-exchanger.

The order of selectivity observed with all phosphonium ligands 1-6 and 8 follows the Hofmeister series:

$$
\mathrm{ClO}_{4}{ }^{-}>\mathrm{SCN}^{-}>\mathrm{I}^{-}>\mathrm{Cr}_{2} \mathrm{O}_{7}{ }^{2-}>\mathrm{NO}_{3}{ }^{-}>\mathrm{Br}^{-}>\mathrm{HCO}_{3}{ }^{-}>\mathrm{HPO}_{4}{ }^{2-}>\mathrm{SO}_{4}{ }^{2-}
$$

The highest selectivity is observed for perchlorate (Table 5).

\section{Table 5}

The electrodes based on tetraphosphonium derivatives show higher selectivities for perchlorate over thiocyanate, iodide and nitrate than those based on their di-phosphonium counterparts. These selectivities are also better than the selectivity of electrodes based on the monomer 8 .

The replacement of one phenyl substituent on the phosphorus atoms by one methyl group does not change significantly the selectivity pattern and the values of the selectivity coefficients of electrodes containing either di- or tetraphosphonium ligands. The only exception is the selectivity of electrodes based on compound 5 against $\mathrm{Cr}_{2} \mathrm{O}_{7}{ }^{2-}$ which increases from 2.6 to $3.2 \log$ units.

Electrodes based on alkylated compounds $\mathbf{3}$ and $\mathbf{4}$ are also selective for perchlorate but the selectivity over iodide and thiocyanate is decreased (Table 5).

Ligands 5 and 6 display better potentiometric properties than the protonated cyclam [33] or its copper complex [34] and than a phosphodithia macrocycle [35]. For instance the detection limit is $2.5 \times 10^{-7} \mathrm{M}$ with ligand 5 when compared to $4.2 \times 10^{-6} \mathrm{M}$ for cyclam and $8 \times 10^{-7} \mathrm{M}$ for the phosphodithiamacrocycle. Ligands 6 as 5 presents generally higher selectivities than TDMACl [36], the protonated cyclam and $[\mathrm{Cu}(\mathrm{cyclam})]^{2+}$. 


\section{CONCLUDING REMARKS}

The different techniques used to assess the binding properties of phosphonium derivatives showed strong interactions with $\mathrm{SCN}^{-}, \mathrm{I}^{-}$and especially $\mathrm{ClO}_{4}^{-}$and pointed out the important role played by the salt counterion $\left(\mathrm{Na}^{+} \mathrm{or} \mathrm{Li}^{+}\right)$, which may be complexed by the calix[4]arene. Incorporated in PVC membrane electrodes, these molecules are efficient sensing material for anions with a selectivity order following the Hofmeister series generally observed for ion exchangers. However, the electrodes based on tetraphosphonium derivatives showed better selectivities than those based on the diphosphonium analogues or on the monomeric unit, indicating the importance of the ligand preorganisation which should not be observed in the case of simple anionexchangers.

A question which must be addressed concerns the nature of the interaction between the ligands and the anions, e.g. $\mathrm{ClO}_{4}^{-}$. Is it a simple ion-exchange between the bromides of the ligand and this more lipophilic anion, or is it complexation within the charged phosphonium groups? What is the exact role of the salt counterion?

If NMR gives some indications on the changes in the molecule, suggesting interactions, it does not tell if there is complexation or anion exchange, since the nature of the counterion of the ligand has been shown to influence the chemical shifts of the protons near the charged atoms. In favour of ion-exchange is the fact that the most important shifts are observed with the more lipophilic anions $\mathrm{ClO}_{4}^{-}, \mathrm{SCN}^{-}$and $\mathrm{I}^{-}$. The behaviour in selective electrodes is consistent also with this assumption. However, the fact that no change occurred in the spectrum of the monomer, where only exchange is possible, is against this hypothesis. In favour of complexation is the fact that, in the presence of $\mathrm{NaClO}_{4}$, strong shifts are observed for the signal of the protons next to the phosphonium groups in the spectrum of the hexafluorophosphate ligand where no exchange is possible. On the other hand ${ }^{1} \mathrm{H}$ NMR and microcalorimetry emphasized the importance of the cation which can be complexed in the cavity of the calixarene. With perchlorate, the best interaction takes place with $\mathrm{Na}^{+}$and $\mathrm{Li}^{+}$, whereas little or no interaction occurs with the larger $\mathrm{Et}_{4} \mathrm{~N}^{+}$and $\mathrm{Cs}^{+}$. It can be noted also that the anion plays also a role in the complexation of the cation, since the spectrum of the calixarene part is not affected in the presence of $\mathrm{NO}_{3}{ }^{-}, \mathrm{SO}_{4}{ }^{2-} \mathrm{HCO}_{3}{ }^{-}$and $\mathrm{Cr}_{2} \mathrm{O}_{7}{ }^{2-}$, i.e. the less lipophilic ones.

\section{EXPERIMENTAL}


FAB mass spectra were obtained on a VG analytical ZAB HF instrument. All reagents and solvents were commercial and used without further purification.

Chromatography columns were prepared from Kieselgel Merck Si 60(40-63 $\mu \mathrm{m})$. TLC was performed on $250 \mu \mathrm{m}$ silica gel plates Merck containing a fluorescent indicator.

\section{Synthesis of intermediate compounds}

\section{1,3-bis-(butoxy-4-bromide)-p-tert-butylcalix[4]arene $\left(S_{2}\right)$}

Into a $250 \mathrm{~cm}^{3}$ flask containing $(3.244 \mathrm{~g}, 5.00 \mathrm{mmol})$ of tetrakis-p-tert-butylcalix[4]arene $\mathrm{S}_{1}$ and acetone $\left(50 \mathrm{~cm}^{3}\right),(1.383 \mathrm{~g}, 10.00 \mathrm{mmol})$ of $\mathrm{K}_{2} \mathrm{CO}_{3}$ were added. The mixture was stirred at room temperature for $2 \mathrm{~h}$. Then $(3.236 \mathrm{~g}, 15.00 \mathrm{mmol})$ of 1,4-butyl-dibromide in acetone $\left(50 \mathrm{~cm}^{3}\right)$ were added. The mixture was left for 4 days under reflux. After four days $5 \mathrm{~cm}^{3}$ of methanol were added. The solvents were evaporated, and the reaction mixture was dissolved in $100 \mathrm{~cm}^{3}$ of dichloromethane. After extraction with $150 \mathrm{~cm}^{3}$ of water the organic phase was dried with $\mathrm{Na}_{2} \mathrm{SO}_{4}$ and evaporated. The crude product was purified by crystallization from a $1 / 10$ dichloromethane/methanol mixture giving $(3.252 \mathrm{~g}$, $3.54 \mathrm{mmol})$ of compound $\mathbf{S}_{2}$ in $71 \%$ yield. $\mathrm{Mp}>280{ }^{\circ} \mathrm{C} .{ }^{1} \mathrm{H}$ NMR $\left(300 \mathrm{MHz}, \mathrm{CDCl}_{3}\right) \delta$ [ppm]: $0.98\left(\mathrm{~s}, 18 \mathrm{H}, \mathrm{C}-\left(\mathrm{CH}_{3}\right)_{3}\right), 1.30\left(\mathrm{~s}, 18 \mathrm{H}, \mathrm{C}-\left(\mathrm{CH}_{3}\right)_{3}\right), 2.15\left(\mathrm{qn}, 4 \mathrm{H}, \mathrm{J}=4.4, \mathrm{CH}_{2}-\mathrm{CH}_{2}-\right.$ O), 2.32 (qn, 4H, $J=4.2, \mathrm{CH}_{2}-\mathrm{CH}_{2}-\mathrm{Br}$ ), 3.32 (d, 4H, $J=13.0, \mathrm{Ar}-\mathrm{CH}_{2}-\mathrm{Ar}$ ), 3.65 (t, 4H, $J$ $\left.=6.6, \mathrm{CH}_{2}-\mathrm{CH}_{2}-\mathrm{Br}\right), 4.01\left(\mathrm{t}, 4 \mathrm{H}, J=5.8, \mathrm{CH}_{2}-\mathrm{CH}_{2}-\mathrm{O}\right), 4.25\left(\mathrm{~d}, 4 \mathrm{H}, J=13.0, \mathrm{Ar}-\mathrm{CH}_{2}-\mathrm{Ar}\right)$, 6.78 (s, 4H, Ar-H), 7.08 (s, 4H, Ar- $H$ ), 7.40 (s, 2H, OH). Anal. Calcd. for $\mathrm{C}_{52} \mathrm{H}_{70} \mathrm{O}_{4} \mathrm{Br}_{2}: \mathrm{C}$, 67.97; H, 7.68. Found: C, 68.21; H, 7.94.

\section{1,3-bis-(propoxy)-p-tert-butylcalix[4]arene $\left(S_{3}\right)$}

Into a $250 \mathrm{~cm}^{3}$ flask containing $(3.244 \mathrm{~g}, 5.00 \mathrm{mmol})$ of tetrakis-p-tert-butylcalix[4]arene $\mathrm{S}_{1}$ and acetone $\left(50 \mathrm{~cm}^{3}\right),(1.383 \mathrm{~g}, 10.00 \mathrm{mmol})$ of $\mathrm{K}_{2} \mathrm{CO}_{3}$ were added. The mixture was stirred at room temperature for $2 \mathrm{~h}$. Then $(1.845 \mathrm{~g}, 15.00 \mathrm{mmol})$ of bromopropane in acetone $\left(40 \mathrm{~cm}^{3}\right)$ were added. The mixture was left for 4 days under reflux. After four days $5 \mathrm{~cm}^{3}$ of methanol were added. The solvents were evaporated, and the reaction mixture was dissolved in $100 \mathrm{~cm}^{3}$ dichloromethane. After extraction with $150 \mathrm{~cm}^{3}$ of water the organic phase was dried with $\mathrm{Na}_{2} \mathrm{SO}_{4}$ and evaporated. The crude product was purified by crystallization from a 1/9 acetone/methanol mixture giving (2.016 g, 2.75 mmol) of pure compound $\mathrm{S}_{3}$ in $55 \%$ yield. $\mathrm{Mp}>280{ }^{\circ} \mathrm{C} .{ }^{1} \mathrm{H}$ NMR $\left(200 \mathrm{MHz}, \mathrm{CDCl}_{3}\right) \delta$ [ppm]: $1.02\left(\mathrm{~s}, 18 \mathrm{H}, \mathrm{C}-\left(\mathrm{CH}_{3}\right)_{3}\right), 1.27\left(\mathrm{t}, 6 \mathrm{H}, J=5.0, \mathrm{CH}_{3}-\mathrm{CH}_{2}-\right), 1.28\left(\mathrm{~s}, 18 \mathrm{H}, \mathrm{C}-\left(\mathrm{CH}_{3}\right)_{3}\right)$, 


\section{1,3-bis-(propoxy)-2,4-bis-(butoxy-4-bromide)-tetrakis-p-tert-butylcalix[4]arene $\left(\mathrm{S}_{4}\right)$}

Into a $250 \mathrm{~cm}^{3}$ flask containing $(2.016 \mathrm{~g}, 2.75 \mathrm{mmol})$ of $\mathbf{S}_{3}$ and DMF $\left(50 \mathrm{~cm}^{3}\right),(0.480 \mathrm{~g}$, $20.00 \mathrm{mmol}$ ) of $\mathrm{NaH}$ were added. $\mathrm{NaH}$ was washed twice with hexane before addition. The mixture was stirred at room temperature for $4 \mathrm{~h}$. After this time, (5.940 g, $27.5 \mathrm{mmol})$ of 1,4-dibromobutyl in DMF $\left(40 \mathrm{~cm}^{3}\right)$ were added. The mixture was left for 2 days at 80 $90{ }^{\circ} \mathrm{C}$. After 2 days $30 \mathrm{~cm}^{3}$ of methanol were added. The solvents were evaporated and the reaction mixture was dissolved in $100 \mathrm{~cm}^{3}$ of dichloromethane. After extraction with 250 $\mathrm{cm}^{3}$ of water the organic phase was dried with $\mathrm{Na}_{2} \mathrm{SO}_{4}$ and evaporated. The crude product was purified by crystallization from a 1/10 dichloromethane/methanol mixture giving $(1.584 \mathrm{~g}, 1.57 \mathrm{mmol})$ of compound $\mathbf{S}_{4}$ in $57 \%$ yield. Mp $170{ }^{\circ} \mathrm{C}$. ${ }^{1} \mathrm{H}$ NMR $(200 \mathrm{MHz}$, $\left.\mathrm{CDCl}_{3}\right) \delta[\mathrm{ppm}]: 1.01\left(\mathrm{t}, 6 \mathrm{H}, J=5.2, \mathrm{CH}_{3}-\mathrm{CH}_{2}-\right), 1.05\left(\mathrm{~s}, 18 \mathrm{H}, \mathrm{C}-\left(\mathrm{CH}_{3}\right)_{3}\right), 1.12(\mathrm{~s}, 18 \mathrm{H}$, $\left.\mathrm{C}-\left(\mathrm{CH}_{3}\right)_{3}\right), 1.99-2.05\left(\mathrm{~m}, 4 \mathrm{H}, \mathrm{CH}_{3}-\mathrm{CH}_{2}\right), 2.07-2.25\left(\mathrm{~m}, 8 \mathrm{H},-\mathrm{CH}_{2}-\mathrm{CH}_{2}-\mathrm{CH}_{2}-\mathrm{Br}\right), 3.13$ (d, $\left.4 \mathrm{H}, J=13.0, \mathrm{Ar}-\mathrm{CH}_{2}-\mathrm{Ar}\right), 3.51$ (t, $\left.4 \mathrm{H}, J=6.6,-\mathrm{CH}_{2}-\mathrm{Br}\right), 3.81$ (t, $4 \mathrm{H}, J=5.8, \mathrm{CH}_{3}-\mathrm{CH}_{2}-$ $\mathrm{CH}_{2}-\mathrm{O}$ ), 3.91 (t, $4 \mathrm{H}, \mathrm{J}=5.8, \mathrm{Br}_{-} \mathrm{CH}_{2}-\mathrm{CH}_{2}-\mathrm{CH}_{2}-\mathrm{CH}_{2} \mathrm{O}$ ), 4.39 (d, 4H, $J=13.0$, Ar- $\mathrm{CH}_{2}-$ Ar), 6.74 (s, 4H, Ar- $H$ ), 6.83 (s, 4H, Ar-H). Anal. Calcd for $\mathrm{C}_{58} \mathrm{H}_{82} \mathrm{O}_{4} \mathrm{Br}_{2}: \mathrm{C}, 69.45 ; \mathrm{H}$, 8.24. Found: C, 69.65; H, 8.30.

\section{Tetrakis-(butoxy-4-bromide)-tetrakis-p-tert-butylcalix[4]arene $\left(\mathrm{S}_{5}\right)$}

The suspension of p-tert-butylcalix[4]arene $\mathbf{S}_{\mathbf{1}}(1.947 \mathrm{~g}, 3.00 \mathrm{mmol})$ and $\mathrm{NaH}$ in oil washed three times with hexane $(0.700 \mathrm{~g}, 29.17 \mathrm{mmol})$ were stirred at room temperature in DMF $\left(50 \mathrm{~cm}^{3}\right)$ for $1 \mathrm{~h}$. Then 1,4-dibromobutane (12.947 $\left.\mathrm{g}, 59.06 \mathrm{mmol}\right)$ was added and the mixture was heated to $80{ }^{\circ} \mathrm{C}$. After 4 days of heating, the mixture was cooled and $\mathrm{MeOH}\left(20 \mathrm{~cm}^{3}\right)$ was added. After removing of the solvent, the residue was dissolved in dichloromethane and water and acidified with $1 \mathrm{M} \mathrm{HCl}$. The organic layer was dried over $\mathrm{Na}_{2} \mathrm{SO}_{4}$, filtered and evaporated. After precipitation from methanol the pure compound $\mathbf{S}_{5}$ ( $1.520 \mathrm{~g}, 1.28 \mathrm{mmol})$ was obtained in $43 \%$ yield. $\mathrm{Mp} 180{ }^{\circ} \mathrm{C} .{ }^{1} \mathrm{H} \mathrm{NMR}\left(300 \mathrm{MHz}, \mathrm{CDCl}_{3}\right)$ $\delta$ [ppm]: $1.09\left(\mathrm{~s}, 36 \mathrm{H},-\mathrm{C}\left(\mathrm{CH}_{3}\right)_{3}\right), 1.98-2.09\left(\mathrm{~m}, 8 \mathrm{H},-\mathrm{CH}_{2^{-}}\right), 2.16-2.23\left(\mathrm{~m}, 8 \mathrm{H},-\mathrm{CH}_{2^{-}}\right)$, 3.15 (d, 4H, $J=13.0$, Ar- $\left.\mathrm{CH}_{2}-\mathrm{Ar}\right), 3.53$ (t, $\left.8 \mathrm{H}, J=6.9,-\mathrm{CH}_{2}-\mathrm{Br}\right), 3.91$ (t, $8 \mathrm{H}, J=6.9$, - 
$\mathrm{CH}_{2}-\mathrm{O}-$ ), 4.36 (d, 4H, $\left.J=13.0, \mathrm{Ar}-\mathrm{CH}_{2}-\mathrm{Ar}\right), 6.79$, (s, 8H, Ar-H). Anal. Calcd. for $\mathrm{C}_{60} \mathrm{H}_{84} \mathrm{O}_{4} \mathrm{Br}_{4}$ : C, 60.61; H, 7.12; Found: C, 60.87; H, 7.32.

\section{Tetrakis-(4-(diphenylphosphine)-butoxy)-p-tert-butylcalix[4]arene $\left(\mathrm{S}_{6}\right)$}

Into a $100 \mathrm{~cm}^{3}$ flask containing compound $\mathrm{S}_{5}(1.510 \mathrm{~g}, 1.27 \mathrm{mmol})$ of freshly distilled THF $\left(10 \mathrm{~cm}^{3}\right),(1.282 \mathrm{~g}, 5.72 \mathrm{mmol})$ of $\mathrm{KP}(\mathrm{Ph})_{2}$ in THF $\left(15 \mathrm{~cm}^{3}\right)$ were added via a syringe. The mixture was stirred for 2 hours at room temperature, during which the colour of the reaction mixture changed from red to dark yellow. The mixture was then evaporated and extracted twice with $30 \mathrm{~cm}^{3}$ of dichloromethane. Purification of the crude product on silica column with a $3 / 7$ dichloromethane/hexane mixture as eluent gave compound $\mathbf{S}_{\mathbf{6}}$ $(0.409 \mathrm{~g}, 0.26 \mathrm{mmol})$ in $20 \%$ yield. $\mathrm{Mp} 110{ }^{\circ} \mathrm{C} .{ }^{1} \mathrm{H}$ NMR $\left(300 \mathrm{MHz}, \mathrm{CDCl}_{3}\right) \delta[\mathrm{ppm}]:$ $1.08\left(\mathrm{~s}, 36 \mathrm{H},-\mathrm{C}\left(\mathrm{CH}_{3}\right)_{3}\right), 1.42-1.63\left(\mathrm{~m}, 8 \mathrm{H},-\mathrm{CH}_{2}-\mathrm{CH}_{2}-\mathrm{P}\right), 2.00-2.18\left(\mathrm{~m}, 8 \mathrm{H},-\mathrm{CH}_{2}-\mathrm{CH}_{2}-\right.$ O), 2.00-2.18 (m, 8H, -CH $\left.H_{2}-\mathrm{P}\right), 3.05$ (d, 4H, $J=12.9$, Ar-CH $\left.-\mathrm{Ar}\right), 3.81$ (t, 8H, $J=5.4$, $\mathrm{CH}_{2}-\mathrm{CH}_{2}-\mathrm{O}$ ), 4.29 (d, 4H, $J=12.9$, Ar- $\left.\mathrm{CH}_{2}-\mathrm{Ar}\right), 6.75$ (s, 8H, Ar- $\left.H\right), 7.20-7.46(\mathrm{~m}, 40 \mathrm{H}$, P-Ar-H). ${ }^{31} \mathrm{P}$ NMR (400 MHz, $\left.\mathrm{CDCl}_{3}\right) \delta$ [ppm]: -14.91 [P]. m/z (MALDI) 1610.88 (M + H) ${ }^{+}$. Anal. Calcd. for $\mathrm{C}_{108} \mathrm{H}_{124} \mathrm{O}_{4} \mathrm{P}_{4}$ : C, 80.57; H, 7.76; Found: C, 80.73; H, 7.87.

\section{Synthesis of phosphonium ligands}

\section{1,3-bis-(4-triphenylphosphonium-butoxy)-p-tert-butylcalix[4]arene dibromide (1)}

Into a $100 \mathrm{~cm}^{3}$ flask containing $\mathbf{S}_{2}(1.184 \mathrm{~g}, 2.00 \mathrm{mmol})$ in chloroform $\left(30 \mathrm{~cm}^{3}\right),(5.248 \mathrm{~g}$, $20.00 \mathrm{mmol})$ of triphenylphosphine in chloroform $\left(20 \mathrm{~cm}^{3}\right)$ were added. After 6 days under reflux, the mixture was cooled and the solvent evaporated. The residue was dissolved in dichloromethane. The excess of triphenylphosphine was precipitated from methanol and filtered out. The filtrate was evaporated. The pure product $\mathbf{1}(2.179 \mathrm{~g}, 1.51$ mmol) was obtained by precipitation from a $1 / 9$ dichloromethane/hexane mixture, as a white-light green powder in $76 \%$ yield: $\mathrm{Mp}>280{ }^{\circ} \mathrm{C} .{ }^{1} \mathrm{H} \mathrm{NMR}\left(300 \mathrm{MHz}, \mathrm{CDCl}_{3}\right) \delta$ [ppm]: $0.99\left(\mathrm{~s}, 18 \mathrm{H},-\mathrm{C}\left(\mathrm{CH}_{3}\right)_{3}\right), 1.28\left(\mathrm{~s}, 18 \mathrm{H},-\mathrm{C}\left(\mathrm{CH}_{3}\right)_{3}\right), 2.05-2.29\left(\mathrm{~m}, 8 \mathrm{H}, \mathrm{CH}_{2}-\mathrm{CH}_{2}-\right.$ $\mathrm{CH}_{2}-\mathrm{CH}_{2}-\mathrm{O}$ ), 3.19 (d, $4 \mathrm{H}, \mathrm{J}=12.8, \mathrm{Ar}-\mathrm{CH}_{2}-\mathrm{Ar}$ ), 3.80-4.00 (m, 4H, $\left.-\mathrm{CH}_{2}-\mathrm{O}\right), 3.90$ (d, 4H, $\left.J=12.8, \mathrm{Ar}-\mathrm{CH}_{2}-\mathrm{Ar}\right), 3.90-4.08\left(\mathrm{~m}, 4 \mathrm{H},-\mathrm{CH}_{2}-\mathrm{P}\right), 6.79$ (s, 4H, Ar- $H$ ), 6.99 (s, 4H, Ar- $H$ ), 7.49 (s, 2H, OH), 7.54 - 7.64 (m, 12H, P-Ar-H meta), 7.65-7.74 (m, 6H, P-Ar-H para), 7.80-7.93 (m, 12H, P-Ar-H ortho). ${ }^{31} \mathrm{P}$ NMR (400 MHz, $\left.\mathrm{CDCl}_{3}\right) \delta[\mathrm{ppm}]: 25.80 . \mathrm{m} / \mathrm{z}$ $\left(\mathrm{FAB}^{+}\right) 721.7(\mathrm{M}+2 \mathrm{H})^{2+} ; \mathrm{m} / \mathrm{z}(\mathrm{MALDI}) 1361.7(\mathrm{M}-\mathrm{Br})^{+}$. Anal. Calcd for $\mathrm{C}_{88} \mathrm{H}_{100} \mathrm{O}_{4} \mathrm{P}_{2} \mathrm{Br}_{2}$ : C, 73.22; H, 6.98. Found: C, 73.46; H, 7.20. 


\section{1,3-bis-(4-(P,P-diphenyl-P-methylphosphonium)-butoxy)-p-tert-butylcalix[4]arene dibromide (2)}

Compound 2 was prepared following the same procedure as for compound $\mathbf{1}$ with $\mathbf{S}_{2}$ (2.753 g, $3.00 \mathrm{mmol})$ and diphenylmethylphosphine (6.006 g, $30.00 \mathrm{mmol})$ in $69 \%$ yield. $\mathrm{Mp}>280{ }^{\circ} \mathrm{C} .{ }^{1} \mathrm{H}$ NMR $\left(300 \mathrm{MHz}, \mathrm{CDCl}_{3}\right) \delta[\mathrm{ppm}]: 0.96\left(\mathrm{~s}, 18 \mathrm{H},-\mathrm{C}\left(\mathrm{CH}_{3}\right)_{3}\right), 1.29(\mathrm{~s}, 18 \mathrm{H}$, $\left.-\mathrm{C}\left(\mathrm{CH}_{3}\right)_{3}\right), 1.91-2.10\left(\mathrm{~m}, 4 \mathrm{H},-\mathrm{CH}_{2}-\mathrm{CH}_{2}-\mathrm{P}\right), 2.11-2.27\left(\mathrm{~m}, 4 \mathrm{H},-\mathrm{CH}_{2}-\mathrm{CH}_{2}-\mathrm{O}\right), 2.99(\mathrm{~d}, 6 \mathrm{H}$, $\left.J=13.8, \mathrm{CH}_{3}-\mathrm{P}\right), 3.23\left(\mathrm{~d}, 4 \mathrm{H}, J=13.5, \mathrm{Ar}-\mathrm{CH}_{2}-\mathrm{Ar}\right), 3.58-3.75\left(\mathrm{~m}, 4 \mathrm{H},-\mathrm{CH}_{2}-\mathrm{P}\right), 3.90$ (t, $\left.4 \mathrm{H}, J=5.3,-\mathrm{CH}_{2} \mathrm{O}\right), 4.02\left(\mathrm{~d}, 4 \mathrm{H}, J=13.5, \mathrm{Ar}-\mathrm{CH}_{2}-\mathrm{Ar}\right), 6.76(\mathrm{~s}, 4 \mathrm{H}, \mathrm{Ar}-\mathrm{H}), 7.01(\mathrm{~s}, 4 \mathrm{H}$, Ar-H), $7.31(\mathrm{~s}, 2 \mathrm{H}, \mathrm{OH}), 7.48-7.59$ (m, $8 \mathrm{H}, \mathrm{P}-\mathrm{Ar}-H$ meta), 7.61-7.70 (m, 4H, P-Ar-H para), 7.90-8.06 (m, 8H, P-Ar-H ortho). ${ }^{31} \mathrm{P}$ NMR (400 MHz, $\left.\mathrm{CDCl}_{3}\right) \delta[\mathrm{ppm}]: 25.96 . \mathrm{m} / \mathrm{z}$ (MALDI) $1239.58(\mathrm{M}-\mathrm{Br})^{+}$. Anal. Calcd. for $\mathrm{C}_{78} \mathrm{H}_{96} \mathrm{O}_{4} \mathrm{P}_{2} \mathrm{Br}_{2}: \mathrm{C}, 71.01 ; \mathrm{H}, 7.33$. Found: C, 71.21; H, 7.52.

\section{1,3-bis-(4-triphenylphosphonium-butoxy)-2,4-bis-propoxy-p-tert-butyl-calix[4]arene dibromide (3)}

Into a $100 \mathrm{~cm}^{3}$ flask containing $\mathbf{S}_{4}(2.012 \mathrm{~g}, 2.00 \mathrm{mmol})$ in chloroform $\left(30 \mathrm{~cm}^{3}\right), 5.248 \mathrm{~g}$ of triphenylphosphine $(20.00 \mathrm{mmol})$ in chloroform $\left(20 \mathrm{~cm}^{3}\right)$ were added and left for 6 days under reflux. After that time the mixture was cooled and the solvent evaporated. The residue was dissolved in dichloromethane. The excess of triphenylphosphine was precipitated from methanol and filtered off. The filtrate was evaporated. The pure product $3(2.179 \mathrm{~g}, 1.51 \mathrm{mmol})$ was obtained by precipitation from a $1 / 9$ dichloromethane/hexane mixture, as a white-light green powder in $76 \%$ yield. Mp $120{ }^{\circ} \mathrm{C} .{ }^{1} \mathrm{H}$ NMR $(300 \mathrm{MHz}$, $\left.\mathrm{CDCl}_{3}\right) \delta[\mathrm{ppm}]: 0.86\left(\mathrm{t}, 6 \mathrm{H}, J=5.3, \mathrm{CH}_{3}-\mathrm{CH}_{2}-\mathrm{CH}_{2}-\mathrm{O}\right), 0.99\left(\mathrm{~s}, 18 \mathrm{H},-\mathrm{C}\left(\mathrm{CH}_{3}\right)_{3}\right), 1.11(\mathrm{~s}$, $\left.18 \mathrm{H},-\mathrm{C}\left(\mathrm{CH}_{3}\right)_{3}\right), 1.65-1.87\left(\mathrm{~m}, 4 \mathrm{H}, \mathrm{CH}_{3}-\mathrm{CH}_{2}-\mathrm{CH}_{2}-\mathrm{O}\right), 1.77-1.94\left(\mathrm{~m}, 4 \mathrm{H},-\mathrm{CH}_{2}-\mathrm{CH}_{2}-\mathrm{P}\right)$, 2.28-2.43 (m, 4H, $\left.-\mathrm{CH}_{2}-\mathrm{CH}_{2}-\mathrm{O}\right), 2.95$ (d, 4H, J=13.0, Ar- $\left.\mathrm{CH}_{2}-\mathrm{Ar}\right), 3.63(\mathrm{t}, 4 \mathrm{H}, J=5.9$, $\left.\mathrm{CH}_{3}-\mathrm{CH}_{2}-\mathrm{CH}_{2}-\mathrm{O}\right), 3.85-4.00\left(\mathrm{~m}, 4 \mathrm{H},-\mathrm{CH}_{2}-\mathrm{P}\right), 3.85-4.00\left(\mathrm{~m}, 4 \mathrm{H},-\mathrm{CH}_{2}-\mathrm{O}\right), 4.18(\mathrm{~d}, 4 \mathrm{H}, \mathrm{J}$ $\left.=13.0, \mathrm{Ar}-\mathrm{CH}_{2}-\mathrm{Ar}\right), 6.61(\mathrm{~s}, 4 \mathrm{H}, \mathrm{Ar}-H), 6.76(\mathrm{~s}, 4 \mathrm{H}, \mathrm{Ar}-H), 7.60-7.92(\mathrm{~m}, 30 \mathrm{H}, \mathrm{P}-\mathrm{Ar}-\mathrm{H}$ ortho, meta, para). ${ }^{31} \mathrm{P}$ NMR (400 MHz, $\left.\mathrm{CDCl}_{3}\right) \delta[\mathrm{ppm}]: 25.84 . \mathrm{m} / \mathrm{z}$ (MALDI) 1447.6 (M $-\mathrm{Br})^{+}$. Anal. Calcd. for $\mathrm{C}_{94} \mathrm{H}_{112} \mathrm{O}_{4} \mathrm{P}_{2} \mathrm{Br}_{2}$ : C, 73.91; H, 7.39. Found: C, 73.67; H, 7.66.

\section{1,3-bis-propoxy-2,4-bis-(4-(P,P-diphenyl-P-methylphosphonium)-butoxy)-p-tert- butylcalix[4]arene dibromide (4)}

Compound 4 was obtained according to the same procedure as for $\mathbf{3}$ with $\mathbf{S}_{\mathbf{4}}(3.050 \mathrm{~g}, 3.04$ 
mmol) and diphenylmethylphosphine (6.006 g, $30.00 \mathrm{mmol})$ a white powder in $64 \%$ yield. Mp $148{ }^{\circ} \mathrm{C} .{ }^{1} \mathrm{H}$ NMR $\left(300 \mathrm{MHz}, \mathrm{CDCl}_{3}\right) \delta[\mathrm{ppm}]: 0.79\left(\mathrm{~s}, 18 \mathrm{H},-\mathrm{C}\left(\mathrm{CH}_{3}\right)_{3}\right), 1.03(\mathrm{t}$, $\left.6 \mathrm{H}, \mathrm{J}=6.9, \mathrm{CH}_{3}-\mathrm{CH}_{2}-\mathrm{CH}_{2}-\mathrm{O}\right), 1.31\left(\mathrm{~s}, 18 \mathrm{H},-\mathrm{C}\left(\mathrm{CH}_{3}\right)_{3}\right), 1.55-1.75\left(\mathrm{~m}, 4 \mathrm{H},-\mathrm{CH}_{2}-\mathrm{CH}_{2}-\mathrm{P}\right)$, 1.88-2.04 (m, 4H, $\mathrm{CH}_{3}-\mathrm{CH}_{2}-\mathrm{CH}_{2}-\mathrm{O}$ ), 2.40-2.58 (m, 4H, $\mathrm{CH}_{2}-\mathrm{CH}_{2}-\mathrm{CH}_{2}-\mathrm{O}$ ), 3.05 (d, 4H, J $\left.=12.5, \mathrm{Ar}-\mathrm{CH}_{2}-\mathrm{Ar}\right), 3.23\left(\mathrm{~d}, 6 \mathrm{H}, \mathrm{J}=14.3, \mathrm{CH}_{3}-\mathrm{P}\right), 3.62-3.80\left(\mathrm{~m}, 8 \mathrm{H},-\mathrm{CH}_{2}-\mathrm{P}\right.$ and $-\mathrm{CH}_{2}-$ O), 3.88 (t, 4H, $\left.J=5.9,-\mathrm{CH}_{2}-\mathrm{CH}_{2}-\mathrm{CH}_{2}-\mathrm{O}\right), 4.32$ (d, $4 \mathrm{H}, J=12.5, \mathrm{Ar}-\mathrm{CH}_{2}-\mathrm{Ar}$ ), 6.43 (s, 4H, Ar-H), 7.08 (s, 4H, Ar-H), 7.60-7.80 (m, 12H, P-Ar-H meta, para), 8.01-8.13 (m, 8H, P-Ar-H ortho). ${ }^{31} \mathrm{P}$ NMR (400 MHz, $\left.\mathrm{CDCl}_{3}\right) \delta[\mathrm{ppm}]: 25.98 . \mathrm{m} / \mathrm{z}$ (MALDI) 1323.7 (M Br) ${ }^{+}$. Anal. Calcd. for $\mathrm{C}_{84} \mathrm{H}_{108} \mathrm{O}_{4} \mathrm{P}_{2} \mathrm{Br}_{2}$ : C, 71.88; H, 7.76. Found: C, 71.99; H, 7.85.

\section{Tetrakis-(4-triphenylphosphonium-butoxy)-tetrakis-p-tert-butylcalix[4]arene tetrabromide (5)}

Compound $\mathbf{S}_{5}(1.184 \mathrm{~g}, 1.00 \mathrm{mmol})$ was dissolved in chloroform $\left(30 \mathrm{~cm}^{3}\right)$. After a few minutes of stirring triphenylphosphine $(5.248 \mathrm{~g}, 20.00 \mathrm{mmol})$ and chloroform $\left(20 \mathrm{~cm}^{3}\right)$ were added. After 6 days of refluxing the mixture was cooled and solvent was evaporated. The residue was dissolved in dichloromethane. The excess of triphenylphosphine was precipitated from methanol and filtered. The organic layer was evaporated. Chromatography on a silica column with 90:10 dichloromethane: methanol mixture as eluent gave compound $5(0.67 \mathrm{~g}, 0.30 \mathrm{mmol})$ in $30 \%$ yield. Mp $132{ }^{\circ} \mathrm{C}$. ${ }^{1} \mathrm{H}$ NMR (300 $\left.\mathrm{MHz} ; \mathrm{CDCl}_{3}\right) \delta[\mathrm{ppm}]: 1.02\left(\mathrm{~s}, 36 \mathrm{H},-\mathrm{C}\left(\mathrm{CH}_{3}\right)_{3}\right), 1.56-1.72\left(\mathrm{~m}, 8 \mathrm{H},-\mathrm{CH}_{2^{-}}\right), 2.24-2.41(\mathrm{~m}$, $\left.8 \mathrm{H},-\mathrm{CH}_{2}-\right), 2.91\left(\mathrm{~d}, 4 \mathrm{H}, \mathrm{J}=13.0, \mathrm{Ar}-\mathrm{CH}_{2}-\mathrm{Ar}\right), 3.78-4.01\left(\mathrm{~m}, 16 \mathrm{H},-\mathrm{CH}_{2}-\mathrm{P}\right.$ and Ar-O$\mathrm{CH}_{2}$ ), 4.23 (d, 4H, $\left.J=13.0, \mathrm{Ar}-\mathrm{CH}_{2}-\mathrm{Ar}\right), 6.63$ (s, 8H, Ar- $H$ ), 7.59-7.71- (m, 36H, P-Ar-H, meta, para), 7.76-7.88 (m, 24H, P-Ar-H, ortho). ${ }^{31} \mathrm{P}$ NMR $\left(400 \mathrm{MHz}, \mathrm{CDCl}_{3}\right) \delta[\mathrm{ppm}]$ : 25.76. $\mathrm{m} / \mathrm{z}\left(\mathrm{FAB}^{+}\right) 479.5(\mathrm{M})^{4+} ; \mathrm{m} / \mathrm{z}(\mathrm{MALDI}) 2157.7(\mathrm{M}-\mathrm{Br})^{+}$. Anal. Calcd. for $\mathrm{C}_{132} \mathrm{H}_{144} \mathrm{O}_{4} \mathrm{P}_{4} \mathrm{Br}_{4}$ : C, 70.84; H, 6.49. Found: C, 70.97; H, 6.69.

\section{Tetrakis-(4-triphenylphosphonium-butoxy)-p-tert-butylcalix[4]arene tetraperchlorate} (5a)

In a $10 \mathrm{~cm}^{3}$ flask $5(0.100 \mathrm{~g}, 0.045 \mathrm{mmol})$ was dissolved in acetonitrile $\left(1 \mathrm{~cm}^{3}\right) .(0.050 \mathrm{~g}$, $0.241 \mathrm{mmol})$ of $\mathrm{AgClO}_{4}$ in acetonitrile $\left(1 \mathrm{~cm}^{3}\right)$ were added dropwise to the ligand solution. After 24 hours of stirring at room temperature, the precipitate of $\mathrm{AgBr}$ was filtered off. The filtrate was evaporated to give compound $5 \mathbf{a}(0.088 \mathrm{~g}, 0.038 \mathrm{mmol})$ in 84 $\%$ yield. $\mathrm{Mp}>120{ }^{\circ} \mathrm{C}$ decomposition. ${ }^{1} \mathrm{H}$ NMR $\left(300 \mathrm{MHz}, \mathrm{CDCl}_{3}\right) \delta[\mathrm{ppm}]: 1.07(\mathrm{~s}, 36 \mathrm{H}$, $\left.-\mathrm{C}\left(\mathrm{CH}_{3}\right)_{3}\right), 1.70-1.85\left(\mathrm{~m}, 8 \mathrm{H},-\mathrm{CH}_{2}-\mathrm{CH}_{2}-\mathrm{P}\right), 2.45-2.53\left(\mathrm{~m}, 8 \mathrm{H},-\mathrm{CH}_{2}-\mathrm{CH}_{2}-\mathrm{O}\right), 2.95(\mathrm{~d}, 4 \mathrm{H}$, 
Tetrakis-(4-triphenylphosphonium-butoxy)-tetrakis-p-tert-butylcalix[4]arene tetrahexafluorophosphate (5b)

Compound 5 (0.100 g, 0.045 mmoles) was dissolved in acetonitrile $\left(1 \mathrm{~cm}^{3}\right)$. Then $(0.062$ g, $0.245 \mathrm{mmol}) \mathrm{AgPF}_{6}$ were dissolved in acetonitrile and added dropwise to the ligand solution. After $24 \mathrm{~h}$ the precipitate of $\mathrm{NaBr}$ was removed and the solution was evaporated. Compound $5 \mathbf{b}$ (0.090 g, 0.036 mmole) was obtained in $80 \%$ yield. Mp $128{ }^{\circ} \mathrm{C} .{ }^{1} \mathrm{H}$ NMR $\left(500 \mathrm{MHz} ; \mathrm{CDCl}_{3}\right) \delta[\mathrm{ppm}]: 1.12\left(\mathrm{~s}, 36 \mathrm{H},-\mathrm{C}\left(\mathrm{CH}_{3}\right)_{3}\right), 1.65-1.81\left(\mathrm{~m}, 8 \mathrm{H},-\mathrm{CH}_{2^{-}}\right), 2.38-2.49$ (m, 8H, $\left.-\mathrm{CH}_{2^{-}}\right), 3.25-3.38\left(\mathrm{~m}, 8 \mathrm{H},-\mathrm{CH}_{2}-\mathrm{P}\right), 3.47$ (d, 4H, $\left.J=13.0, \mathrm{Ar}-\mathrm{CH}_{2}-\mathrm{Ar}\right), 4.15-4.24$ (m, 8H, Ar-O-CH $H_{2}$, 4.47 (d, 4H, $\left.J=13.0, A r-C_{2}-\mathrm{Ar}\right), 7.04$ (s, 8H, Ar- $H$ ), 7.66-7.70 (m, $60 \mathrm{H}, \mathrm{P}-\mathrm{Ar}-H) .{ }^{31} \mathrm{P}$ NMR $\left(400 \mathrm{MHz}, \mathrm{CDCl}_{3}\right) \delta[\mathrm{ppm}]: 24.72\left[\mathrm{P}^{+}\right],-143.39\left[\mathrm{PF}_{6}\right] . \mathrm{m} / \mathrm{z}$ (MALDI) $1447.60\left(\mathrm{M}-\mathrm{PF}_{6}\right)^{+}$. Anal. Calcd. for $\mathrm{C}_{132} \mathrm{H}_{144} \mathrm{O}_{4} \mathrm{P}_{4}\left(\mathrm{PF}_{6}\right)_{4}: \mathrm{C}, 63.46 ; \mathrm{H}, 5.81$. Found: C, 63.30; H, 5.78.

\section{Tetrakis-(4-(P,P-diphenyl-P-methylphosphonium)-butoxy)-p-tert-butylcalix[4]arene} tetrabromide (6)

Into a $100 \mathrm{~cm}^{3}$ flask containing S5 $(1.184 \mathrm{~g}, 1.00 \mathrm{mmol})$ dissolved in chloroform (30 $\left.\mathrm{cm}^{3}\right)$, (4.004 g, $\left.20.00 \mathrm{mmol}\right)$ diphenylmethylphosphine in chloroform $\left(20 \mathrm{~cm}^{3}\right)$ were added. After 6 days under reflux the mixture was cooled and the solvent was evaporated. The product was purified by precipitation from a 1/9 dichloromethane/hexane mixture to give compound $6(1.190 \mathrm{~g}, 0.59 \mathrm{mmol})$ in $59 \%$ yield. Mp $160{ }^{\circ} \mathrm{C} .{ }^{1} \mathrm{H}$ NMR $(300 \mathrm{MHz}$; $\left.\mathrm{CDCl}_{3}\right) \delta[\mathrm{ppm}]: 1.03\left(\mathrm{~s}, 36 \mathrm{H},-\mathrm{C}\left(\mathrm{CH}_{3}\right)_{3}\right), 1.55-1.75\left(\mathrm{~m}, 8 \mathrm{H},-\mathrm{CH}_{2}-\mathrm{CH}_{2}-\mathrm{P}\right), 2.30-2.48(\mathrm{~m}$, $8 \mathrm{H},-\mathrm{CH}_{2}-\mathrm{CH}_{2}-\mathrm{O}$ ), 2.91 (d, $12 \mathrm{H}, J=13.5, \mathrm{CH}_{3}-\mathrm{P}$ ), 3.03 (d, 4H, J = 12.8, Ar-CH $-\mathrm{Ar}$ ), 3.70-3.87 (m, 16H, - $\mathrm{CH}_{2}-\mathrm{P}$ and $\left.\mathrm{CH}_{2}-\mathrm{O}\right), 4.26\left(\mathrm{~d}, 4 \mathrm{H}, J=12.8, \mathrm{Ar}-\mathrm{CH}_{2}-\mathrm{Ar}\right), 6.71(\mathrm{~s}, 8 \mathrm{H}$, Ar-H), 7.54-7.78 (m, 24H, P-Ar-H meta, para), 8.02-8.17 (m, 16H, P-Ar-H ortho). ${ }^{31} \mathrm{P}$ NMR (400 MHz, $\left.\mathrm{CDCl}_{3}\right) \delta[\mathrm{ppm}]:$ 25.98. m/z (MALDI) 1909.68 (M - Br) ${ }^{+}$. Anal. Calcd. for $\mathrm{C}_{112} \mathrm{H}_{136} \mathrm{O}_{4} \mathrm{P}_{4} \mathrm{Br}_{4}$ : C, 67.61; H, 6.89. Found: C, 68.59; H, 7.10.

\section{Tetrakis-(4-(P,P-diphenyl-P-methylphosphonium)-butoxy)-p-tert-butylcalix[4]arene}




\section{tetraperchlorate (6a)}

Compound 6a was obtained according to the same procedure as 5a with $6(0.100 \mathrm{~g}, 0.05$ mmol) and $\mathrm{AgClO}_{4}(0.050 \mathrm{~g}, 0.24 \mathrm{mmol})$ in $90 \%$ yield. $\mathrm{Mp}>120{ }^{\circ} \mathrm{C}$ decomposition. ${ }^{1} \mathrm{H}$ NMR (300 MHz, $\left.\mathrm{CDCl}_{3}\right) \delta[\mathrm{ppm}]: 1.09\left(\mathrm{~s}, 36 \mathrm{H},-\mathrm{C}\left(\mathrm{CH}_{3}\right)_{3}\right), 1.65-1.80\left(\mathrm{~m}, 8 \mathrm{H},-\mathrm{CH}_{2}-\mathrm{CH}_{2}-\right.$ P), 2.35-2.50 (m, 8H, $\left.-\mathrm{CH}_{2}-\mathrm{CH}_{2}-\mathrm{O}\right), 2.50\left(\mathrm{~d}, 12 \mathrm{H}, \mathrm{J}=12.0, \mathrm{CH}_{3}-\mathrm{P}\right), 3.18-3.28(\mathrm{~m}, 8 \mathrm{H}$, $\left.\mathrm{CH}_{2}-\mathrm{P}\right), 3.38$ (d, 4H, $\left.\mathrm{J}=12.8, \mathrm{Ar}-\mathrm{CH}_{2}-\mathrm{Ar}\right), 4.15-4.28\left(\mathrm{~m}, 8 \mathrm{H},-\mathrm{CH}_{2}-\mathrm{O}\right), 4.45$ (d, $4 \mathrm{H}, J=$ 12.8, Ar- $\mathrm{CH}_{2}$-Ar), 6.97 (s, 8H, Ar-H), 7.60-7.87 (m, 40H, P-Ar-H, ortho, meta, para). ${ }^{31} \mathrm{P}$ NMR (400 MHz, $\left.\mathrm{CDCl}_{3}\right) \delta[\mathrm{ppm}]:$ 24.77. m/z (MALDI) $1966.24\left(\mathrm{M}-\mathrm{ClO}_{4}\right)^{+}$. Anal. Calcd. for $\mathrm{C}_{112} \mathrm{H}_{136} \mathrm{O}_{4} \mathrm{P}_{4}\left(\mathrm{ClO}_{4}\right)_{4}$ : C, 65.05; H, 6.63. Found: C, 65.13; H, 6.72.

\section{Tetrakis-(4-(P,P-diphenyl-P-methylphosphonium)-butoxy)-p-tert-butylcalix[4]arene}

\section{tetrahexafluorophosphate (6b)}

Compound $\mathbf{6 b}$ was obtained according to the same procedure as for $5 \mathbf{b}$ with $\mathbf{6}(0.100 \mathrm{~g}$, $0.05 \mathrm{mmol})$ and $\mathrm{AgPF}_{6}(0.069 \mathrm{~g}, 0.27 \mathrm{mmol})$ in $80 \%$ yield. Mp $155{ }^{\circ} \mathrm{C} .{ }^{1} \mathrm{H}$ NMR (300 $\left.\mathrm{MHz}, \mathrm{CDCl}_{3}\right) \delta[\mathrm{ppm}]: 1.11\left(\mathrm{~s}, 36 \mathrm{H},-\mathrm{C}\left(\mathrm{CH}_{3}\right)_{3}\right), 1.55-1.70\left(\mathrm{~m}, 8 \mathrm{H},-\mathrm{CH}_{2}-\mathrm{CH}_{2}-\mathrm{P}\right), 2.24-$ $2.38\left(\mathrm{~m}, 8 \mathrm{H},-\mathrm{CH}_{2}-\mathrm{CH}_{2}-\mathrm{O}\right), 2.42\left(\mathrm{~d}, 12 \mathrm{H}, \mathrm{J}=12.0, \mathrm{CH}_{3}-\mathrm{P}\right), 2.95-3.10\left(\mathrm{~m}, 8 \mathrm{H},-\mathrm{CH}_{2}-\mathrm{P}\right)$, 3.39 (d, $4 \mathrm{H}, J=12.8$, Ar- $\mathrm{CH}_{2}-\mathrm{Ar}$ ), 4.05-4.20 (m, 8H, $\left.-\mathrm{CH}_{2}-\mathrm{O}\right), 4.42$ (d, 4H, $J=12.8$, Ar$\mathrm{CH}_{2}$-Ar), 7.00 (s, 8H, Ar-H), 7.58-7.80 (m, 40H, P-Ar-H, ortho, meta, para). ${ }^{31} \mathrm{P}$ NMR $\left(400 \mathrm{MHz}, \mathrm{CDCl}_{3}\right) \delta[\mathrm{ppm}]: 24.50\left[\mathrm{P}^{+}\right],-143.07\left[\mathrm{PF}_{6}\right], \mathrm{m} / \mathrm{z}(\mathrm{MALDI}) 2104.80\left(\mathrm{M}-\mathrm{PF}_{6}\right)^{+}$. Anal. Calcd. for $\mathrm{C}_{112} \mathrm{H}_{136} \mathrm{O}_{4} \mathrm{P}_{4}\left(\mathrm{PF}_{6}\right)_{4}$ : C, 59.79; H, 6.09. Found: C, 59.87; H, 6.03.

\section{Protonated tetrakis-(4-(P,P-diphenyl-phosphine)-butoxy)-p-tert-butylcalix[4]arene} tetrabromide (7)

Into a $50 \mathrm{~cm}^{3}$ flask containing $\mathbf{S}_{\mathbf{6}}(1.510 \mathrm{~g}, 0.94 \mathrm{mmol})$ in dichloromethane $\left(10 \mathrm{~cm}^{3}\right), 5$ $\mathrm{cm}^{3}$ of $\mathrm{HBr}$ (solution of $33 \%$ wt. in glacial acetic acid) in dichloromethane $\left(15 \mathrm{~cm}^{3}\right)$ were added. The mixture was left stirred for 24 hours at room temperature. The mixture was then evaporated to give the pure product $7(0.954 \mathrm{~g}, 0.49 \mathrm{mmol})$ in $52 \%$ yield. Mp 175 ${ }^{\circ} \mathrm{C} .{ }^{1} \mathrm{H}$ NMR $\left(300 \mathrm{MHz}, \mathrm{CDCl}_{3}\right) \delta[\mathrm{ppm}]: 1.22\left(\mathrm{~s}, 36 \mathrm{H},-\mathrm{C}\left(\mathrm{CH}_{3}\right)_{3}\right), 1.80-2.15(\mathrm{~m}, 16 \mathrm{H}, \mathrm{O}-$ $\mathrm{CH}_{2}-\mathrm{CH}_{2}-\mathrm{CH}_{2}-\mathrm{CH}_{2}-\mathrm{P}$ ), 2.92-3.10 (m, 4H, Ar- $\mathrm{CH}_{2}-\mathrm{Ar}$ ), 3.18-3.30 (m, 8H, $\mathrm{CH}_{2}-\mathrm{O}$ ), 3.40$3.55\left(\mathrm{~m}, 8 \mathrm{H},-\mathrm{CH}_{2}-\mathrm{P}\right), 4.05-4.27$ (m, 4H, Ar- $\mathrm{CH}_{2}-\mathrm{Ar}$ ), 7.05 (s, 8H, Ar- $\mathrm{H}$ ), 7.60-8.05 (m, 40H, P-Ar-H, ortho, meta, para), 10.30-10.40 (m, 4H, H-P). ${ }^{31} \mathrm{P}$ NMR (400 MHz, $\mathrm{CDCl}_{3}$ ) $\delta$ [ppm]: $46.32\left[\mathrm{P}^{+}\right] . \mathrm{m} / \mathrm{z}$ (MALDI) $1849.04(\mathrm{M}-\mathrm{Br})^{+}$. Anal. Calcd. for $\mathrm{C}_{108} \mathrm{H}_{128} \mathrm{O}_{4} \mathrm{P}_{4} \mathrm{Br}_{4}$ : C, 67.08; H, 6.67. Found: C, 66.84; H, 6.92. 


\section{Microcalorimetric studies}

Microcalorimetric titrations were performed using a 2277 Thermal Activity Monitor Microcalorimeter (Thermometric). Titration were carried out at $25{ }^{\circ} \mathrm{C}$ on $2.7 \mathrm{~cm}^{3}$ of $10^{-5}$ to $5 \times 10^{-4} \mathrm{M}$ solutions of the ligand in acetonitrile using a glass cell of $4 \mathrm{~cm}^{3}$. The heat changes were measured after injection of $15 \times 15 \mu \mathrm{L}$ of $10^{-3}$ and $10^{-2} \mathrm{M} \mathrm{LiClO}_{4}, \mathrm{LiBr}, \mathrm{NaClO}_{4}, \mathrm{NaPF}_{6}$, or $\mathrm{Et}_{4} \mathrm{NClO}_{4}$ solutions in the same solvent. Chemical calibration was made by determination of the complexation enthalpy of $\mathrm{Ba}^{2+}$ with $18 \mathrm{C} 6$ in water or of $\mathrm{Rb}^{+}$with $18 \mathrm{C} 6$ in methanol, as recommended [37]. Values of the stability constants $(\beta)$ and of the enthalpies of complexation $(\Delta H)$ were refined simultaneously from these data using the ligand binding analysis program DIGITAM version 4.1 [38] and after correction for the heat of dilution determined in separate experiments by adding the salt solutions to $2.7 \mathrm{~cm}^{3}$ of pure solvent. The values of the corresponding entropies of complexation $(\Delta S)$ were then derived from the expressions $\Delta G=-R T \ln \beta$ and $\Delta G=\Delta H-T \Delta S$.

\section{Ion selective electrodes}

THF was dried and freshly distilled before used for the preparation of the ion selective membranes. PVC (high molecular weight poly(vinyl chloride), 2-nitrophenyl octyl ether (o-NPOE), bis-(2-ethylhexyl)sebacate (BEHS), (2-morpholino)ethanesulfonic acid monohydrate (MES) were from Fluka Selectophore. The $\mathrm{LiClO}_{4}, \mathrm{CsClO}_{4}$ and sodium salts: $\mathrm{Cl}^{-}, \mathrm{Br}^{-}, \mathrm{I}^{-}, \mathrm{ClO}_{4}{ }^{-}, \mathrm{SCN}^{-}, \mathrm{NO}_{3}{ }^{-}, \mathrm{SO}_{4}{ }^{2-}, \mathrm{CO}_{3}{ }^{2-}, \mathrm{HPO}_{4}{ }^{2-}, \mathrm{PO}_{4}{ }^{3-}, \mathrm{Cr}_{2} \mathrm{O}_{7}{ }^{2-}$, citrate, acetate, benzoate, and oxalate were of p.a. grade. All aqueous salt solutions were prepared with demineralised water (conductivity $<1.0 \mu \mathrm{S} / \mathrm{cm}$ ).

The membranes were composed of $4 \mathrm{mg}$ of ionophores $\mathbf{1}-\mathbf{6}$ and $\mathbf{8}, 60 \mathrm{mg}$ of PVC and 120 $\mathrm{mg}$ of plasticizer. All the components were dissolved in $1.5 \mathrm{~cm}^{3}$ of dried, freshly distilled 
THF and the solutions were poured into glass rings of $24 \mathrm{~mm}$ in diameter. The solutions were left for $24 \mathrm{~h}$ for slow solvent evaporation giving the mother membranes of thickness about $0.1 \mathrm{~mm}$. Several membranes of $7 \mathrm{~mm}$ diameter were cut from each mother membrane and were incorporated into the $\mathrm{Ag} / \mathrm{AgCl}$ electrodes bodies of IS 561 type (Moeller S.A., Zurich). The two plasticizers BEHS and $o$-NPOE were used for the preparation of the membranes. However, electrodes with membranes based on NPOE had the best lifetime and response characteristics. The EMF measurements were carried out at zerocurrent conditions using a Lawson Lab 16 EMF station (multi-channel millivoltmeter) or a Metrohm 654 millivoltmeter. A double-junction reference Radelkis 0P0820P electrode with a $1 \mathrm{M} \mathrm{CH}_{3} \mathrm{COOLi}$ solution in the bridge cell was used. The measurements were carried out using cells of the type: $\mathrm{Ag} / \mathrm{AgCl}|1 \mathrm{M} \mathrm{KCl}| 1 \mathrm{M} \mathrm{CH}_{3} \mathrm{COOLi} \mid$ sample |

$$
\text { | membrane | }
$$

| 0.05 M MES/NaOH, $0.01 \mathrm{M} \mathrm{NaCl} \mid \mathrm{AgCl} / \mathrm{Ag}$.

At least three identical electrodes of the same membrane composition and containing the same inner electrolyte were prepared [39]. The studies were repeated several times over the period of one month.

To reduce the $\mathrm{pH}$ changes during the titrations solutions were prepared with $0.05 \mathrm{M}$ $\mathrm{MES} / \mathrm{NaOH}$ buffer of $\mathrm{pH}=5.5$ ((2-morpholino)ethanesulfonic acid monohydrate (MES)). All salt solutions contained $10^{-2} \mathrm{M} \mathrm{NaCl}$ as supporting electrolyte [22].

The selectivity coefficients $K_{A, B}^{\text {pot }}$ of the electrodes were determined by the separate solution method (SSM) and in some cases by the fixed interference method (FIM) $[40,41,42]$. The calibration curves were obtained by addition of standard solutions of different anions to $50 \mathrm{~cm}^{3}$ of $0.01 \mathrm{M} \mathrm{NaCl}$ in $0.05 \mathrm{M} \mathrm{MES} / \mathrm{NaOH}$ buffer solution of $\mathrm{pH}=5.5$. The concentration of the primary anion [A] was increased from $10^{-7}$ to $10^{-2} \mathrm{M}$. They were also measured by successive dilution of initial $5 \times 10^{-2} \mathrm{M}$ salt solutions until further dilution resulted in no potential change.

\section{Acknowledgements}

R.P. gratefully acknowledges the Gdansk University of Technology, Poland, and the Ministère de la Recherche et des Nouvelles Technologies, France (Cotutelles de thèses), for financial support. 


\section{References}

[1] Bianchi, A.; Bowman-James, K.; Garcia-Espana, E. Eds., Supramolecular Chemistry of Anions; John Wiley-VCH, New York, 1997, ch. 3, pp. 63.

[2] Kanyo, Z. F.; Christianson, D. W. J. Biol. Chem., 1991, 266, 4264.

[3] Pardee, A. P. J. Biol. Chem., 1966, 241, 5887.

[4] Puljak L.; Kilic, G. Biochim. Biophys. Acta, 2006, 1762, 404.

[5] Weiner, M. L.; Salminen, W. F.; Larson, P. R.; Barter, R. A.; Kranetz J. L.; Simon, G. S. Food and Chemical Toxicology, 2001, 39, 759.

[6] Matzer, W. E. Environmental Forensic, 2001, 2, 301.

[7] Bondy, C. R.; Gale P. A.; Loeb, S. J. J. Am. Chem. Soc., 2004, 126, 5030.

[8] Amendola, V.; Boiocchi, M.; Esteban-Gómez, D.; Fabbrizzi L.; Monzani, E. Org. Biomol. Chem., 2005, 3, 2632.

[9] Brooks, S. J.; Edwards, P. R.; Gale P. A.; Light, M. E. New J. Chem., 2006, 30, 65.

[10] Turner, D. R.; Paterson M. J.; Steed, J. W. J. Org. Chem., 2006, 71, 1598.

[11] Boerrigter, H.; Grave, L.; M. Nissink, J. W.; Chrisstoffels, L. A. J.; van der Maas, J. H.; Verboom, W.; de Jong F.; Reinhoudt, D. N.; J. Org. Chem., 1998, 63, 4174.

[12] Lee K. H.; Hong, J. I. Tetrahedron Lett., 2000, 41, 6083.

[13] ChmielewskiM.; Jurczak, J. Tetrahedron Lett., 2004, 45, 6007.

[14] Beer, P. D.; Drew M. G. B.; Gradwell, . K. J. Chem. Soc., Perkin Trans. 2, 2000, 511.

[15] Dietrich, B. Pure \& Appl. Chem., 1993, 65, 1457.

[16] Egorov, V. V.; Rakhman'ko, E. M.; Okaev E. B.; Pomelenok E. V.; Nazarov, V. A.; Talanta, 2004, 63, 119.

[17] Matthews S. E.; Beer, P. D. Calixarenes 2001; Asfari, Z.; Böhmer, V.; Harrowfield, J.; Vicens J. Eds., Kluwer Academic Publishers, Dordrecht, 2001, ch. 23, p. 421.

[18] Miao, R.; Zheng, Q. Y.; Chen C. F.; Huang Z. T. Tetrahedron Lett., 2004, 45, 4959. 
[19] Jagessar, R. C.; Shang, M.; Scheidt, W. R.; Burns; D. H. J. Am. Chem. Soc., 1998, 120, 11684.

[20] Gale, P. A.; Sessler, J. L.; Kral, V.; Lynch, V. J. Am. Chem. Soc., 1996, 118, 5140.

[21] Gale, P. A.; Sessler, J. L.; Allen, W. E.; Tvermoes, N. A.; Lynch, V. Chem. Commun., 1997, 665 .

[22] Pomecko, R.; Asfari, Z.; Hubscher-Bruder, V.; Bochenska M.; Arnaud-Neu, F. Supramol. Chem., 2007, 19, 459.

[23] Hamdi, A.; Nam, K. C.; Ryu, B.J.; Kim, J. S.; Vicens, J. Tetrahedron Letters 2004, 45, 4689.

[24] Broder, C. K.; Davidson, M. G.; Forsyth, V. T.; Howard, J. A. K.; Lamb S.; Mason, S. A. Crys. Grow. Des., 2002, 3, 163.

[25] Issleib K.; Muller, D.-W. Chem. Berichte, 1959, 92, 3175.

[26] Clark, P. W. Org. Prep. and Proc. Int., 1979, 11, 103.

[27] Mohr, W.; Horn, C. R.; Stahl J.; Gladysz, J. A. Synthesis, 2003, 8, 1279.

[28] Baklouti, L.; Cherif J.; Abidi, R.; Arnaud-Neu, F.; Harrowfield J.; Vicens, J. Org. Biomol. Chem., 2004, 2, 2786.

[29] Savage, P. B.; Holmgren S. K.; Gellman, S. H. J. Am. Chem. Soc., 1994, 116, 4069.

[30] Casabo, J.; Munoz J. A.; Errachid, A. Sens. Actuators, B, 1997, 43, 206.

[31] Shamsipur, M.; Soleymanpour, A.; Akhond, M.; Sharghi H.; Hasaninejad, A. R. Sens. Actuators, B, 2003, 89, 9.

[32] Dinten, O.; Spichiger, U. E.; Chaniotakis, N.; Gehrig, P.; Rusterholz, B.; Morf, W. E.; Simon, W. Anal. Chem., 1991, 63, 596.

[33] Lizondo-Sabater, J.; Segui, M. J.; Loris, J. M.; Martinez-Manez, R.; Pardo, T.; Sancenon, F.; Soto, J. Sens. Actuators B, 2004, 101, 20.

[34] Segui, M. J.; Lizondo-Sabater, J.; Martinez-Manez, R.; Soto, J. Analyst, 2002, 127, 387.

[35] Casabo, J.; Escriche, L.; Perez-Jimenez, C.; Munoz, J. A.; Teixidor, F.; Baussells, J.; Errachid, A. Anal. Chim. Acta, 1996, 320, 63.

[36] Schaller, U.; Bakker, E.; Spichiger, U. E.; Pretsch, E. Anal. Chem., 1994, 66, 391.

[37] Arnaud-Neu, F.; Delgado, R.; Chaves, S. Pure Appl. Chem., 2003, 75, 71.

[38] Hallen, D. Pure Appl. Chem., 1993, 65, 1527.

[39] Bochenska, M.; Zielenska, A.; Pomecko, R.; Hubscher-Bruder, V.; Arnaud-Neu, F. J. Incl. Phenom., 2005, 52, 129. 
1

2

3

4

5

6

7

8

9

10

11

12

13

14

15

16

17

18

19

20

21

22

23

24

25

26

27

28

29

30

31

32

33

34

35

36

37

38

39

40

41

42

43

44

45

46

47

48

49

50

51

52

53

54

55

56

57

58

59

60
[40] Buck, R. P.; Linder, E. Pure Appl. Chem., 1994, 66, 2527.

[41] Umezawa, Y.; Buhlmann, P.; Umezawa, K.; Tohda, K.; Amemiya, S. Pure Appl. Chem. 2000, 72, 1851.

[42] Lindner, E.; Umezawa, Y. Pure Appl. Chem. 2008, 80, 85. 
Table 1. Changes ( $\Delta \delta$ in ppm) in the ${ }^{31} \mathrm{P}$ NMR spectra of phosphonium ligands in the presence of sodium iodide, thiocyanate and perchlorate in $\mathrm{CDCl}_{3}$

\begin{tabular}{ccccc}
\hline & $\mathbf{1}$ & $\mathbf{2}$ & $\mathbf{5}$ & $\mathbf{6}$ \\
\hline Ligand $(\delta)$ & 25.797 & 25.962 & 25.756 & 25.982 \\
Ligand $+\mathrm{I}^{-}(\Delta \delta)$ & 0.215 & 0.203 & 0.210 & 0.175 \\
Ligand $+\mathrm{SCN}^{-}(\Delta \delta)$ & 0.470 & 0.530 & 0.767 & 0.965 \\
Ligand $+\mathrm{ClO}_{4}^{-}(\Delta \delta)$ & 0.602 & 0.620 & 0.992 & 1.170 \\
\hline
\end{tabular}

URL: http:/mc.manuscriptcentral.com/tandf/gsch Email: suprachem@mail.cm.utexas.edu 24 
Table 2. Differences $(\Delta \delta)$ in the proton chemical shifts $(\delta)[\mathrm{ppm}]$ in the spectra of ligands 5 and $\mathbf{6}, 5 \mathbf{a}$ and $\mathbf{6 a}, 5 \mathbf{b}$ and $\mathbf{6 b}$ in $\mathrm{CD}_{3} \mathrm{CN}$

\begin{tabular}{|c|c|c|c|c|c|c|}
\hline & C & e & $\mathbf{f}$ & g & $\overline{\mathbf{j}}$ & о \\
\hline$\delta(5)$ & 6.87 & 2.93 & 4.15 & 3.74 & 3.81 & - \\
\hline$\delta(5 a)$ & 6.83 & 2.91 & 4.11 & 3.76 & 3.17 & - \\
\hline$\Delta \delta(5 a-5)$ & -0.04 & -0.02 & -0.04 & 0.02 & -0.64 & - \\
\hline$\delta(5 \mathbf{b})$ & 6.80 & 2.87 & 4.09 & 3.77 & 3.06 & - \\
\hline$\Delta \delta(5 b-5)$ & -0.07 & -0.06 & -0.06 & 0.03 & -0.75 & - \\
\hline$\delta(6)$ & 6.92 & 3.02 & 4.19 & 3.70 & 3.58 & 2.78 \\
\hline$\delta(\mathbf{6 a})$ & 6.89 & 2.98 & 4.15 & 3.73 & 2.85 & 2.37 \\
\hline$\Delta \delta(6 a-6)$ & -0.03 & -0.04 & -0.04 & 0.03 & -0.73 & -0.41 \\
\hline$\delta(\mathbf{6 b})$ & 6.88 & 2.97 & 4.12 & 3.75 & 2.75 & 2.32 \\
\hline$\Delta \delta(6 \mathbf{b}-6)$ & -0.04 & -0.05 & -0.07 & 0.05 & -0.83 & -0.46 \\
\hline
\end{tabular}


Table 3. Characteristics of potentiometric responds for perchlorate of PVC/NPOE electrodes containing ligand $\mathbf{5}$ and $\mathbf{6}$ and different amount of KTClPB. (Inner and conditioning electrolyte $\mathrm{MES} / \mathrm{NaOH} \mathrm{pH}=5.5 / 10^{-2} \mathrm{M} \mathrm{NaCl}$ )

\begin{tabular}{cccc}
\hline Ligand & $\begin{array}{c}\text { KTClPB } \\
(\mathrm{mol} \%)\end{array}$ & $\begin{array}{c}\mathrm{S} \\
(\mathrm{mV} / \text { decade })\end{array}$ & $\begin{array}{c}\mathrm{LR} \\
(\log [\mathrm{A}])\end{array}$ \\
\hline $\mathbf{5}$ & 0 & -55.6 & -6.0 \\
$\mathbf{5}$ & 40 & -54.6 & -6.0 \\
$\mathbf{5}$ & 120 & -55.9 & -5.7 \\
$\mathbf{6}$ & 0 & -54.2 & -6.0 \\
$\mathbf{6}$ & 40 & -51.8 & -6.0 \\
$\mathbf{6}$ & 120 & -49.9 & -5.7 \\
\hline
\end{tabular}

URL: http:/mc.manuscriptcentral.com/tandf/gsch Email: suprachem@mail.cm.utexas.edu 26 
Table 4. Characteristics of potentiometric responds for perchlorate of PVC/NPOE electrodes containing tetrasubstituted phosphonium ligands with different counterions (Inner and conditioning electrolyte $\mathrm{MES} / \mathrm{NaOH} \mathrm{pH}=5.5 / 10^{-2} \mathrm{M}$ $\mathrm{NaCl})$.

\begin{tabular}{cccc}
\hline Ligand & Counterion & $\begin{array}{c}\mathrm{S} \\
(\mathrm{mV} / \text { decade })\end{array}$ & $\begin{array}{c}\mathrm{LR} \\
(\log [\mathrm{A}])\end{array}$ \\
\hline $\mathbf{5}$ & $\mathrm{Br}^{-}$ & -56.5 & -6.0 \\
$\mathbf{5 a}$ & $\mathrm{ClO}_{4}^{-}$ & -56.1 & -6.0 \\
$\mathbf{5 b}$ & $\mathrm{PF}_{6}^{-}$ & -38.3 & -5.5 \\
$\mathbf{6}$ & $\mathrm{Br}^{-}$ & -54.4 & -5.7 \\
$\mathbf{6 a}$ & $\mathrm{ClO}_{4}^{-}$ & -40.4 & -6.0 \\
$\mathbf{6 b}$ & $\mathrm{PF}_{6}^{-}$ & -36.2 & -6.0 \\
\hline
\end{tabular}


Table 5. Selectivity coefficients as $\log \boldsymbol{K}_{\mathrm{ClO}_{4}^{-}, \mathrm{X}}^{\text {pot }}$ of the PVC/NPOE membrane electrodes based on phosphonium calixarenes $\mathbf{1}$ - 6 and the monomer 8

\begin{tabular}{cccccccc}
\hline & \multicolumn{7}{c}{$\log \boldsymbol{K}_{\mathbf{C l O}_{4}^{-}, \boldsymbol{X}}^{\text {pot }}$} \\
Anion X & $\mathbf{1}$ & $\mathbf{2}$ & $\mathbf{3}$ & $\mathbf{4}$ & $\mathbf{5}$ & $\mathbf{6}$ & $\mathbf{8}$ \\
\cline { 2 - 8 } & 0 & 0 & 0 & 0 & 0 & 0 & 0 \\
$\mathrm{ClO}_{4}{ }^{-}$ & -1.2 & -1.4 & -1.1 & -0.6 & -1.3 & -1.4 & -1.1 \\
$\mathrm{SCN}^{-}$ & -1.7 & -1.7 & -1.3 & -0.9 & -2.0 & -2.0 & -1.6 \\
$\mathrm{I}^{-}$ & -2.6 & -2.6 & -2.4 & -1.9 & -2.9 & -3.0 & -2.5 \\
$\mathrm{NO}_{3}{ }^{-}$ & -4.6 & -4.5 & -4.6 & -4.4 & -4.6 & -4.4 & nd \\
$\mathrm{HCO}_{3}^{-}$ & -2.2 & -2.4 & -2.6 & -1.7 & -2.6 & -3.2 & -1.9 \\
$\mathrm{Cr}_{2} \mathrm{O}_{7}^{2-}$ & -4.5 & -4.5 & -4.5 & -4.4 & -4.5 & -4.4 & nd \\
$\mathrm{HPO}_{4}{ }^{2-}$ & -4.9 & -4.7 & -4.7 & -4.7 & -4.7 & -4.5 & nd \\
$\mathrm{SO}_{4}{ }^{2-}$ & & & & & & &
\end{tabular}

nd: not determined 


\section{Figure captions}

Figure 1. Chemical structures of the ligands under study

Figure 2. $\quad{ }^{1} \mathrm{H}$ NMR spectra of ligand 1 alone and in the presence of $\mathrm{ClO}_{4}{ }^{-}$in $\mathrm{CDCl}_{3}$

Figure 3. $\quad{ }^{1} \mathrm{H}$ NMR spectra of ligand 6 alone and in the presence of $\mathrm{ClO}_{4}{ }^{-}$in $\mathrm{CDCl}_{3}$

Figure 4. Potentiometric anion responses of electrodes with PVC/NPOE membrane containing ligand $\mathbf{1}$ in MES buffer at $\mathrm{pH}$ 5.5.

Figure 5. Potentiometric anion responses of electrodes with PVC/NPOE membrane containing ligand 6 in MES buffer at $\mathrm{pH}$ 5.5. 


\section{Supplementary Online Material}

\section{Anion recognition by phosphonium calix[4]arenes : synthesis and physico- chemical studies}

Radoslaw Pomecko, ${ }^{\mathrm{a}, \mathrm{b}}$ Zouhair Asfari, ${ }^{\mathrm{a}}$ Véronique Hubscher-Bruder, ${ }^{\mathrm{a}}$ Maria Bochenska ${ }^{\mathrm{b} *}$ and Françoise Arnaud-Neu ${ }^{\mathrm{a}^{*}}$

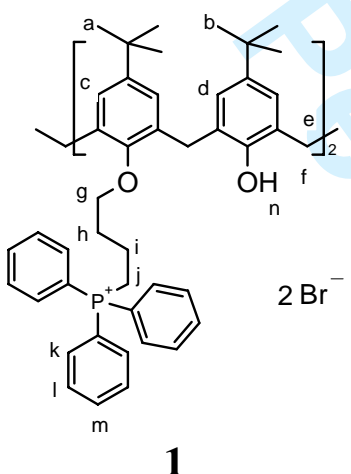

1

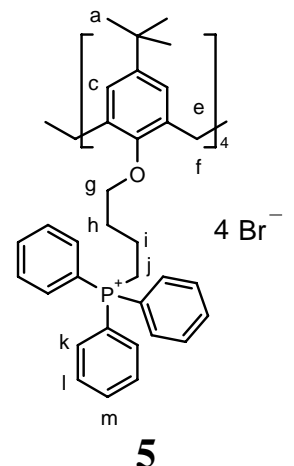

5
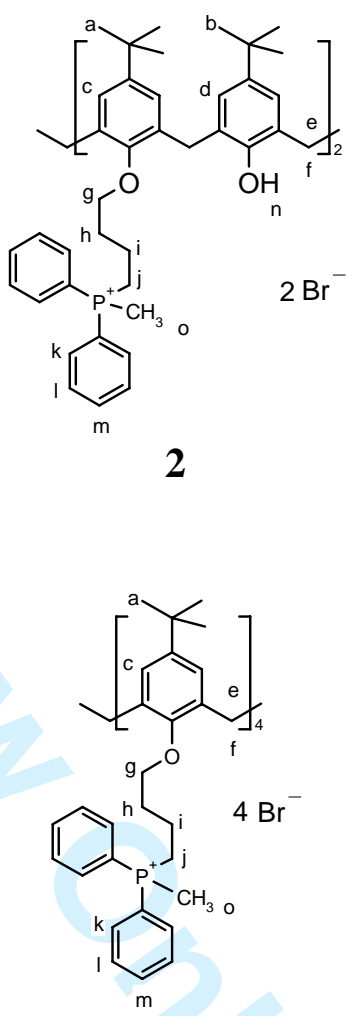

6 
Table S1. Changes $(\Delta \delta)$ of proton chemical shifts $(\delta)[\mathrm{ppm}]$ in the spectrum of ligand $\mathbf{1}$ in the presence of various sodium salts in $\mathrm{CDCl}_{3}$

\begin{tabular}{ccccccccccccccc}
\hline & $\mathbf{a}$ & $\mathbf{b}$ & $\mathbf{c}$ & $\mathbf{d}$ & $\mathbf{e}$ & $\mathbf{f}$ & $\mathbf{g}$ & $\mathbf{h}$ & $\mathbf{i}$ & $\mathbf{j}$ & $\mathbf{k}$ & $\mathbf{l}$ & $\mathbf{m}$ & $\mathbf{n}$ \\
\hline $\boldsymbol{\delta}(\mathbf{1})$ & 0.99 & 1.28 & 6.79 & 6.99 & 3.19 & 3.90 & 3.90 & 2.19 & 2.19 & 3.96 & 7.86 & 7.59 & 7.69 & 7.49 \\
$\boldsymbol{\delta}\left(\mathbf{1}+\mathbf{I}^{-}\right)$ & 0.99 & 1.28 & 6.79 & 6.99 & 3.19 & 3.91 & 3.90 & 2.19 & 2.19 & 3.85 & 7.84 & 7.60 & 7.71 & 7.49 \\
$\Delta \boldsymbol{\delta}$ & 0 & 0 & 0 & 0 & 0 & 0.01 & 0 & 0 & 0 & -0.11 & -0.02 & 0.01 & 0.02 & 0 \\
$\delta(\mathbf{1}+\mathbf{S C N})$ & 1.00 & 1.28 & 6.79 & 7.00 & 3.19 & 3.92 & 3.92 & 2.20 & 2.20 & 3.75 & 7.80 & 7.65 & 7.70 & 7.45 \\
$\Delta \boldsymbol{\delta}$ & 0.01 & 0 & 0 & 0.01 & 0 & 0.02 & 0.02 & 0.01 & -0.01 & -0.21 & -0.06 & 0.06 & 0.01 & -0.04 \\
$\boldsymbol{\delta}\left(\mathbf{1}+\mathbf{C l O}_{\mathbf{4}}\right)$ & 1.01 & 1.28 & 6.82 & 7.00 & 3.20 & 3.92 & 3.90 & 2.13 & 2.05 & 3.42 & 7.75 & 7.60 & 7.75 & 7.60 \\
$\Delta \boldsymbol{\delta}$ & 0.02 & 0 & 0.03 & 0.01 & 0.01 & 0.02 & 0 & -0.06 & -0.14 & -0.54 & -0.11 & -0.01 & 0.06 & -0.11 \\
\hline
\end{tabular}

Table S2. Changes $(\Delta \delta)$ of proton chemical shifts $(\delta)[\mathrm{ppm}]$ in the spectrum of ligand $\mathbf{2}$ in the presence of various sodium salts in $\mathrm{CDCl}_{3}$

\begin{tabular}{|c|c|c|c|c|c|c|c|c|c|c|c|c|c|c|c|}
\hline & $\mathbf{a}$ & b & C & d & e & $\mathbf{f}$ & g & $\mathbf{h}$ & i & $\mathbf{j}$ & $\mathbf{k}$ & 1 & $\mathbf{m}$ & $\mathbf{n}$ & $\mathbf{0}$ \\
\hline$\delta(2)$ & 0.96 & 1.29 & 6.76 & 7.01 & 3.23 & 4.02 & 3.90 & 2.18 & 2.00 & 3.67 & 7.98 & 7.54 & 7.65 & 7.31 & 2.99 \\
\hline$\delta\left(2+I^{-}\right)$ & 0.97 & 1.29 & 6.77 & 7.01 & 3.23 & 4.01 & 3.91 & 2.20 & 2.05 & 3.58 & 7.96 & 7.54 & 7.65 & 7.31 & 2.96 \\
\hline$\Delta \delta$ & 0.01 & 0 & 0.01 & 0 & 0 & -0.01 & 0.01 & 0.02 & 0.05 & -0.09 & -0.02 & 0 & 0 & 0 & -0.03 \\
\hline$\delta\left(2+\mathrm{SCN}^{-}\right)$ & 0.96 & 1.30 & 6.76 & 7.02 & 3.24 & 4.01 & 3.93 & 2.18 & 2.05 & 3.40 & 7.87 & 7.57 & 7.65 & 7.26 & 2.82 \\
\hline$\Delta \delta$ & 0 & 0.01 & 0 & 0.01 & 0.01 & -0.01 & 0.03 & 0 & 0.05 & -0.27 & -0.11 & 0.03 & 0 & -0.05 & -0.17 \\
\hline$\delta\left(2+\mathrm{ClO}_{4}^{-}\right)$ & 0.97 & 1.29 & 6.78 & 7.01 & 3.23 & 4.01 & 3.90 & 2.15 & 2.01 & 3.35 & 7.87 & 7.54 & 7.65 & 7.32 & 2.78 \\
\hline$\Delta \delta$ & 0.01 & 0 & 0.02 & 0 & 0 & -0.01 & 0 & -0.03 & 0.01 & -0.32 & -0.11 & 0 & 0 & 0.01 & -0.21 \\
\hline
\end{tabular}


Table S3. Changes $(\Delta \delta)$ of proton chemical shifts $(\delta)[\mathrm{ppm}]$ in the spectrum of ligand $\mathbf{6}$ in the presence of various sodium salts in $\mathrm{CDCl}_{3}$

\begin{tabular}{ccccccccccccc}
\hline & $\mathbf{a}$ & $\mathbf{c}$ & $\mathbf{e}$ & $\mathbf{f}$ & $\mathbf{g}$ & $\mathbf{h}$ & $\mathbf{i}$ & $\mathbf{j}$ & $\mathbf{k}$ & $\mathbf{l}$ & $\mathbf{m}$ & $\mathbf{0}$ \\
\hline $\boldsymbol{\delta}(\mathbf{6})$ & 1.03 & 6.71 & 3.03 & 4.26 & 3.79 & 2.39 & 1.69 & 3.79 & 8.10 & 7.66 & 7.66 & 2.91 \\
$\delta(\mathbf{6}+\mathbf{I})$ & 1.06 & 6.94 & 3.33 & 4.40 & 4.39 & 2.42 & 1.74 & 3.76 & 7.99 & 7.66 & 7.66 & 2.80 \\
$\Delta \boldsymbol{\delta}$ & 0.03 & 0.23 & 0.30 & 0.14 & 0.60 & 0.03 & 0.05 & -0.03 & -0.11 & 0 & 0 & -0.11 \\
$\boldsymbol{\delta}\left(\mathbf{6}+\mathbf{S C N}^{-}\right)$ & 1.06 & 6.94 & 3.30 & 4.37 & 4.28 & 2.40 & 1.79 & 3.50 & 7.88 & 7.69 & 7.69 & 2.73 \\
$\Delta \boldsymbol{\delta}$ & 0.03 & 0.23 & 0.27 & 0.11 & 0.49 & 0.01 & 0.10 & -0.29 & -0.22 & 0.03 & 0.03 & -0.18 \\
$\boldsymbol{\delta}\left(\mathbf{6}+\mathbf{C l O}_{\mathbf{4}}\right)$ & 1.07 & 6.95 & 3.33 & 4.38 & 4.27 & 2.33 & 1.66 & 3.20 & 7.82 & 7.63 & 7.63 & 2.50 \\
$\Delta \boldsymbol{\delta}$ & 0.04 & 0.24 & 0.30 & 0.12 & 0.48 & -0.06 & -0.03 & -0.59 & -0.30 & -0.03 & -0.03 & -0.41 \\
\hline
\end{tabular}


Table S4. Most important changes $(\Delta \delta)$ of protons chemical shifts $(\delta)[\mathrm{ppm}]$ in the spectra of ligands $\mathbf{5}$ and $\mathbf{5 b}$ in the presence of different perchlorate salts in $\mathrm{CD}_{3} \mathrm{CN}$

\begin{tabular}{|c|c|c|c|c|c|}
\hline 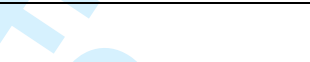 & C & e & $\mathbf{f}$ & g & $\mathbf{j}$ \\
\hline$\delta(5)$ & 6.87 & 2.93 & 4.15 & 3.74 & 3.81 \\
\hline$\delta\left(5+\mathrm{LiClO}_{4}\right)$ & 7.19 & 3.28 & 4.21 & 4.36 & 3.29 \\
\hline$\Delta \delta$ & 0.32 & 0.35 & 0.06 & 0.62 & -0.52 \\
\hline$\delta\left(5+\mathrm{NaClO}_{4}\right)$ & 7.22 & 3.33 & 4.21 & 4.09 & 3.28 \\
\hline$\Delta \delta$ & 0.35 & 0.40 & 0.06 & 0.35 & -0.53 \\
\hline$\delta\left(5+\mathrm{CsClO}_{4}\right)$ & 6.86 & 2.92 & 4.14 & 3.75 & 3.75 \\
\hline$\Delta \delta$ & -0.01 & -0.01 & -0.01 & 0.01 & -0.06 \\
\hline$\delta\left(5+\mathrm{NEt}_{4} \mathrm{ClO}_{4}\right)$ & 6.93 & 3.00 & 4.22 & 3.83 & 3.57 \\
\hline$\Delta \delta$ & 0.06 & 0.07 & 0.07 & 0.09 & -0.24 \\
\hline$\delta(5 b)$ & 6.80 & 2.87 & 4.09 & 3.77 & 3.06 \\
\hline$\delta\left(5 b+\mathrm{NaClO}_{4}\right)$ & 7.19 & 3.31 & 4.19 & 4.06 & 3.27 \\
\hline$\Delta \delta$ & 0.39 & 0.44 & 0.10 & 0.29 & 0.21 \\
\hline
\end{tabular}


Table S5. Thermodynamic parameters of the interaction between ligands $\mathbf{5}$ and $\mathbf{5 b}$ and several sodium and lithium salts.

\begin{tabular}{|c|c|c|c|c|c|}
\hline Ligand & Salt & Complexesl & $\log \beta$ & $-\Delta \mathrm{H}\left[\mathrm{kJ} \mathrm{mol}{ }^{-1}\right]$ & $\mathrm{T} \Delta \mathrm{S}\left[\mathrm{kJ} \mathrm{mol}{ }^{-1}\right]$ \\
\hline 5 & $\mathrm{NaClO}_{4}$ & $\begin{array}{c}\mathrm{ML} \\
\mathrm{ML}_{2}\end{array}$ & $\begin{array}{l}4.42 \\
7.95\end{array}$ & $\begin{array}{l}23.8 \\
30.7\end{array}$ & $\begin{array}{c}1.4 \\
14.6\end{array}$ \\
\hline $5 \mathbf{b}$ & $\mathrm{NaClO}_{4}$ & $\begin{array}{l}\mathrm{ML} \\
\mathrm{ML}_{2}\end{array}$ & $\begin{array}{l}3.14 \\
7.73\end{array}$ & $\begin{array}{l}51.7 \\
40.1\end{array}$ & $\begin{array}{c}-33.8 \\
4.0\end{array}$ \\
\hline 5 & $\mathrm{LiClO}_{4}$ & ML & 3.24 & 20.3 & -1.8 \\
\hline $5 \mathbf{b}$ & $\mathrm{LiClO}_{4}$ & $\begin{array}{c}\mathrm{ML} \\
\mathrm{ML}_{2}\end{array}$ & $\begin{array}{l}4.29 \\
7.34\end{array}$ & $\begin{array}{l}23.7 \\
35.0\end{array}$ & $\begin{array}{l}0.8 \\
6.8\end{array}$ \\
\hline 5 & $\mathrm{LiBr}$ & ML & 3.15 & 30.1 & -12.1 \\
\hline $5 \mathbf{b}$ & $\mathrm{LiBr}$ & $\begin{array}{l}\mathrm{ML} \\
\mathrm{ML}_{2}\end{array}$ & $\begin{array}{l}4.32 \\
7.91 \\
\end{array}$ & $\begin{array}{l}55.4 \\
72.7 \\
\end{array}$ & $\begin{array}{l}-30.8 \\
-27.6 \\
\end{array}$ \\
\hline
\end{tabular}


Table S6. Characteristics of anion response of PVC/NPOE membrane electrodes containing ligands 1 and 2 (Inner and conditioning electrolyte: $\mathrm{MES} / \mathrm{NaOH}, \mathrm{pH}=5.5 / 10^{-2} \mathrm{M} \mathrm{NaCl}$ )

\begin{tabular}{ccccccc}
\hline \multirow{2}{*}{$\begin{array}{c}\text { Primary } \\
\text { anion }\end{array}$} & $\begin{array}{c}\mathrm{DL} \\
(\log [\mathrm{A}])\end{array}$ & $\begin{array}{c}\mathrm{LR} \\
(\log [\mathrm{A}])\end{array}$ & $\begin{array}{c}\mathrm{S} \\
(\mathrm{mV} / \text { decade })\end{array}$ & $\begin{array}{c}\mathrm{DL} \\
(\log [\mathrm{A}])\end{array}$ & $\begin{array}{c}\mathrm{LR} \\
(\log [\mathrm{A}])\end{array}$ & $\begin{array}{c}\mathrm{S} \\
(\mathrm{mV} / \text { decade })\end{array}$ \\
\hline $\mathrm{ClO}_{4}{ }^{-}$ & -6.0 & -5.5 & -56 & -6.7 & -6.0 & -55 \\
$\mathrm{SCN}^{-}$ & -6.0 & -5.5 & -56 & -5.5 & -5.5 & -52 \\
$\mathrm{I}^{-}$ & -5.0 & -5.0 & -55 & -5.5 & -5.0 & -55 \\
$\mathrm{Br}^{-}$ & -4.5 & -4.2 & -31 & -4.0 & -3.5 & -47 \\
$\mathrm{NO}_{3}{ }^{-}$ & -4.4 & -4.0 & -51 & -4.0 & -3.6 & -39 \\
$\mathrm{HPO}_{4}{ }^{2-}$ & $\mathrm{nr}$ & $\mathrm{nr}$ & $\mathrm{nr}$ & $\mathrm{nr}$ & $\mathrm{nr}$ & $\mathrm{nr}$ \\
$\mathrm{Cr}_{2} \mathrm{O}_{7}{ }^{2-}$ & -4.8 & -4.3 & -43 & -4.8 & -4.2 & -43 \\
\hline $\mathrm{nr}-$ no response $^{-}$ & & & & &
\end{tabular}

Table S7. Characteristics of perchlorate response of PVC/NPOE membrane electrodes containing ligands $\mathbf{1}$ - 4 (Inner and conditioning electrolyte: $\mathrm{MES} / \mathrm{NaOH}, \mathrm{pH}=5.5 / 10^{-2} \mathrm{M} \mathrm{NaCl}$ )

\begin{tabular}{|c|c|c|c|}
\hline Ligand & $\begin{array}{c}\mathrm{DL} \\
(\log [\mathrm{A}])\end{array}$ & $\begin{array}{c}\mathrm{LR} \\
(\log [\mathrm{A}])\end{array}$ & $\begin{array}{c}\mathrm{S} \\
(\mathrm{mV} / \text { decade })\end{array}$ \\
\hline $\mathbf{1}$ & -6.0 & -5.5 & -56 \\
\hline $\mathbf{3}$ & -5.7 & -5.5 & -48 \\
\hline $\mathbf{2}$ & -6.7 & -6.0 & -55 \\
\hline $\mathbf{4}$ & -5.6 & -5.5 & -48 \\
\hline
\end{tabular}


Table S8. Characteristics of the electrodes containing ligands 5, 6 and 8

(Inner and conditioning electrolyte: $\mathrm{MES} / \mathrm{NaOH}, \mathrm{pH}=5.5 / 10^{-2} \mathrm{M} \mathrm{NaCl}$ )

\begin{tabular}{|c|c|c|c|c|c|c|c|c|c|}
\hline \multirow[b]{2}{*}{$\begin{array}{l}\text { Primary } \\
\text { anion }\end{array}$} & \multicolumn{3}{|c|}{5} & \multicolumn{3}{|c|}{6} & \multicolumn{3}{|c|}{8} \\
\hline & $\begin{array}{c}\mathrm{DL} \\
(\log [\mathrm{A}]) \\
\end{array}$ & $\begin{array}{c}\text { LR } \\
(\log [\mathrm{A}]) \\
\end{array}$ & $\begin{array}{c}\mathrm{S} \\
\text { (mV/ decade) } \\
\end{array}$ & $\begin{array}{c}\mathrm{DL} \\
(\log [\mathrm{A}])\end{array}$ & $\begin{array}{c}\text { LR } \\
(\log [\mathrm{A}])\end{array}$ & $\begin{array}{c}\mathrm{S} \\
\text { (mV/ decade) }\end{array}$ & $\begin{array}{c}\mathrm{DL} \\
(\log [\mathrm{A}])\end{array}$ & $\begin{array}{c}\mathrm{LR} \\
(\log [\mathrm{A}])\end{array}$ & $\begin{array}{c}\mathrm{S} \\
(\mathrm{mV} / \mathrm{dec} \text { de }) \\
\end{array}$ \\
\hline $\mathrm{ClO}_{4}^{-}$ & -6.6 & -6.4 & -56 & -6.0 & -6.2 & -53 & -5.6 & -5.4 & -51 \\
\hline $\mathrm{SCN}^{-}$ & -5.5 & -5.5 & -52 & -5.6 & -5.4 & -52 & -4.5 & -4.8 & -48 \\
\hline $\mathrm{I}^{-}$ & -5.5 & -5.2 & -55 & -5.5 & -5.2 & -52 & -4.5 & -4.8 & -48 \\
\hline $\mathrm{Br}^{-}$ & -3.8 & -3.8 & -44 & -3.8 & -3.8 & -11 & -2.6 & -3.0 & -10 \\
\hline $\mathrm{NO}_{3}^{-}$ & -5.0 & -4.5 & -51 & -4.5 & -4.5 & -25 & -3.4 & -4.0 & -22 \\
\hline $\mathrm{HCO}_{3}^{-}$ & -3.0 & -3.0 & -35 & -3.0 & -3.0 & -10 & -2.8 & -2.8 & -10 \\
\hline $\mathrm{Cr}_{2} \mathrm{O}_{7}{ }^{2-}$ & -4.0 & -5.5 & -31 & -5.0 & -5.5 & -46 & -4.0 & -4.6 & -43 \\
\hline
\end{tabular}



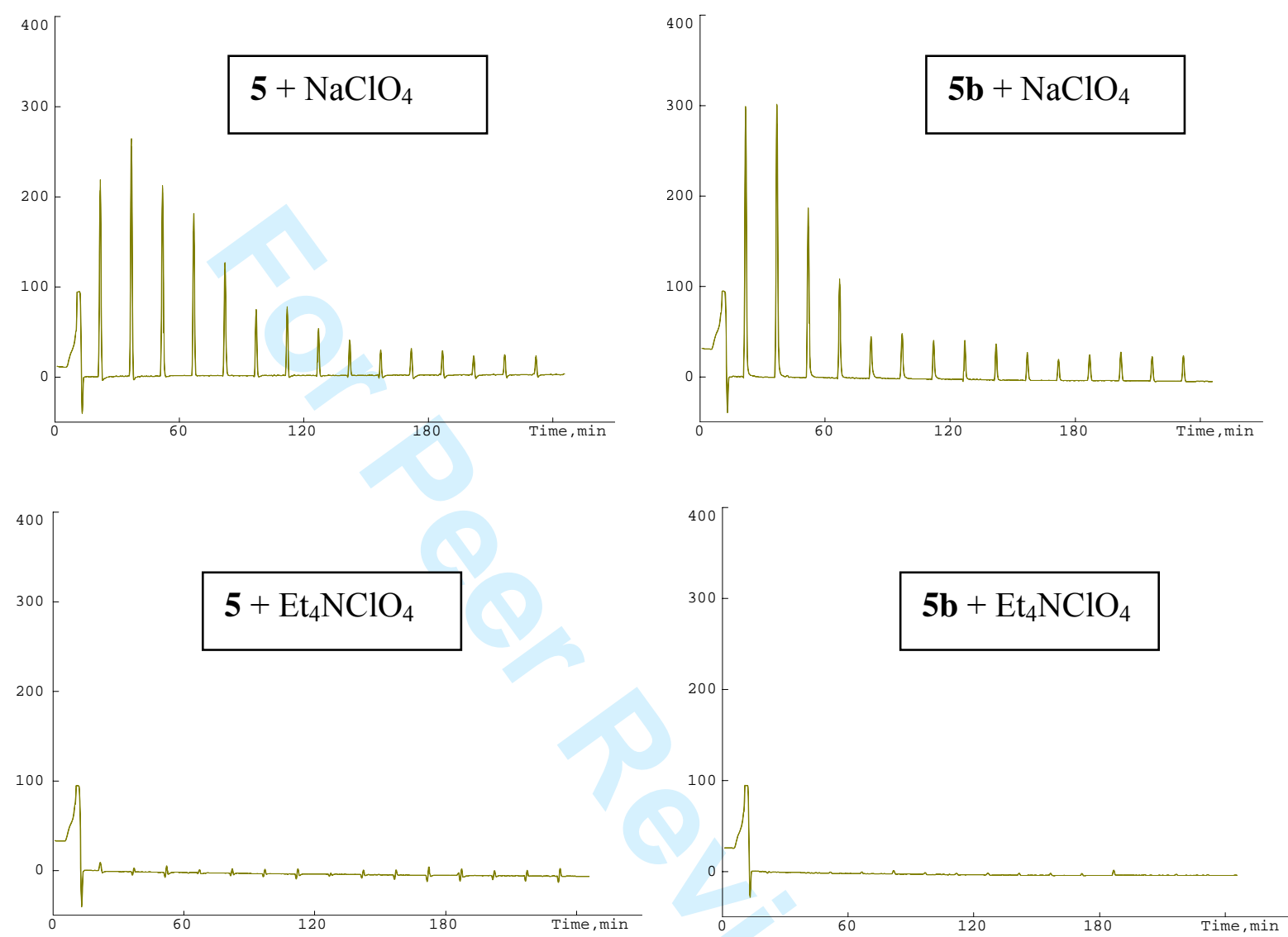

Figure S1. Thermograms corresponding to the titrations of ligands $\mathbf{5}$ and $\mathbf{5 b}$ against $\mathrm{NaClO}_{4}$ and $\mathrm{Et}_{4} \mathrm{NClO}_{4}$ in acetonitrile. 


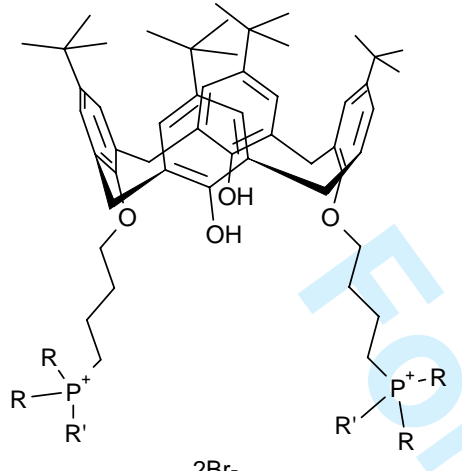

$2 \mathrm{Br}-$

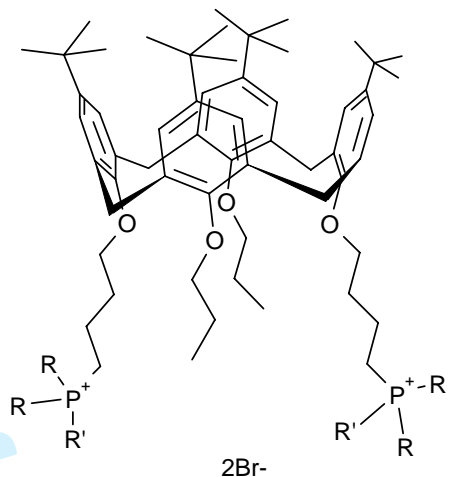

$2 \mathrm{Br}-$

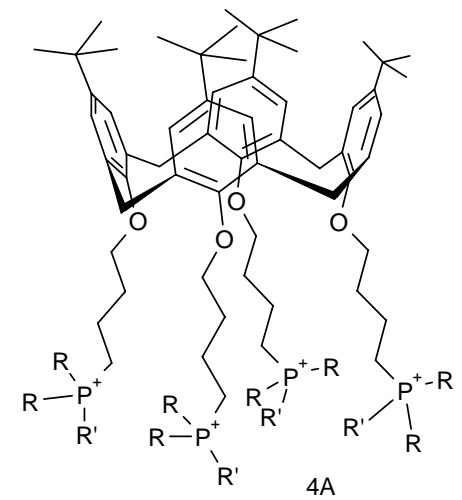

5: $\mathrm{R}, \mathrm{R}^{\prime}=\mathrm{Ph}, \mathrm{A}=\mathrm{Br}^{-}$

5a: $\mathrm{R}, \mathrm{R}^{\prime}=\mathrm{Ph}, \mathrm{A}=\mathrm{ClO}_{4}^{-}$

5b: $\mathrm{R}, \mathrm{R}^{\prime}=\mathrm{Ph}, \mathrm{A}=\mathrm{PF}_{6}^{-}$

6: $\mathrm{R}=\mathrm{Ph} \mathrm{R}^{\prime}=\mathrm{Me}, \mathrm{A}=\mathrm{Br}^{-}$

6a: $\mathrm{R}=\mathrm{Ph} \mathrm{R}=\mathrm{Me}, \mathrm{A}=\mathrm{ClO}_{4}^{-}$

6b: $\mathrm{R}=\mathrm{Ph} \mathrm{R}^{\prime}=\mathrm{Me}, \mathrm{A}=\mathrm{PF}_{6}^{-}$

7: $\mathrm{R}=\mathrm{Ph} \mathrm{R}^{\prime}=\mathrm{H}, \mathrm{A}=\mathrm{Br}^{-}$

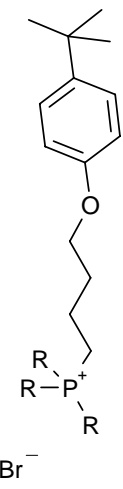

8: $\mathrm{R}=\mathrm{Ph}$
1: $\mathrm{R}, \mathrm{R}^{\prime}=\mathrm{Ph}$
2: $\mathrm{R}=\mathrm{Ph}, \mathrm{R}^{\prime}=\mathrm{Me}$
3: $\mathrm{R}, \mathrm{R}^{\prime}=\mathrm{Ph}$
4: $\mathrm{R}=\mathrm{Ph}, \mathrm{R}^{\prime}=\mathrm{Me}$ 

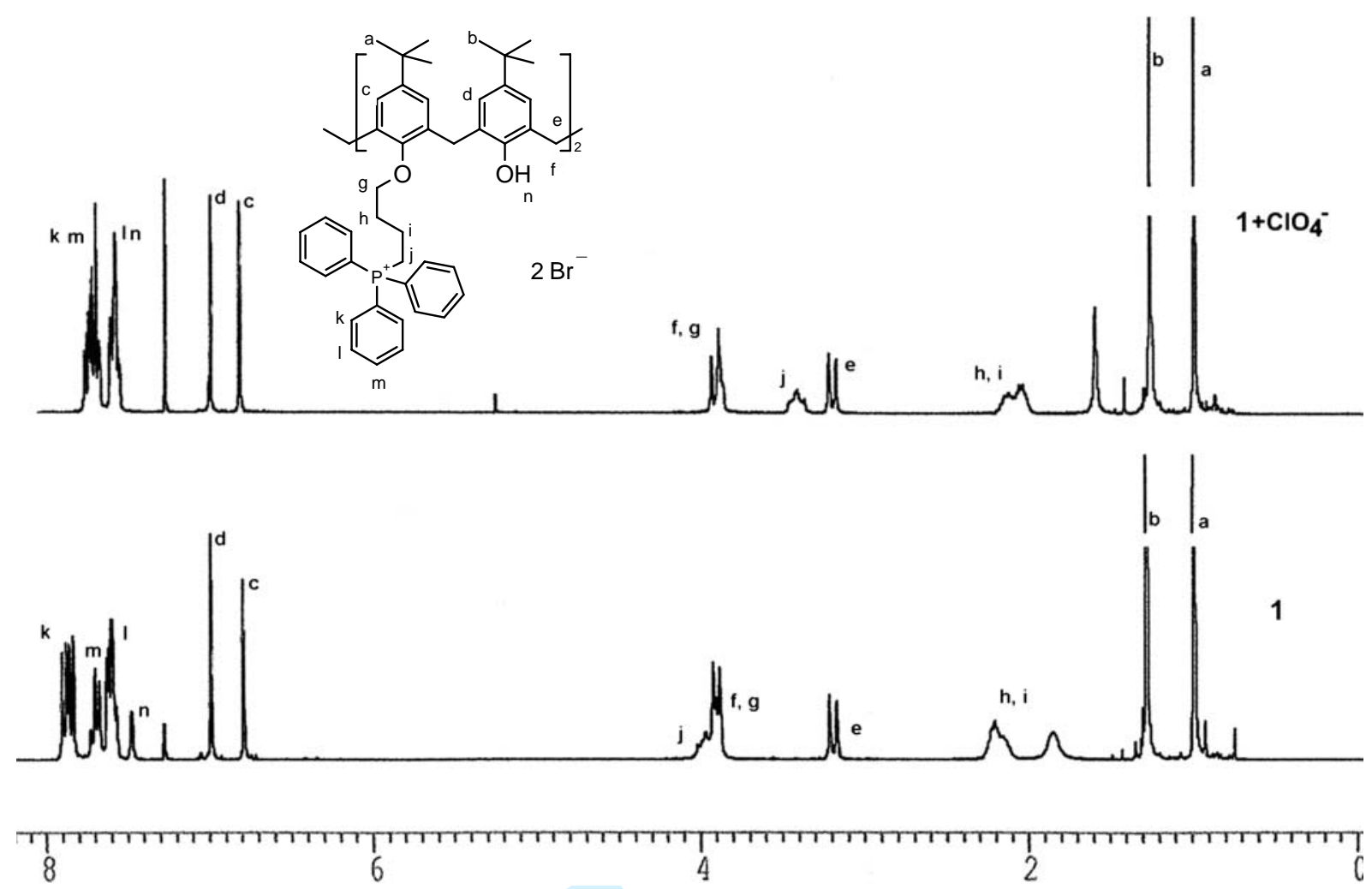


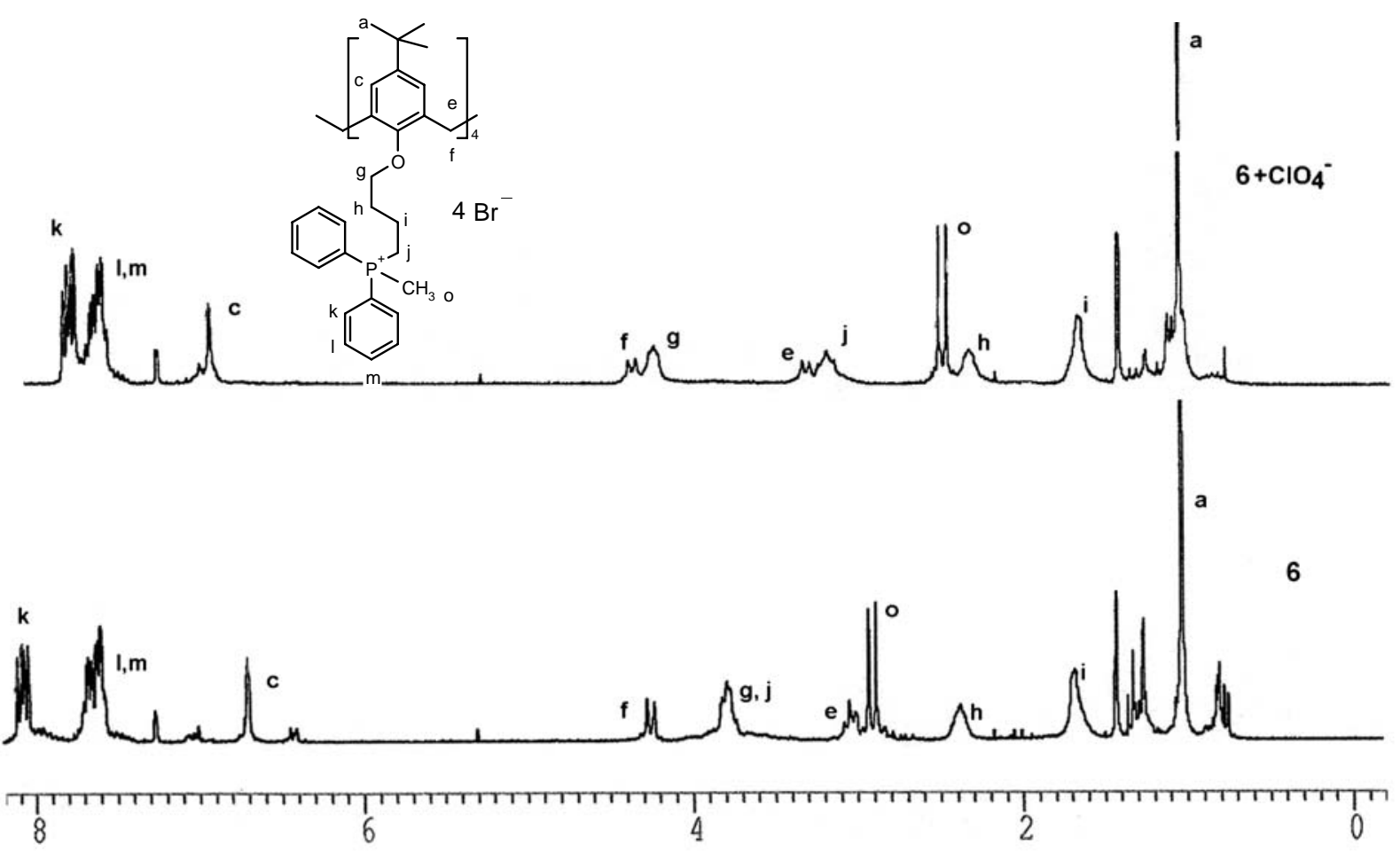




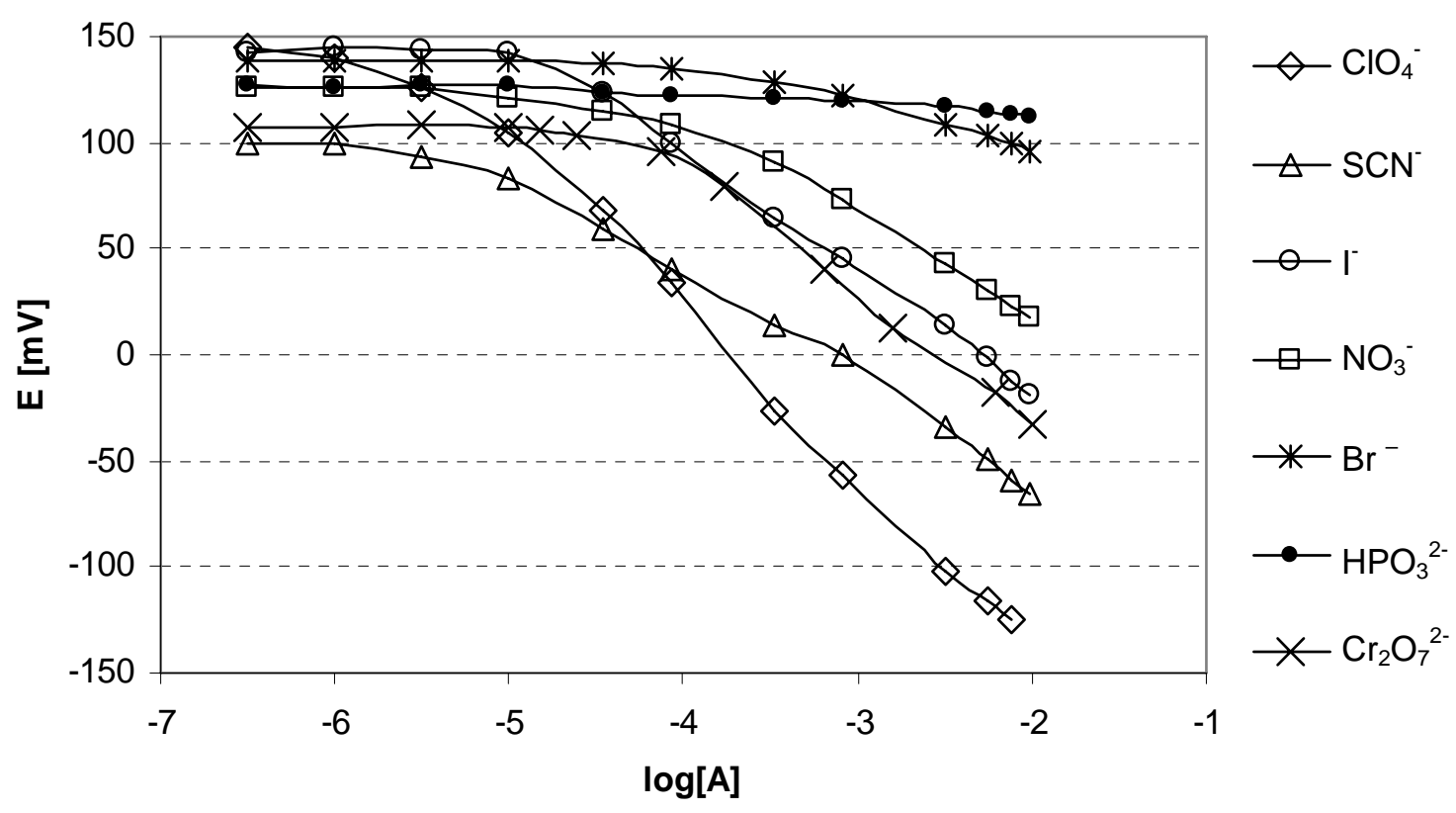




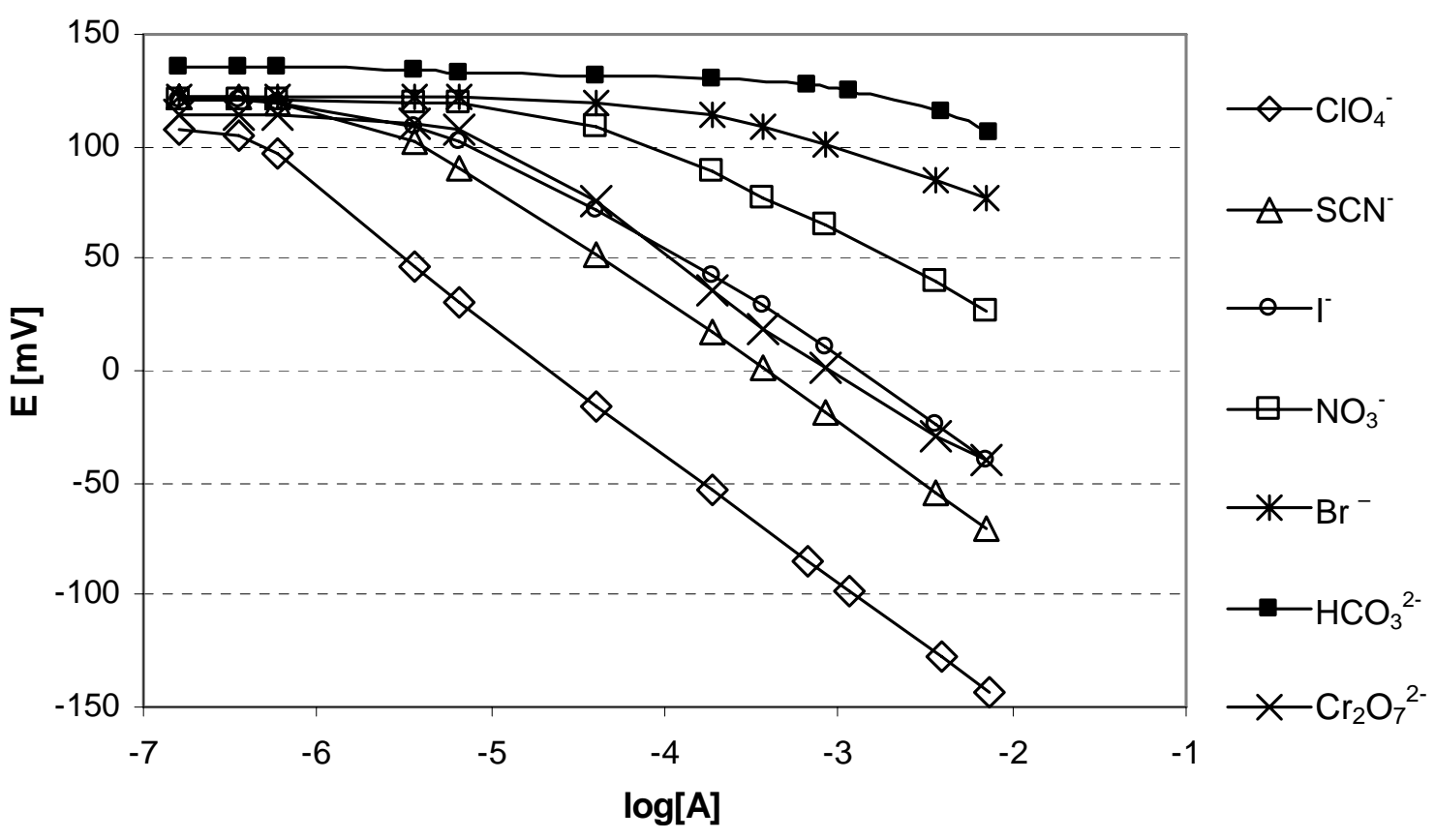


Scheme 1

$169 \times 135 \mathrm{~mm}(600 \times 600 \mathrm{DPI})$ 


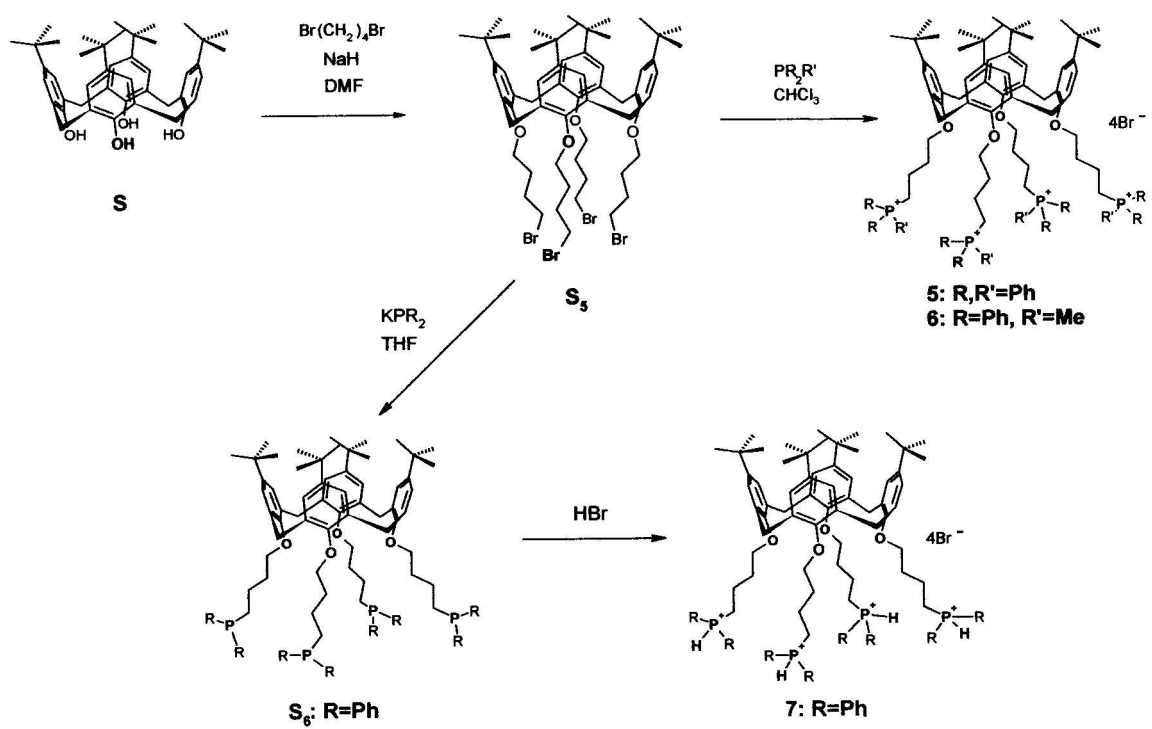

Scheme 2

Scheme 2

$171 \times 138 \mathrm{~mm}(600 \times 600 \mathrm{DPI})$ 